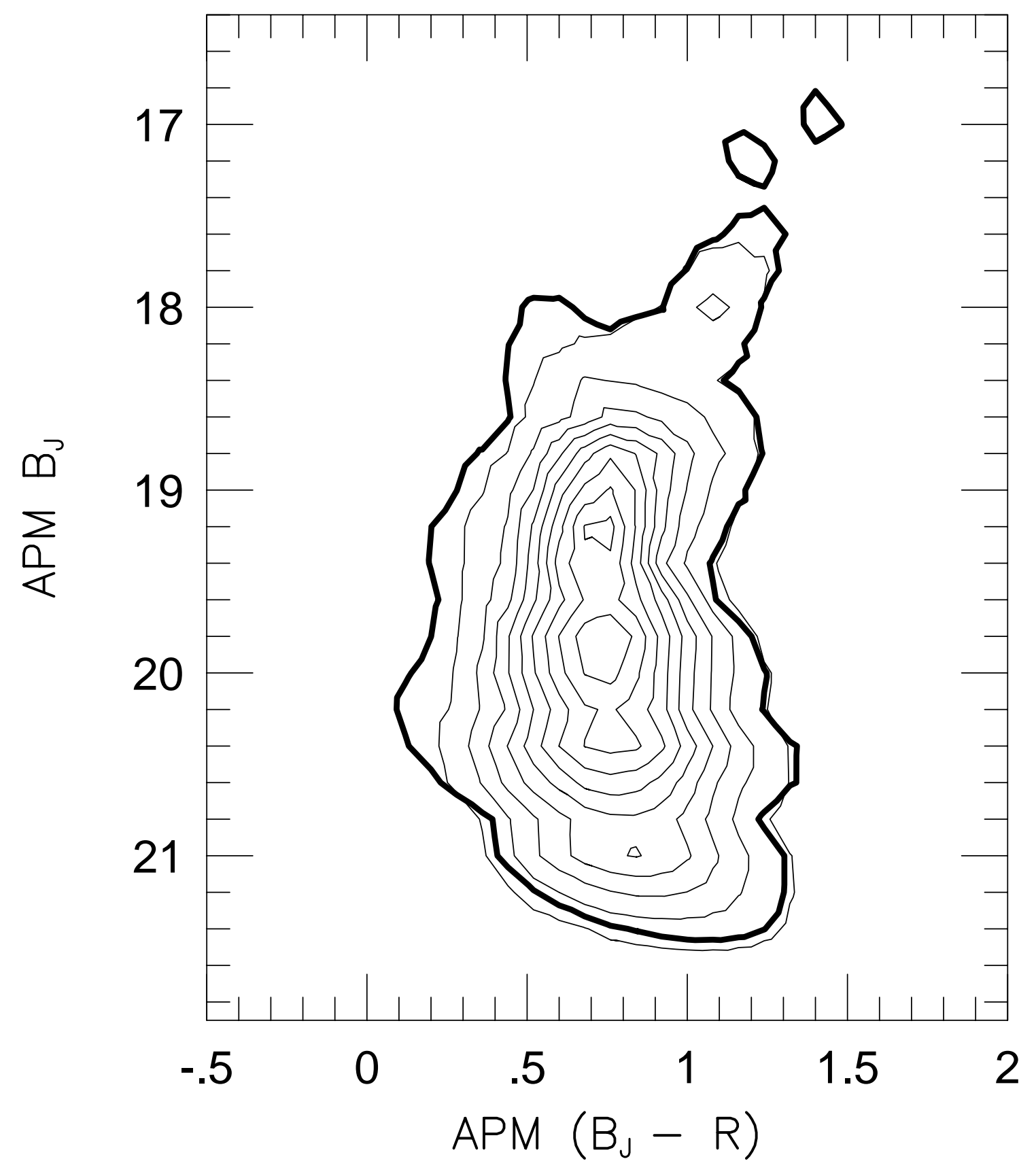




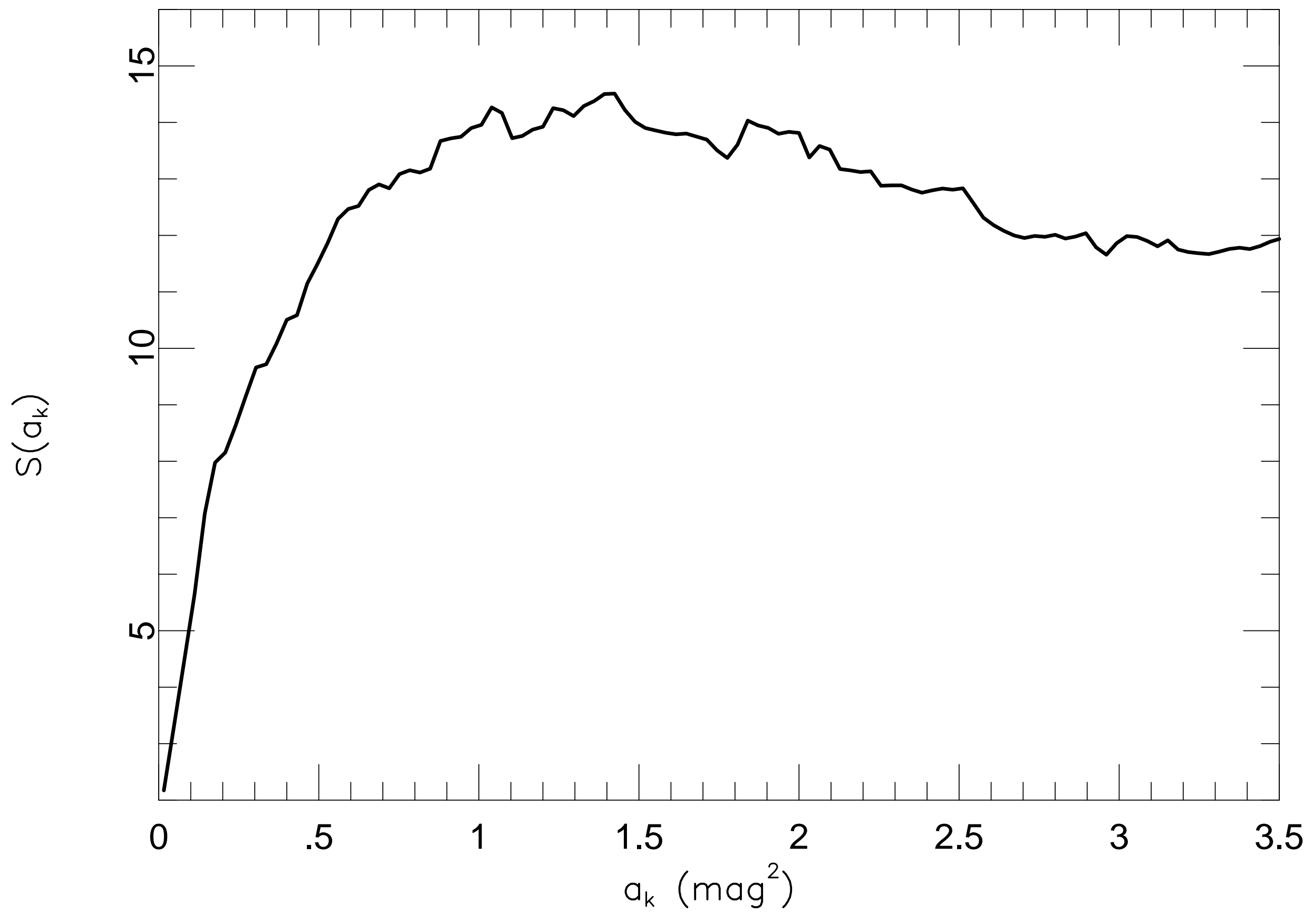




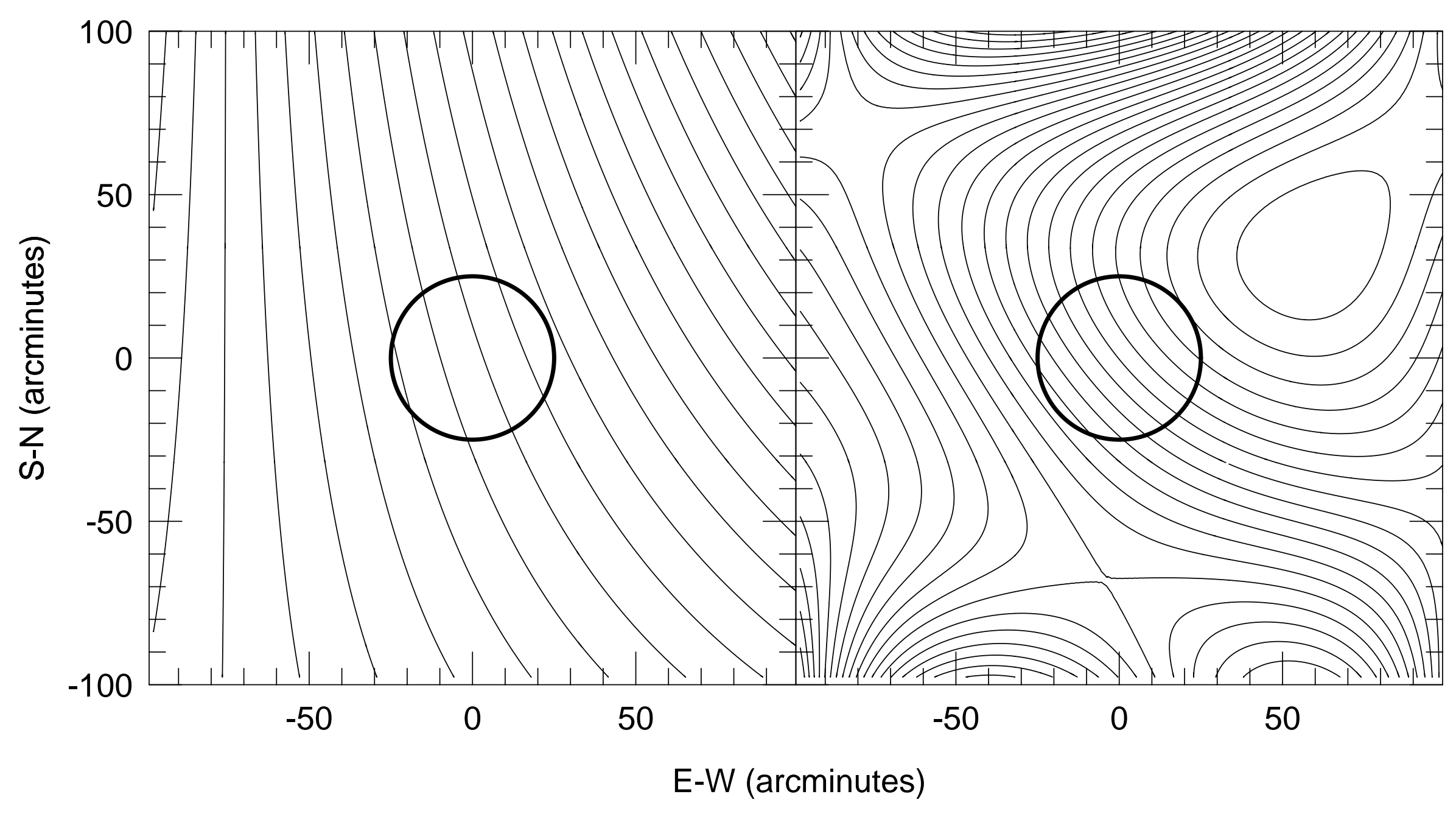




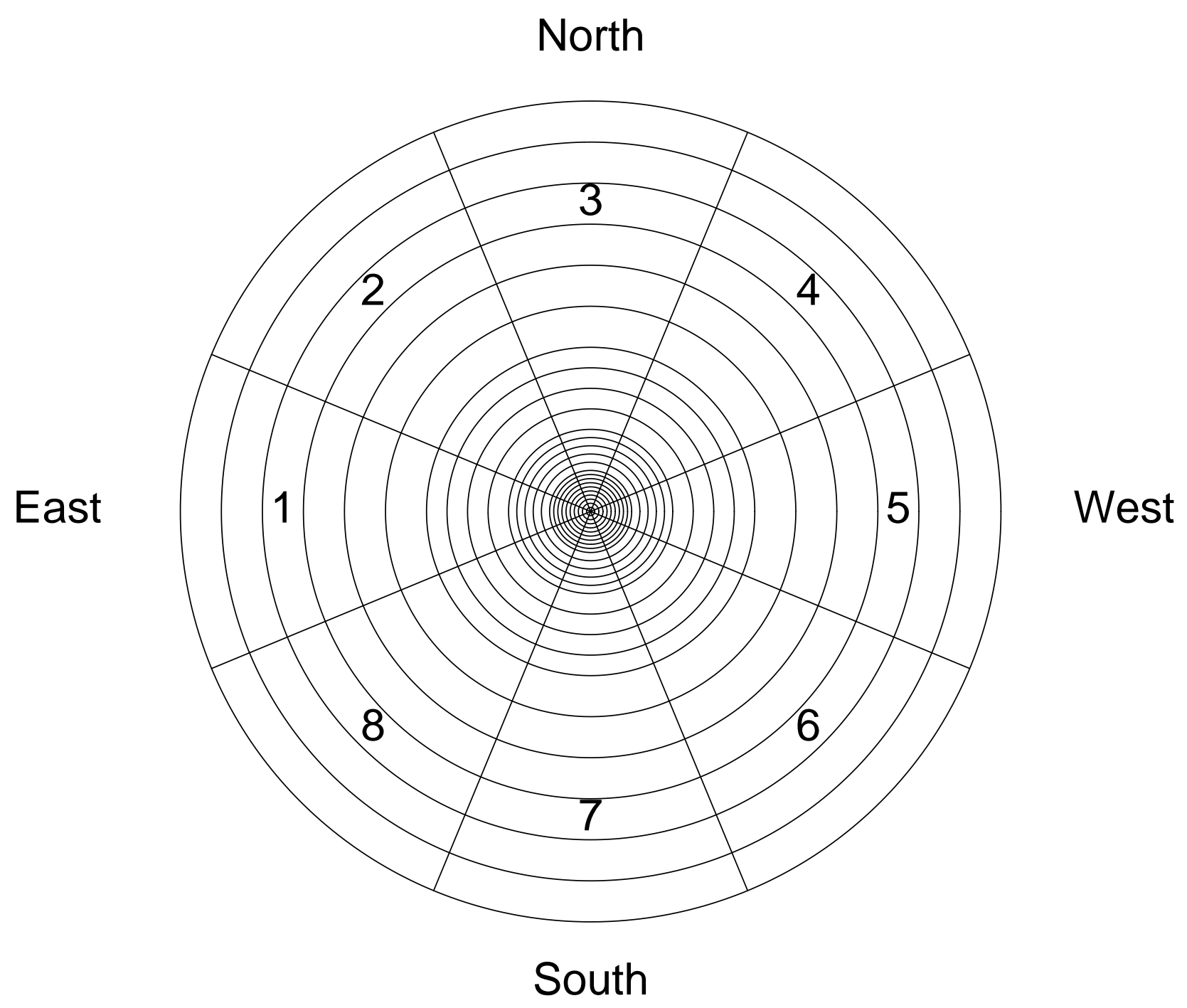




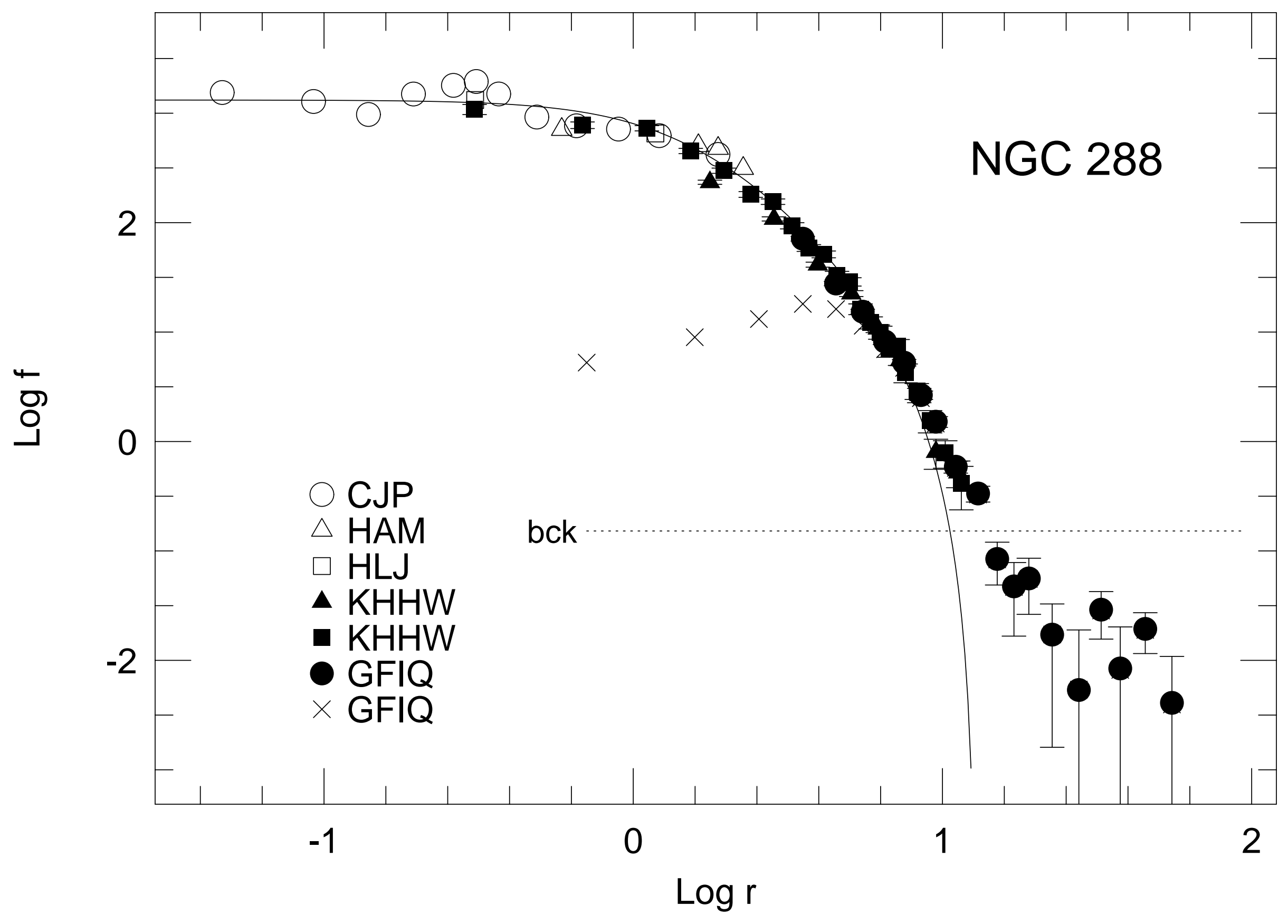




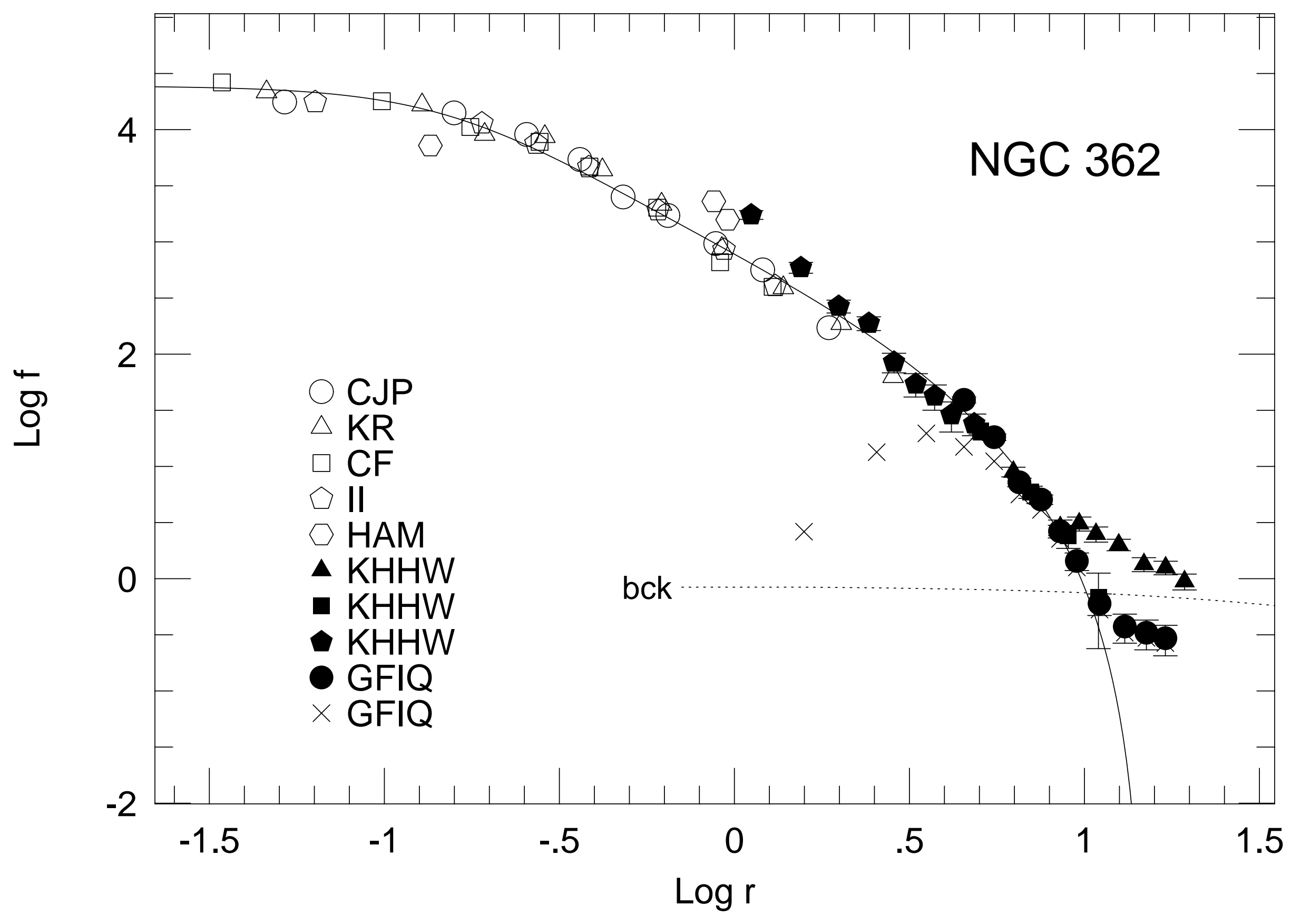




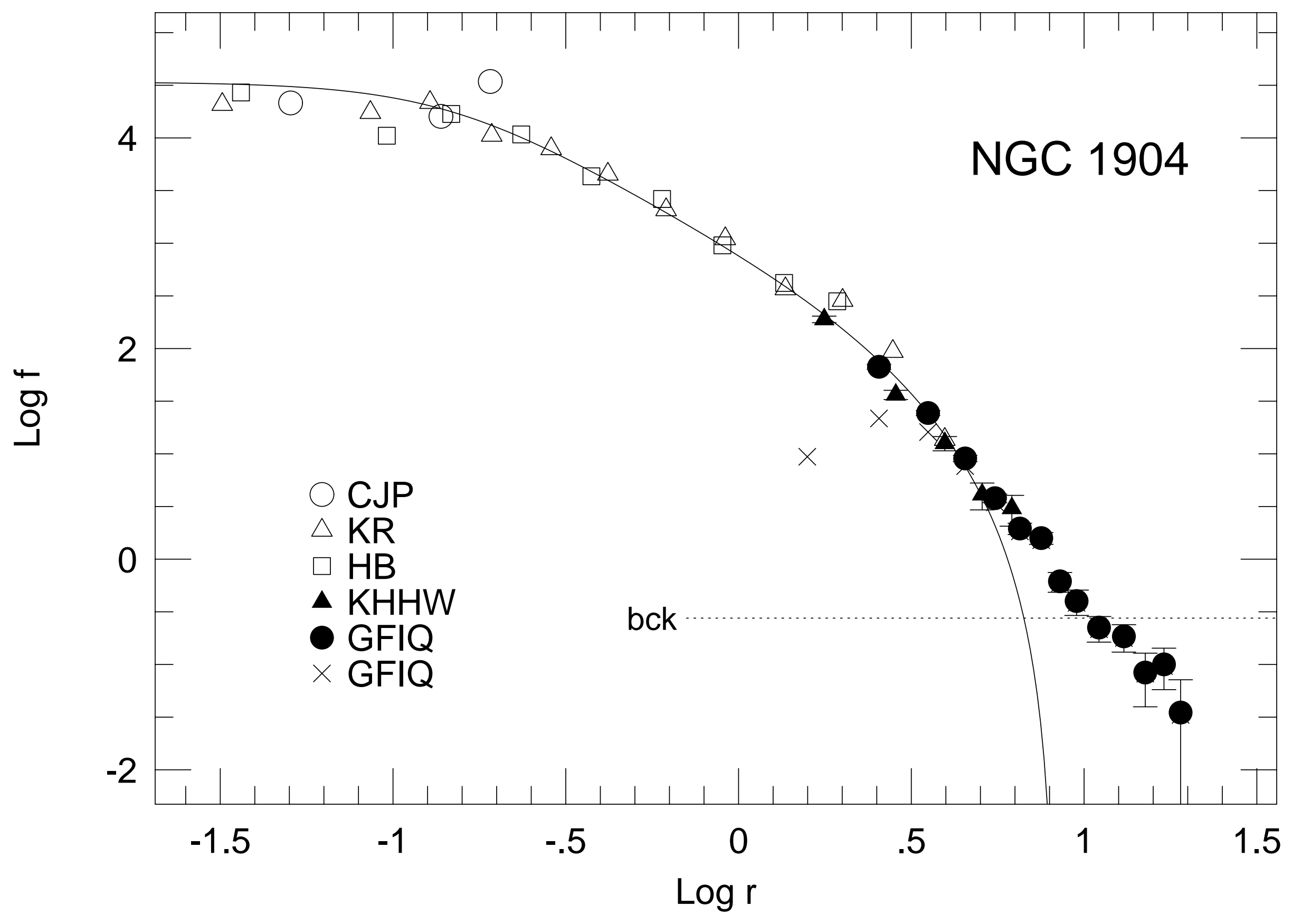




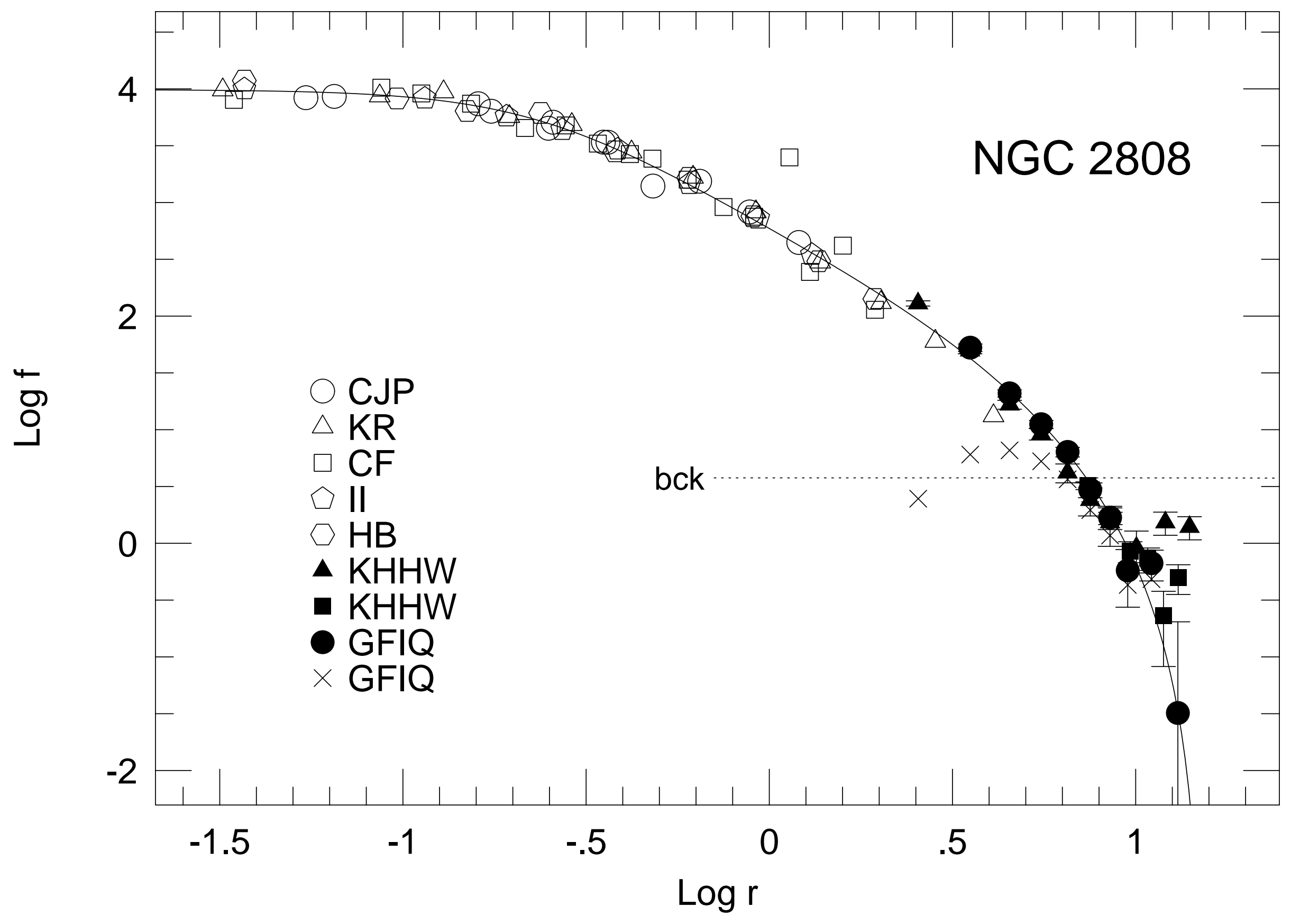




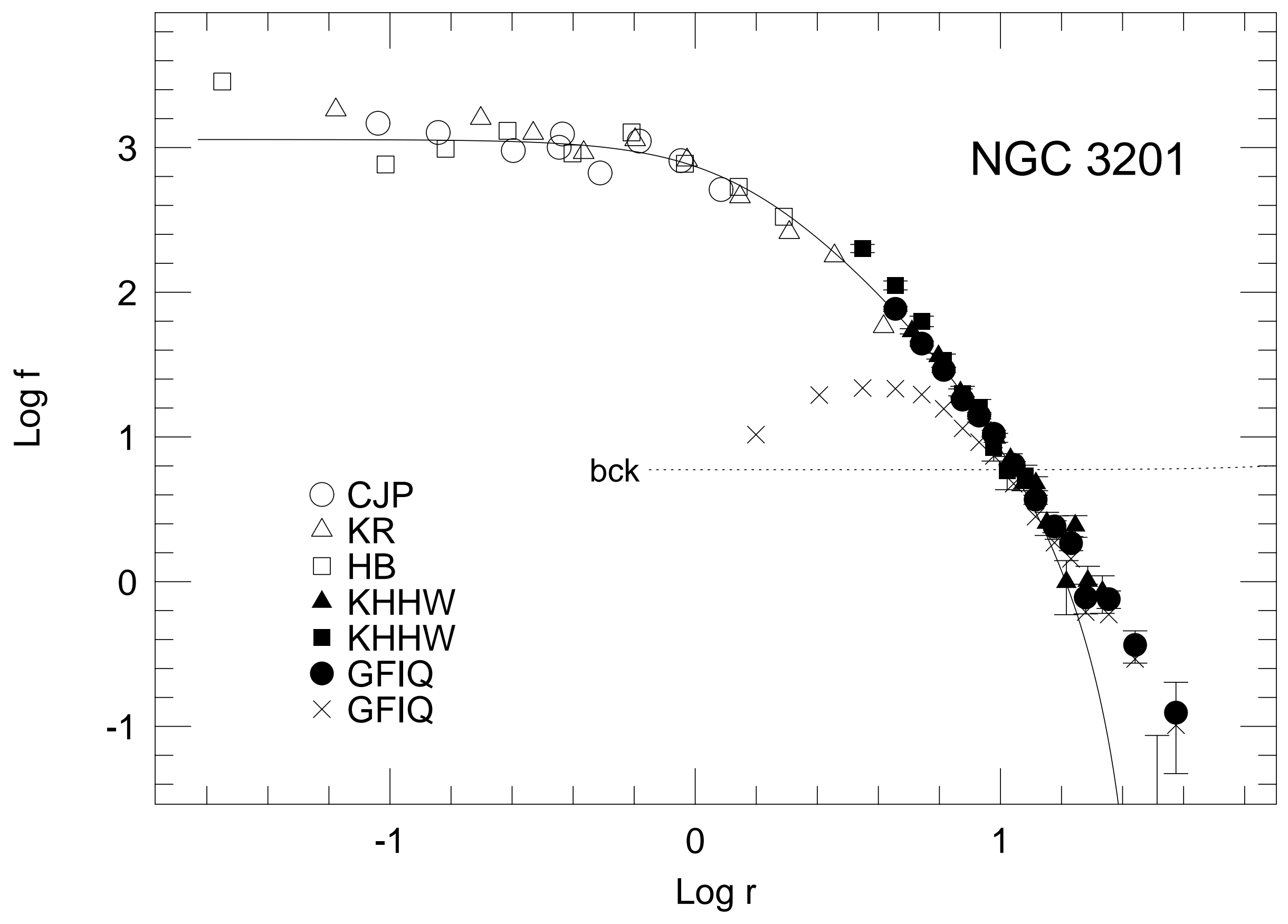




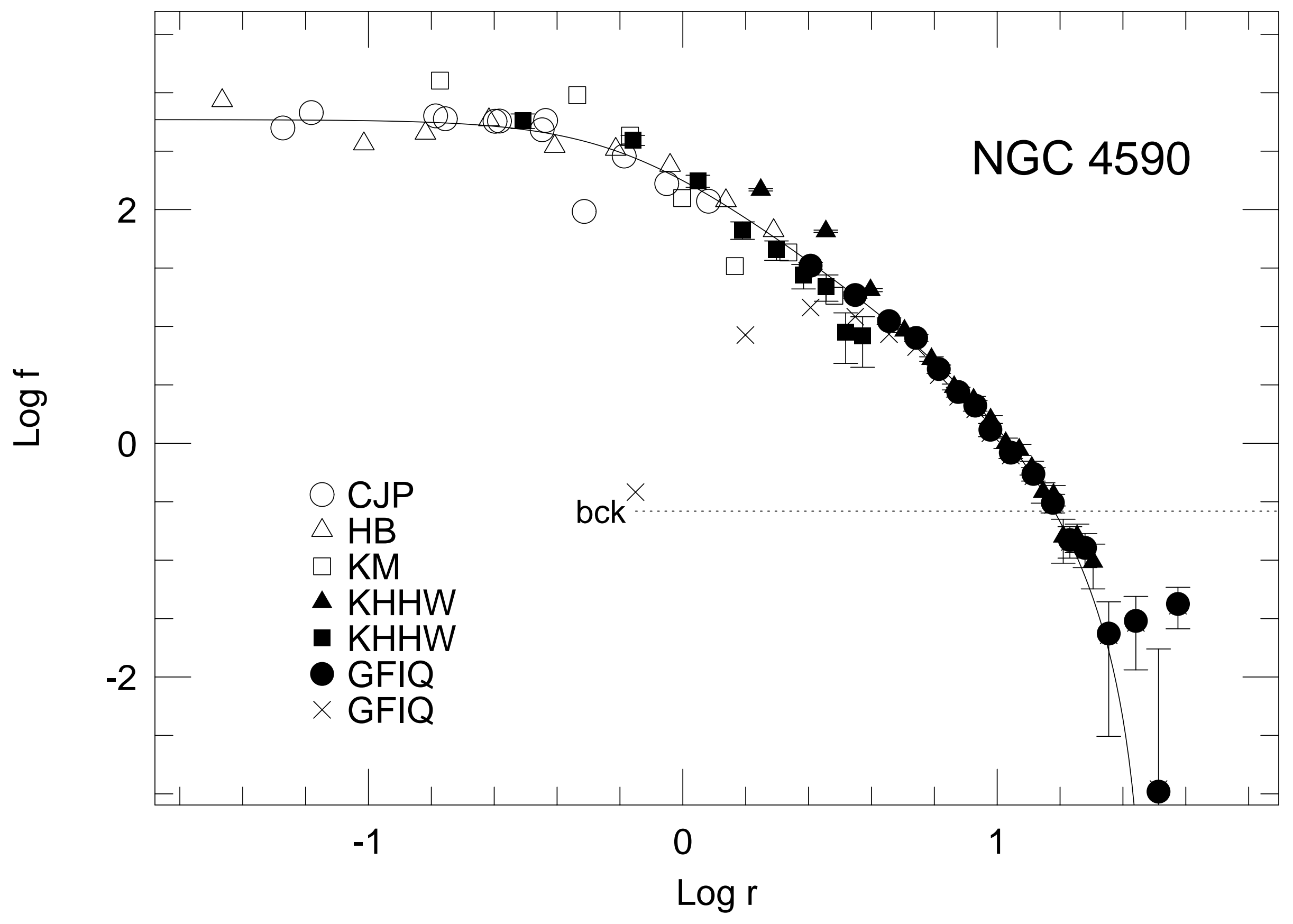




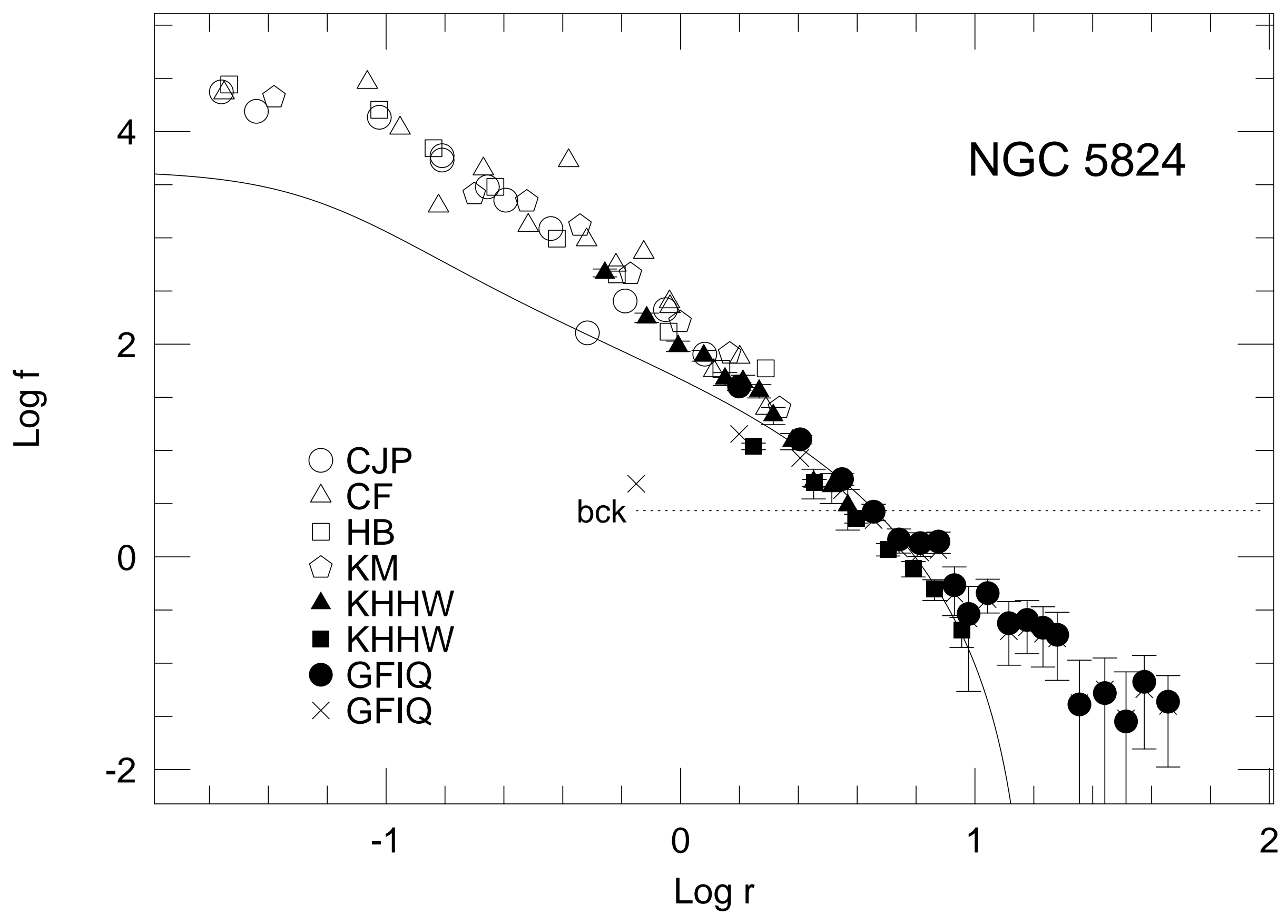




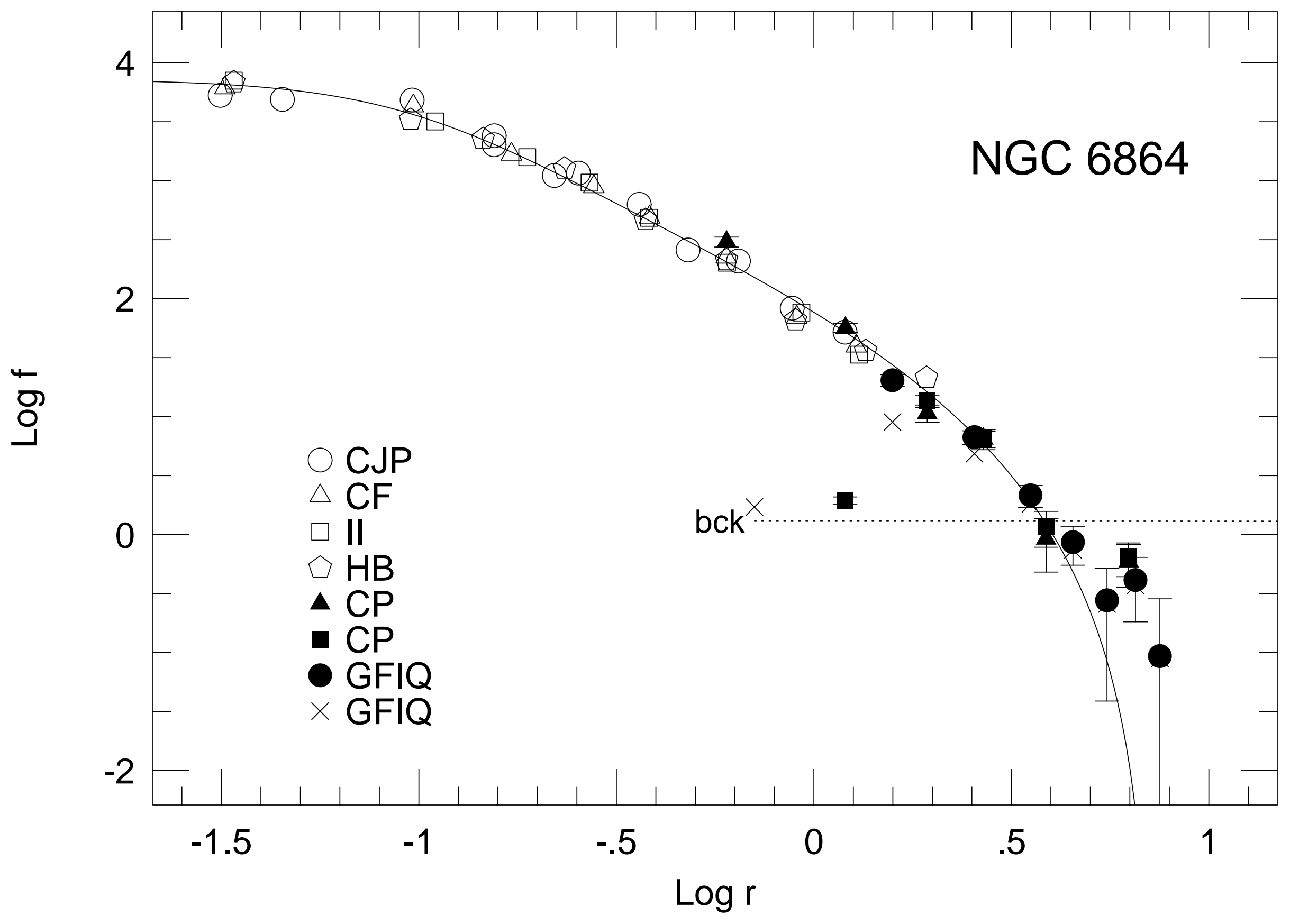




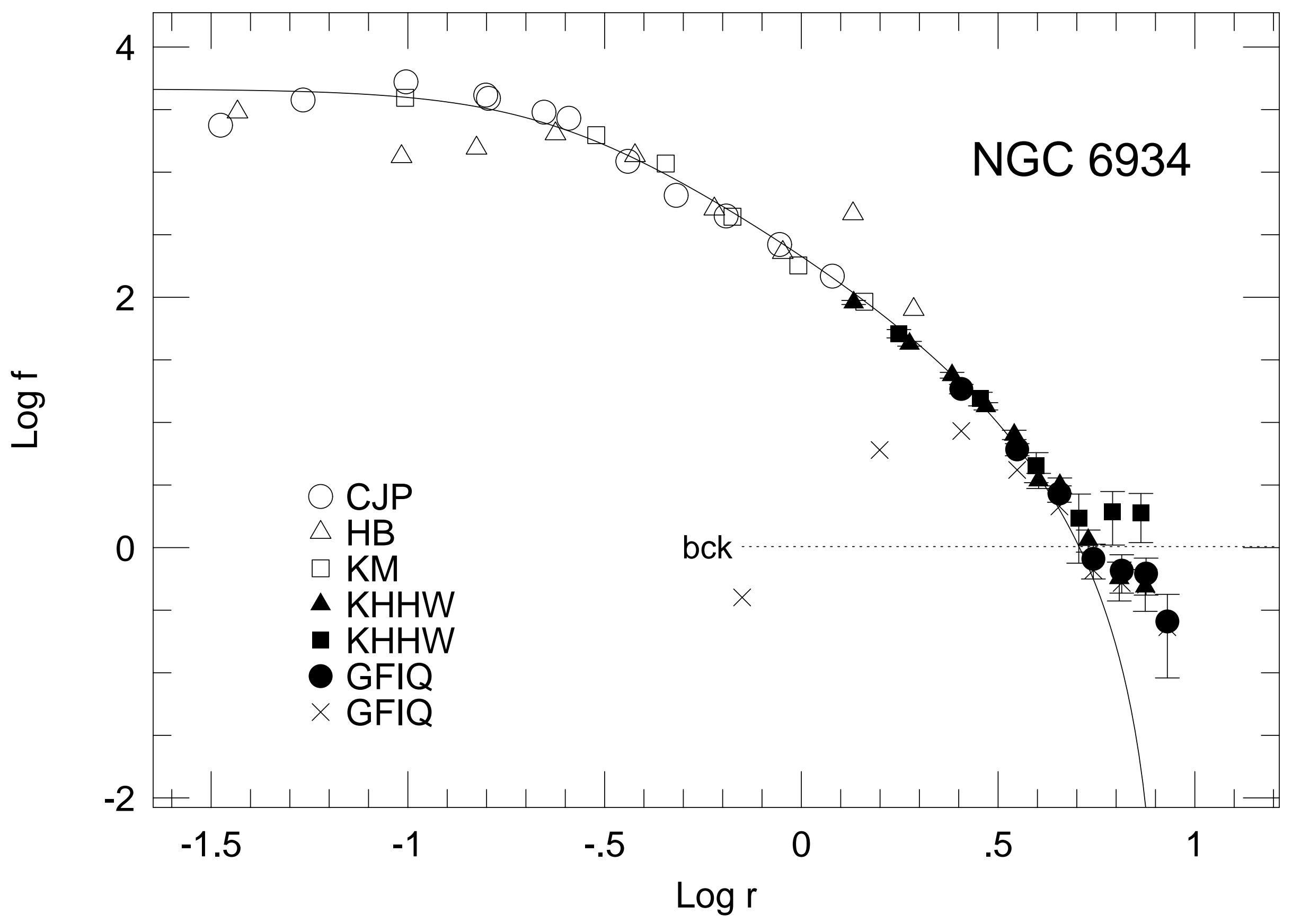




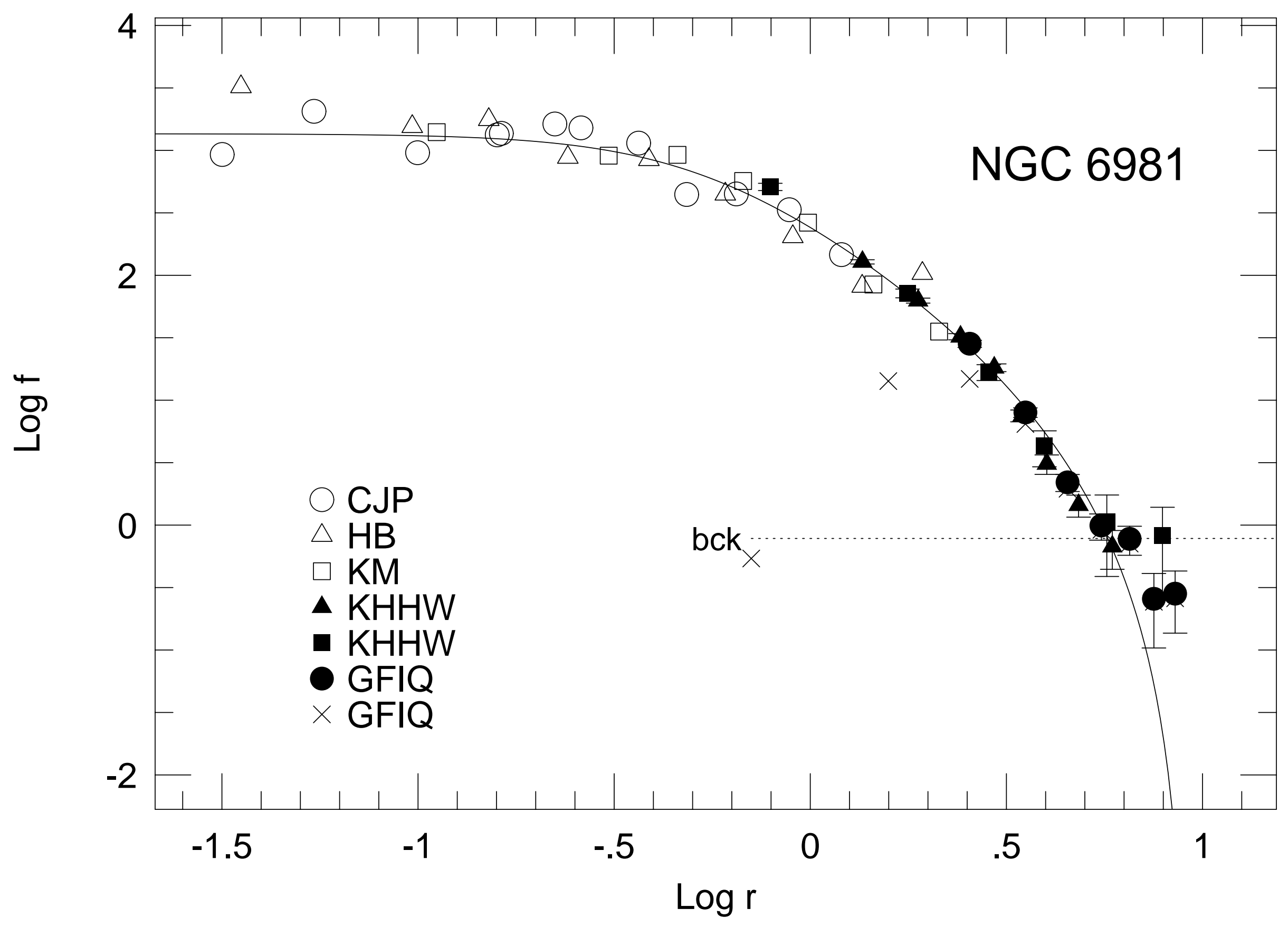




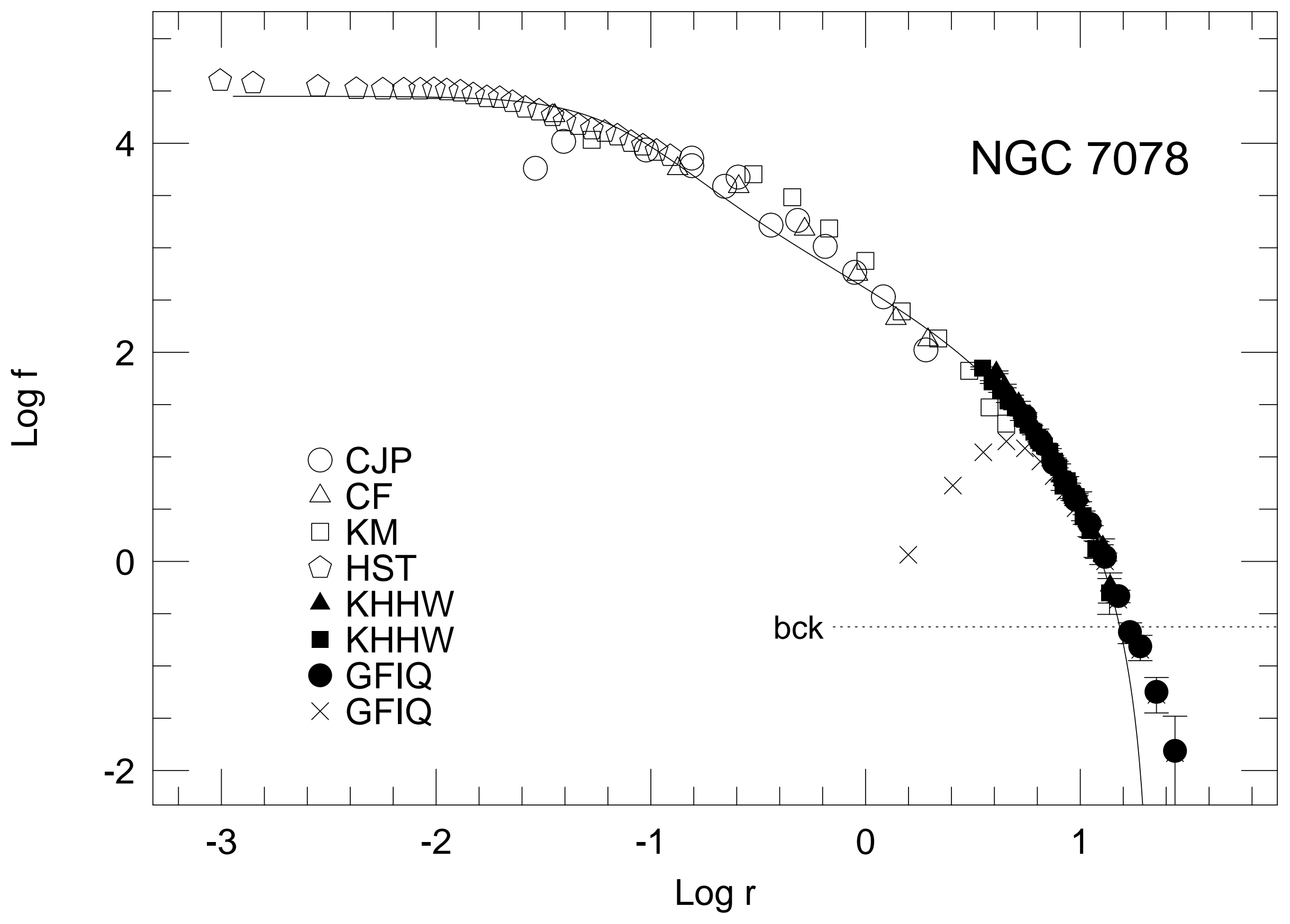




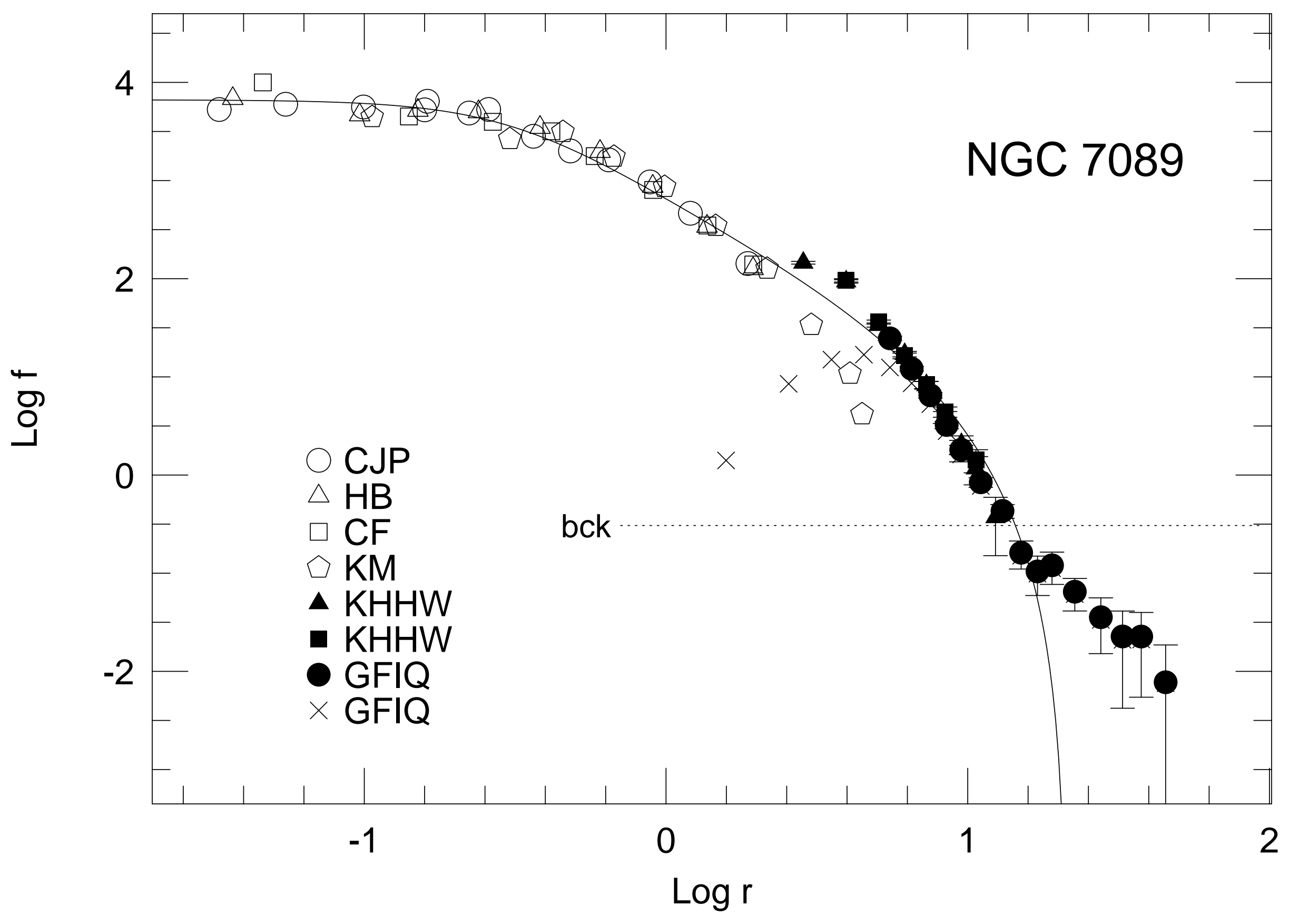




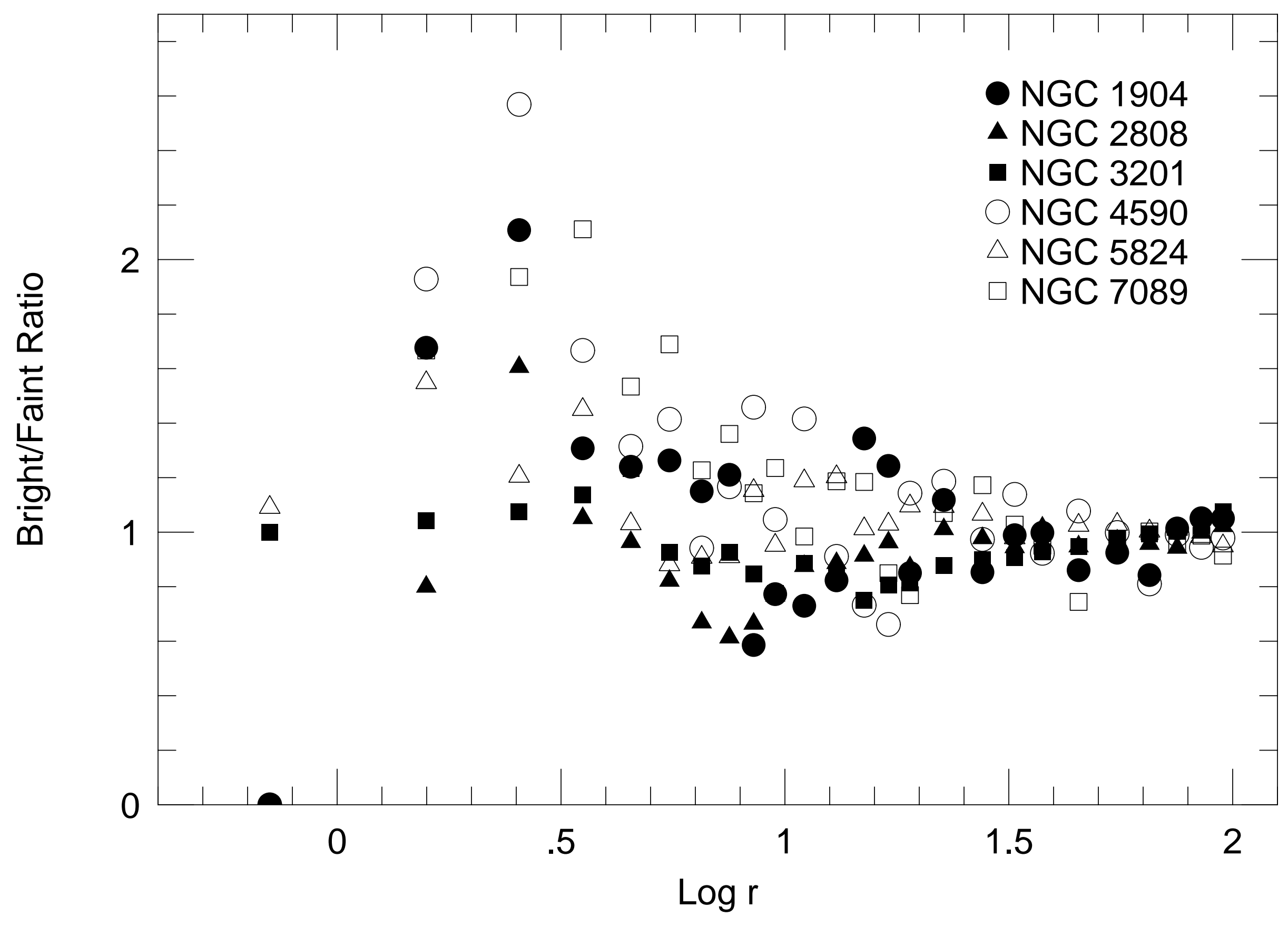




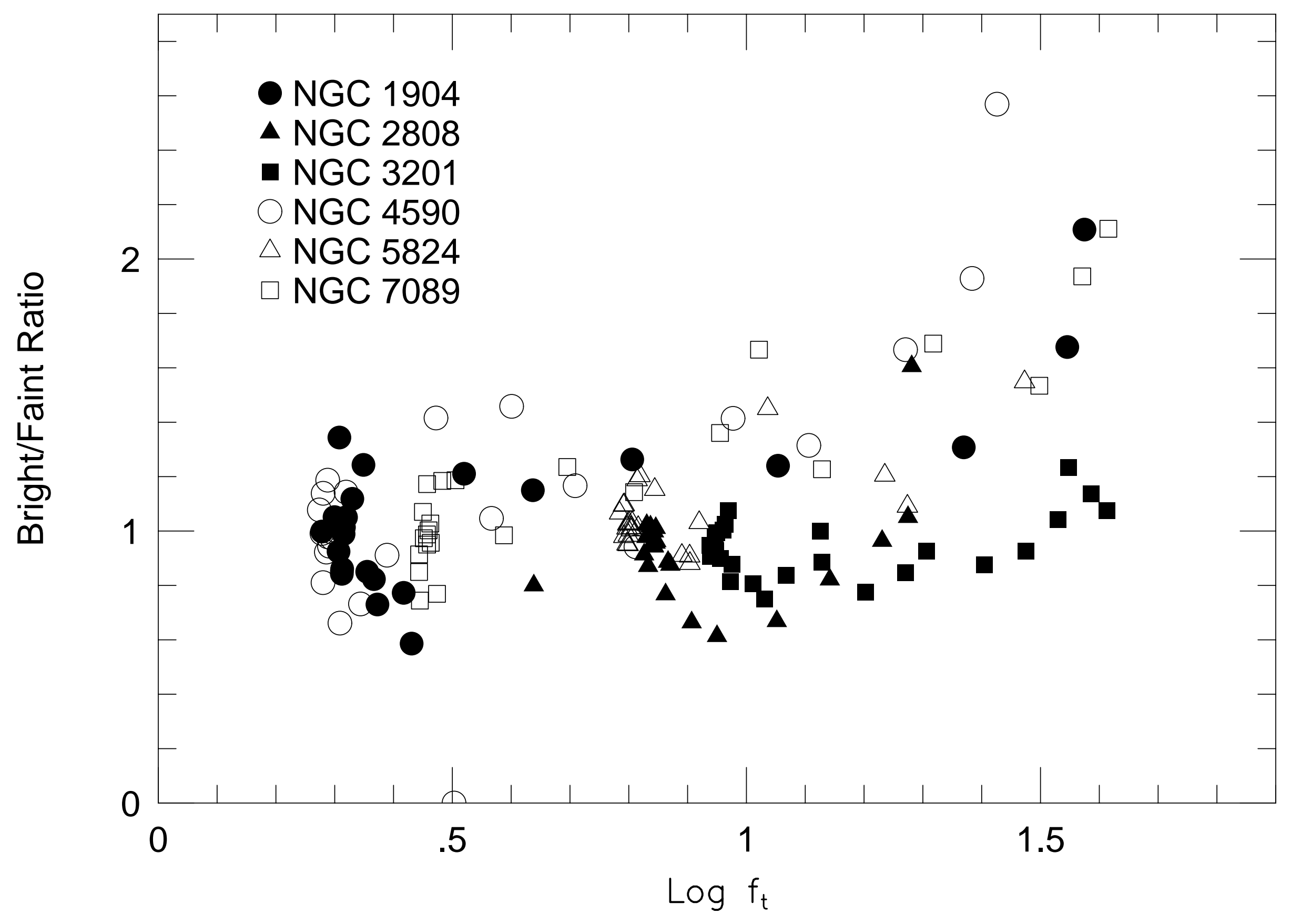




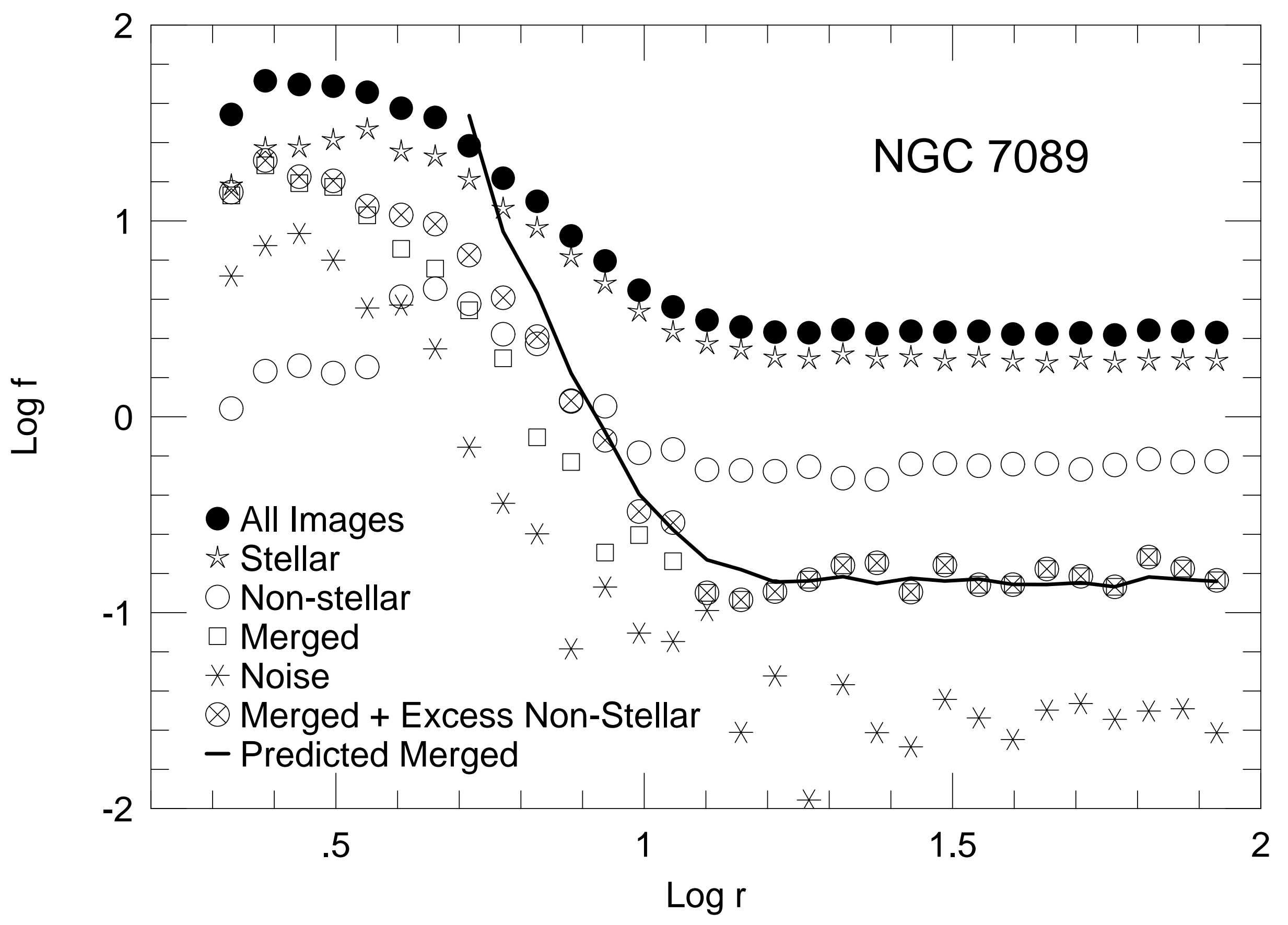




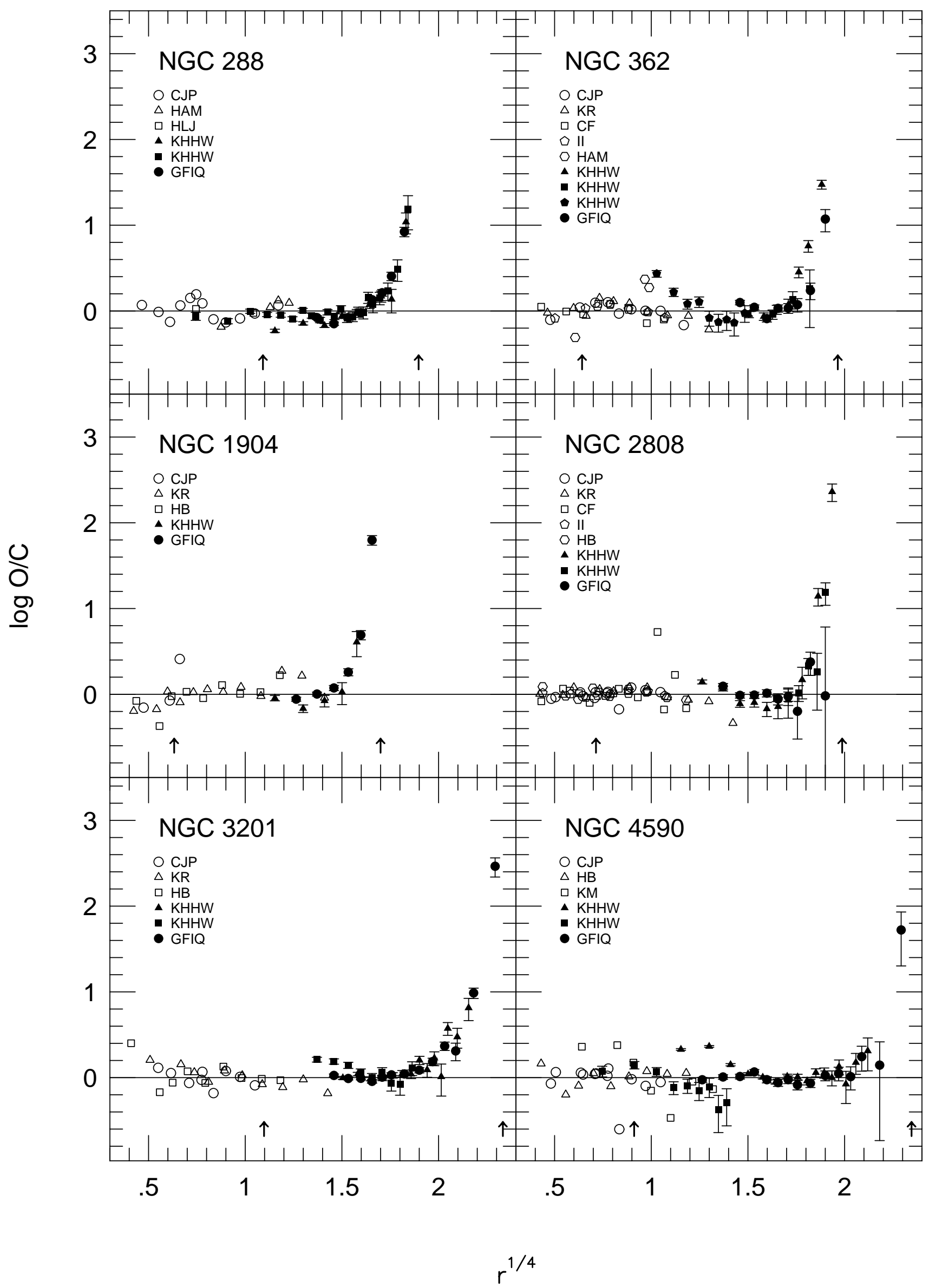




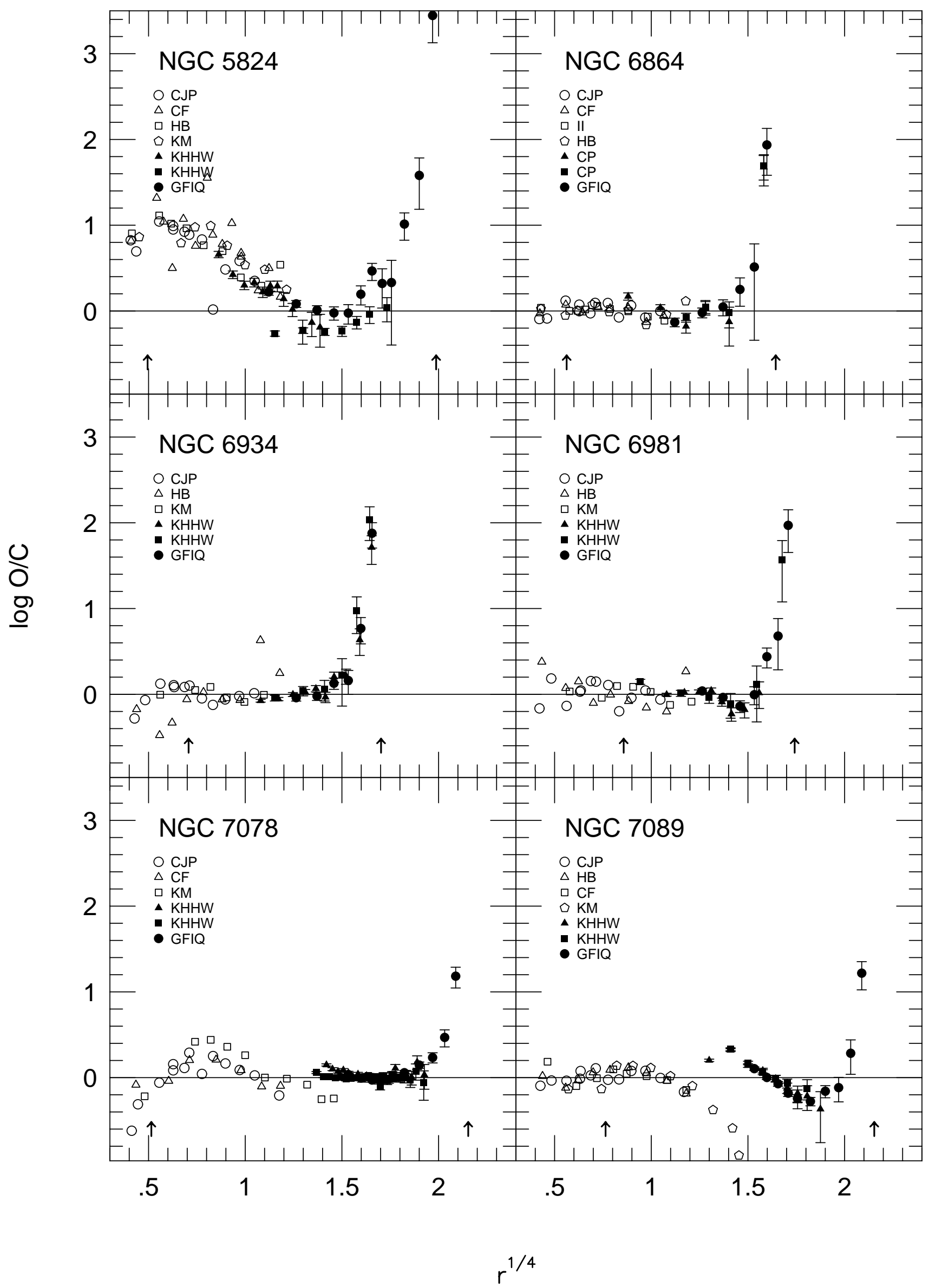




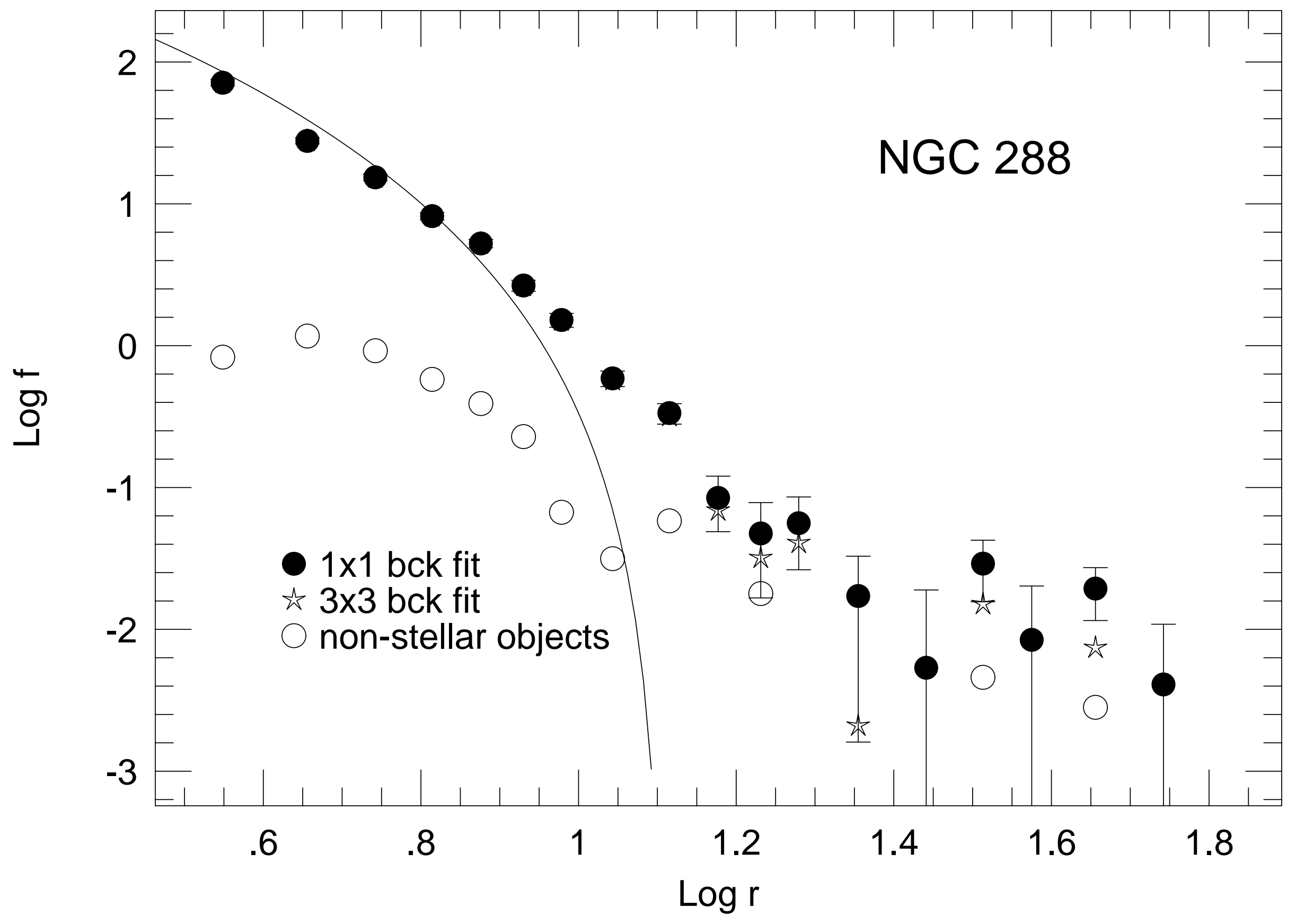




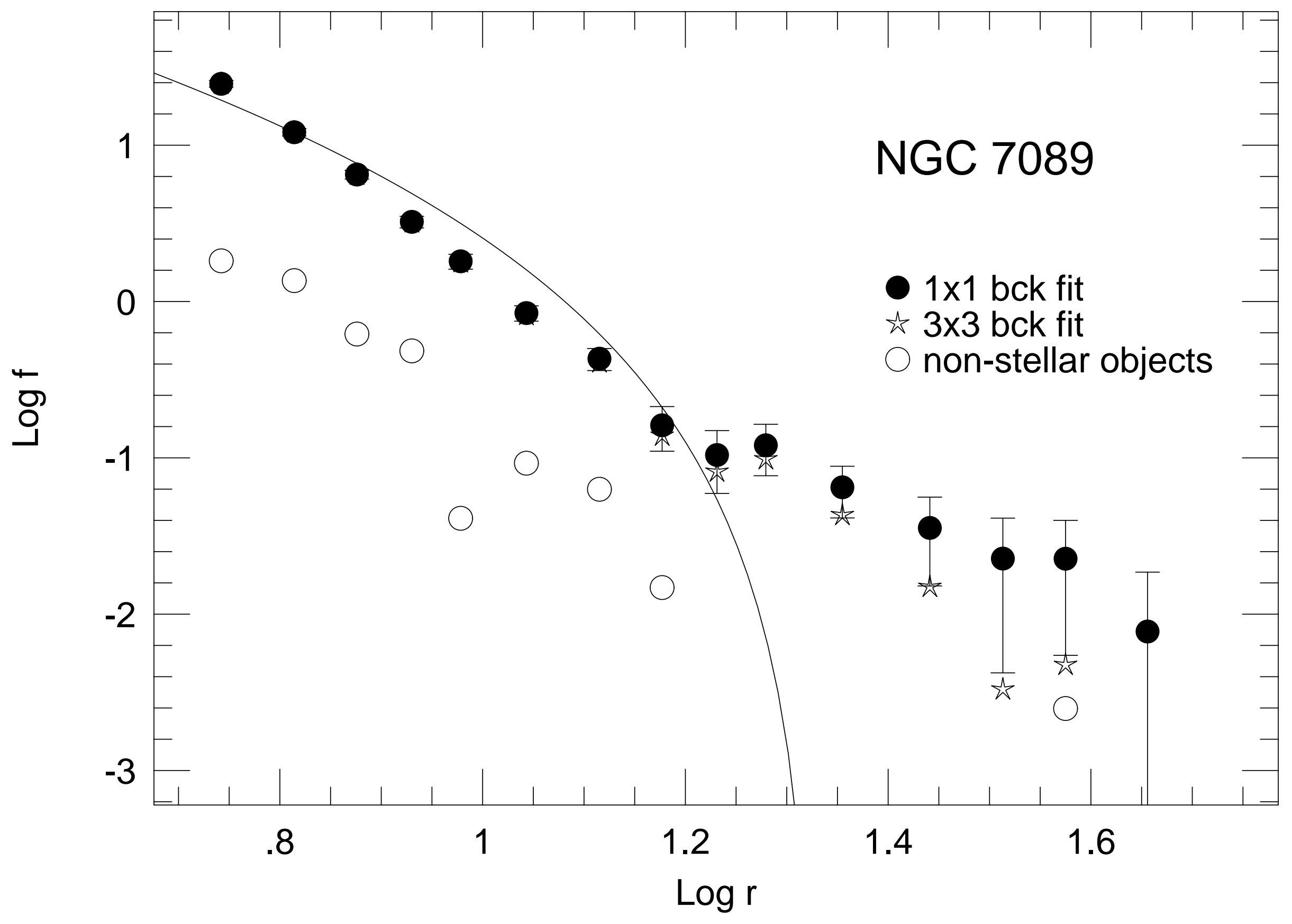



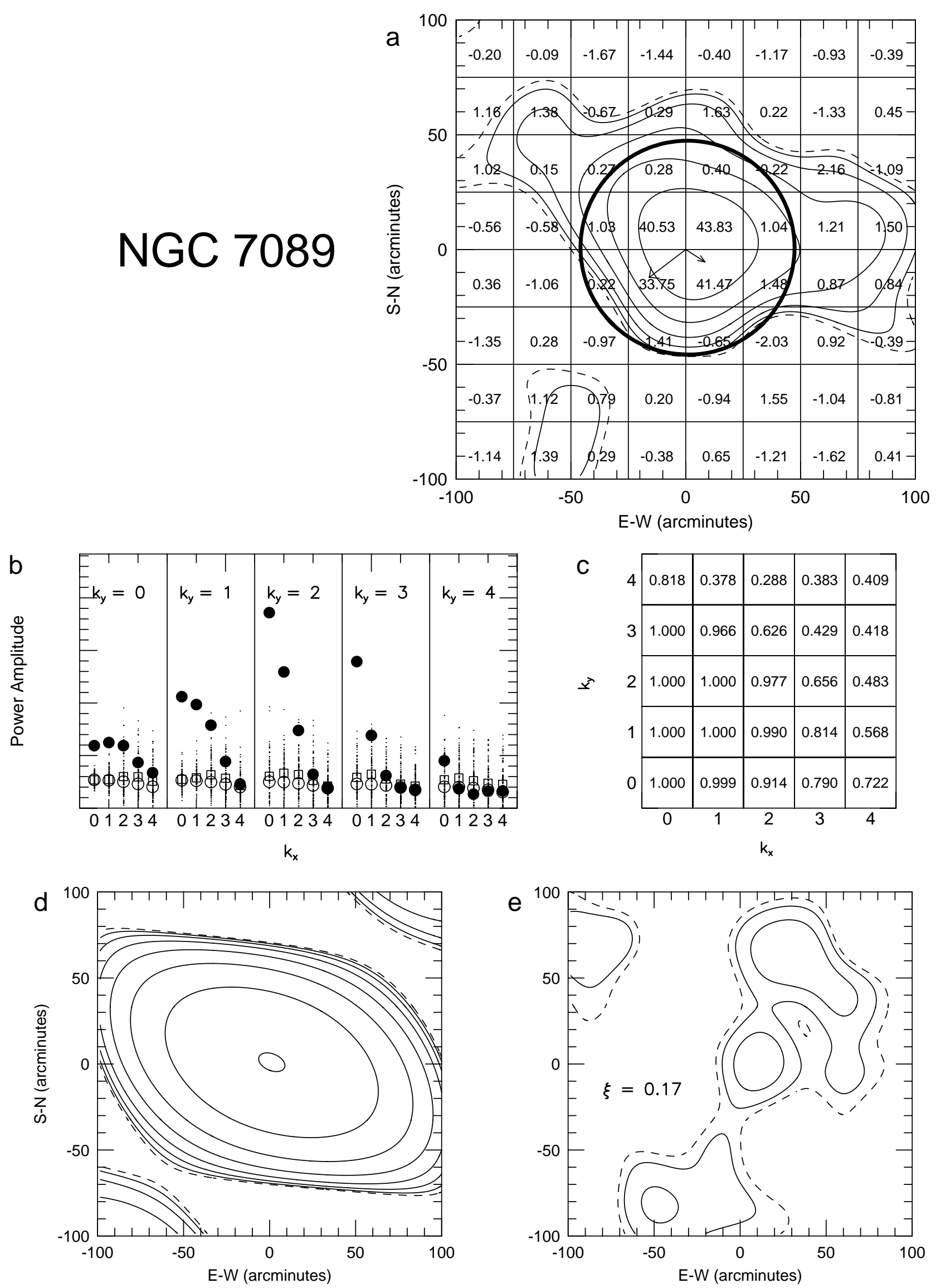


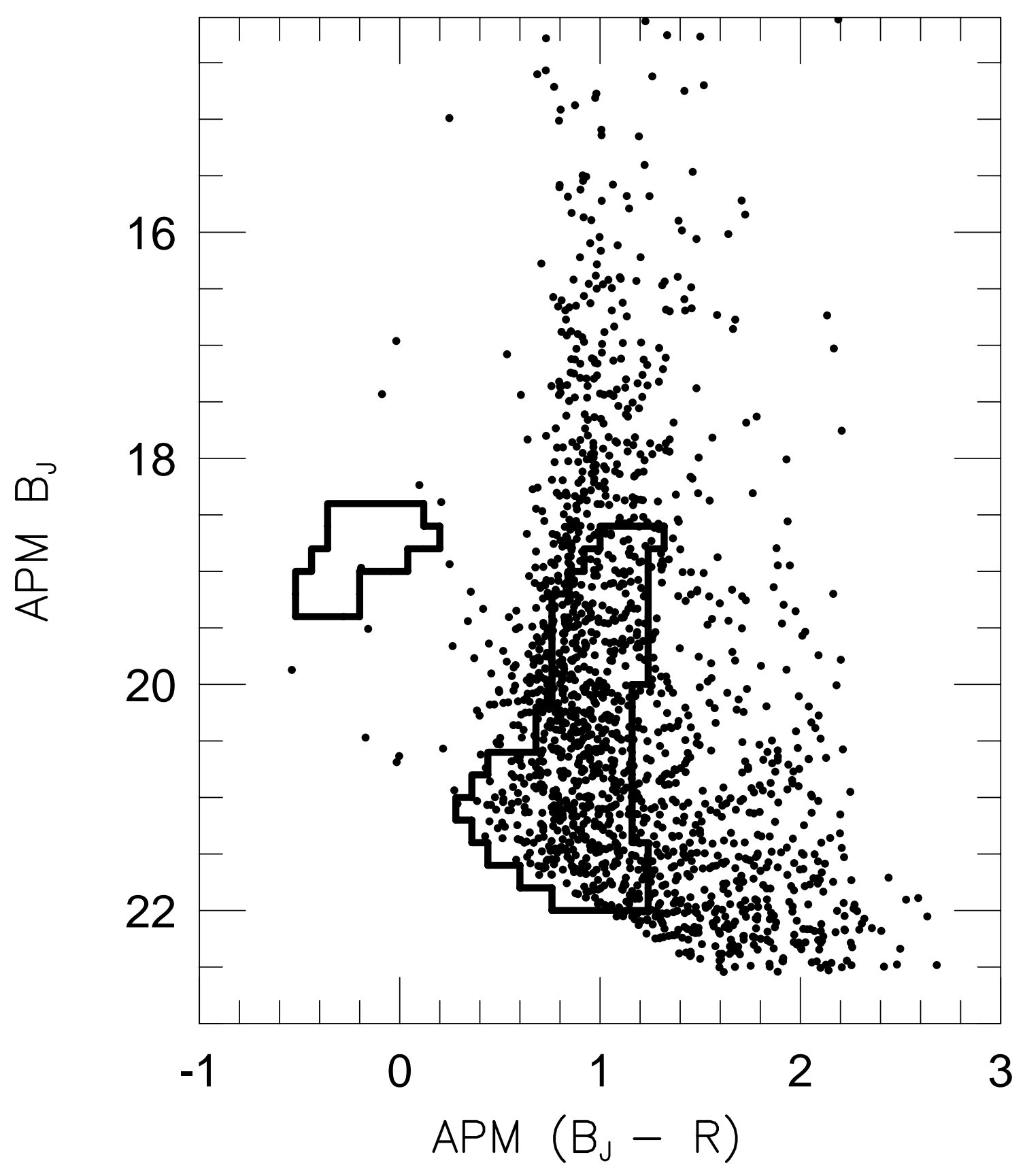




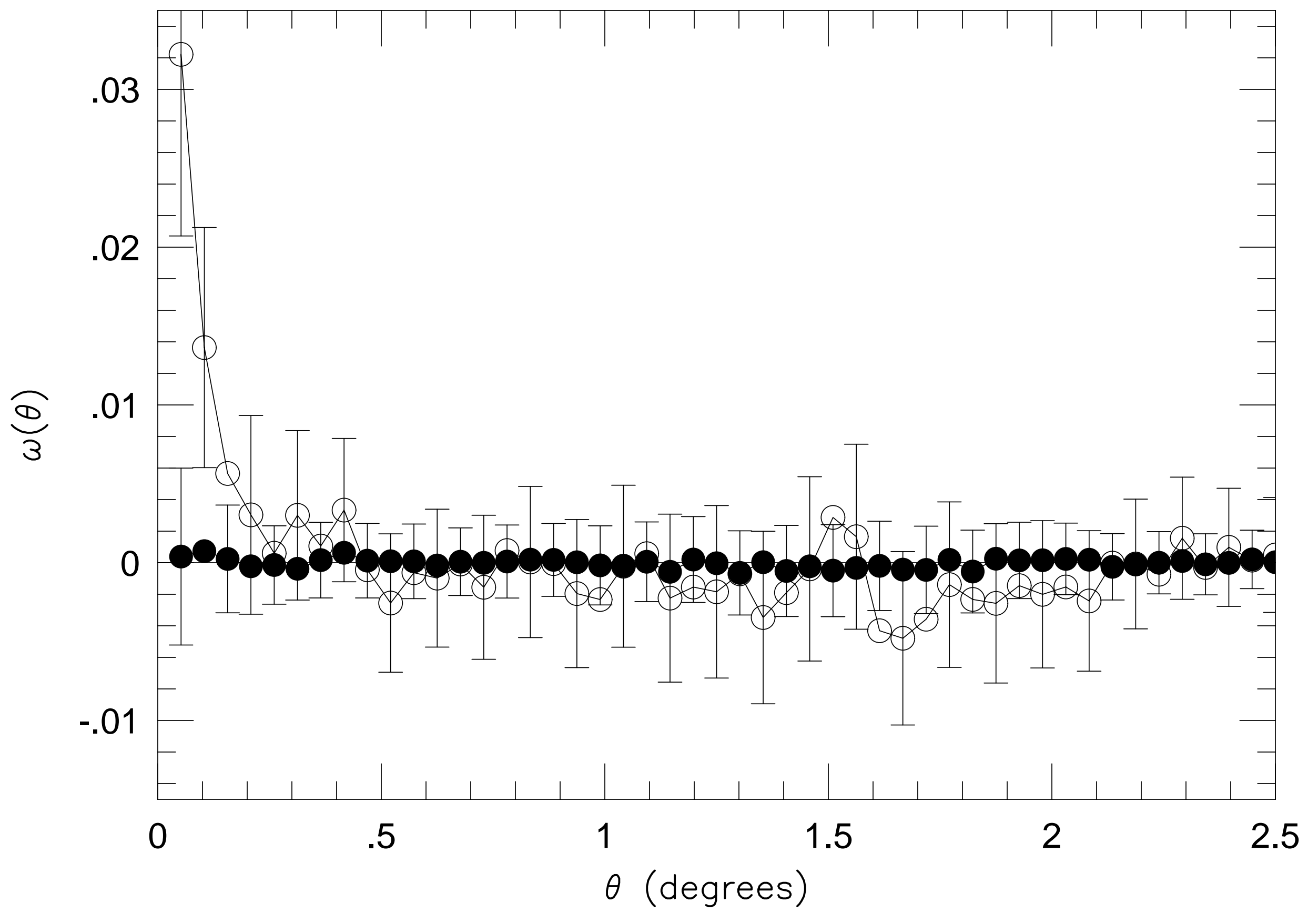




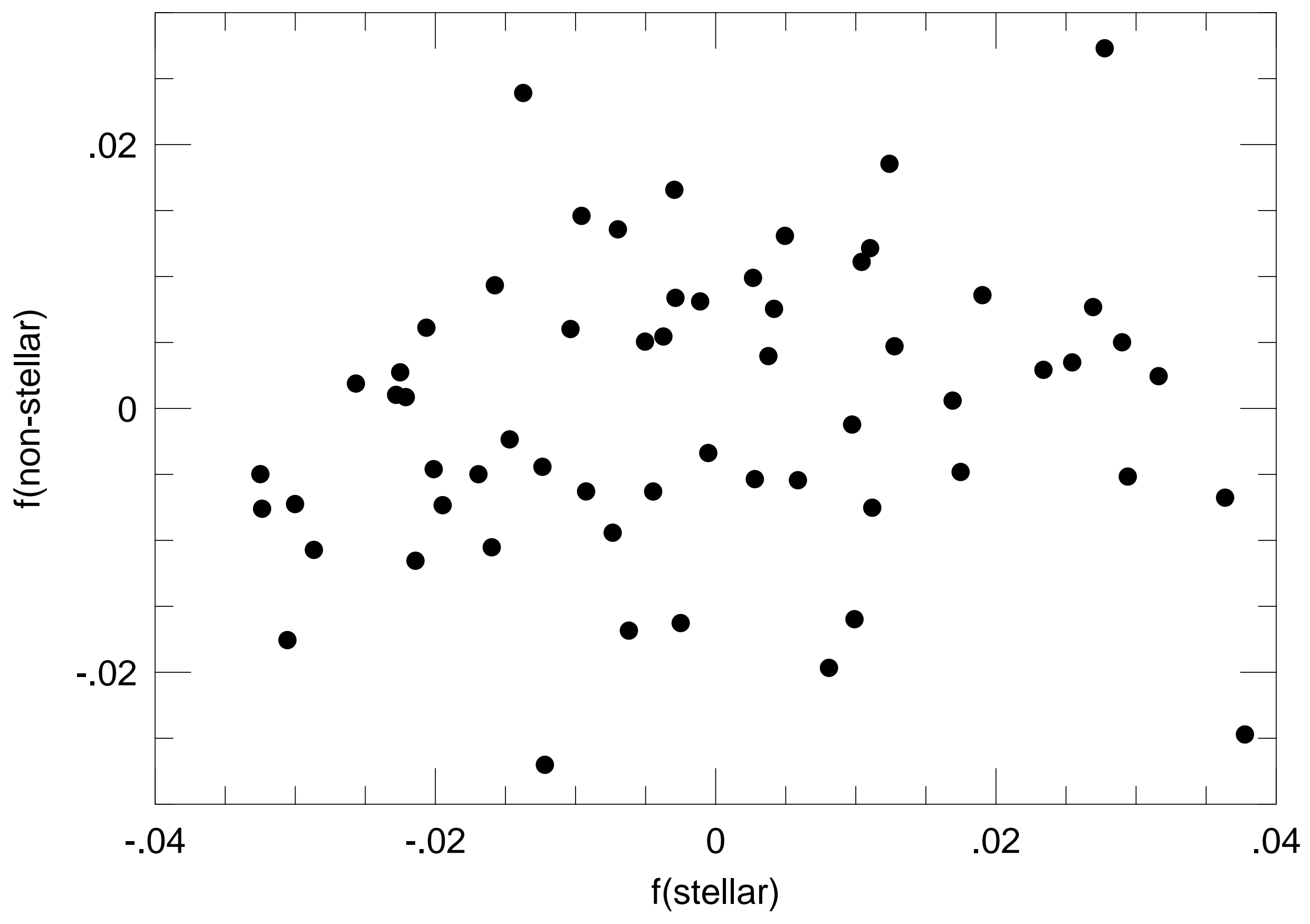




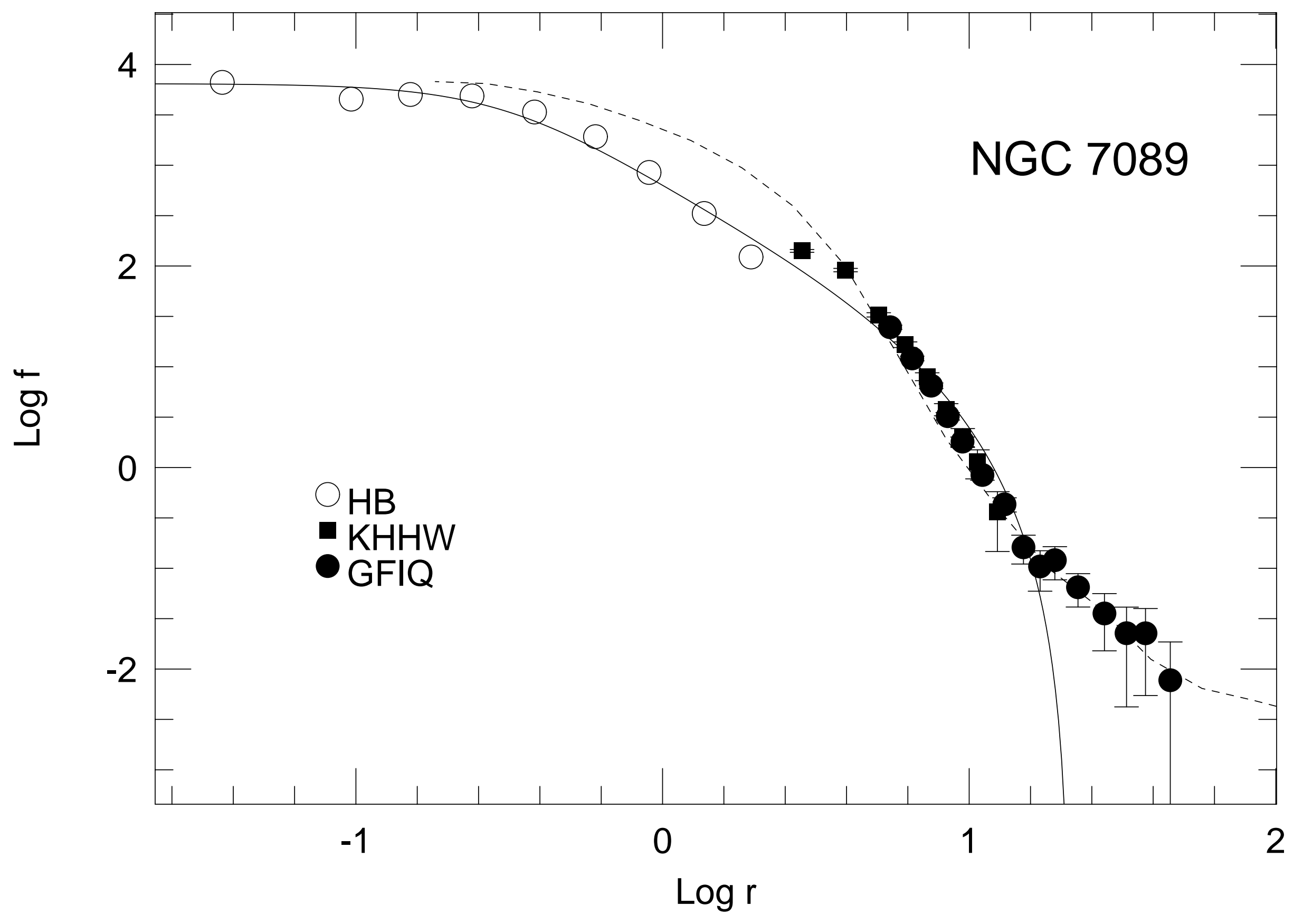




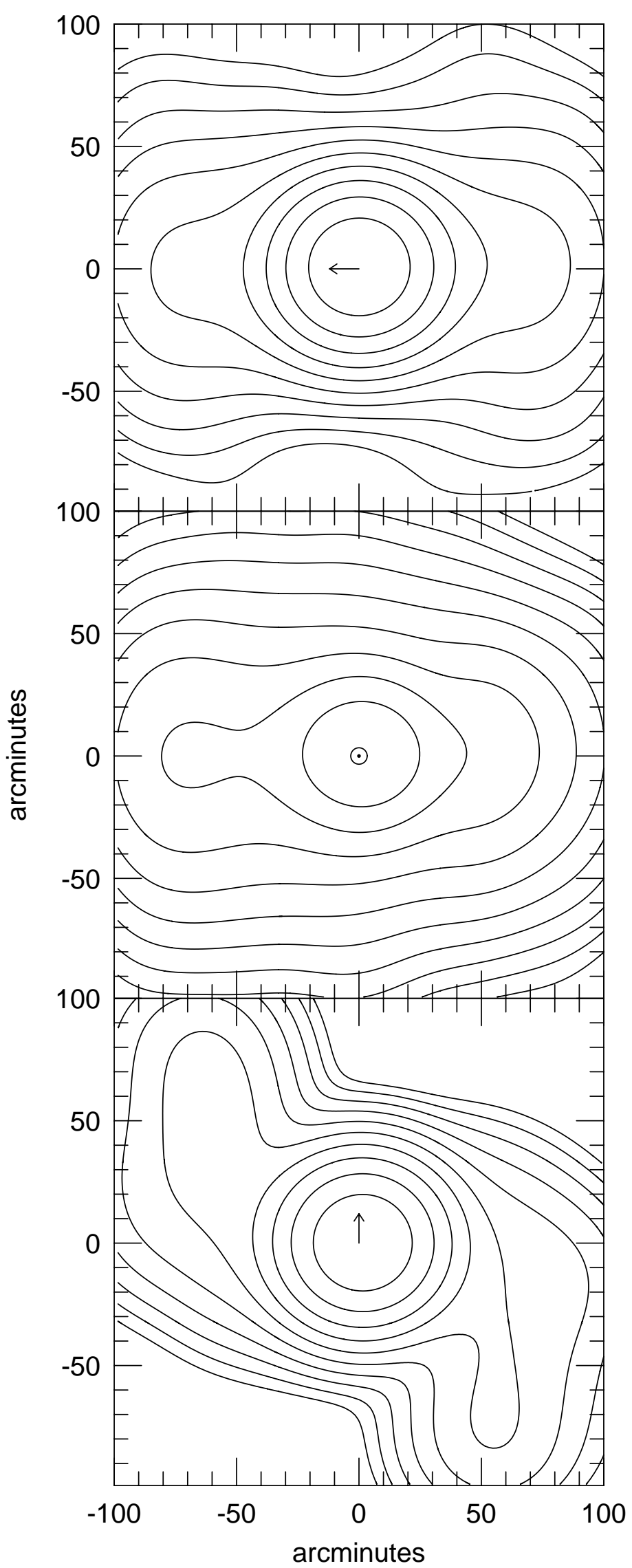




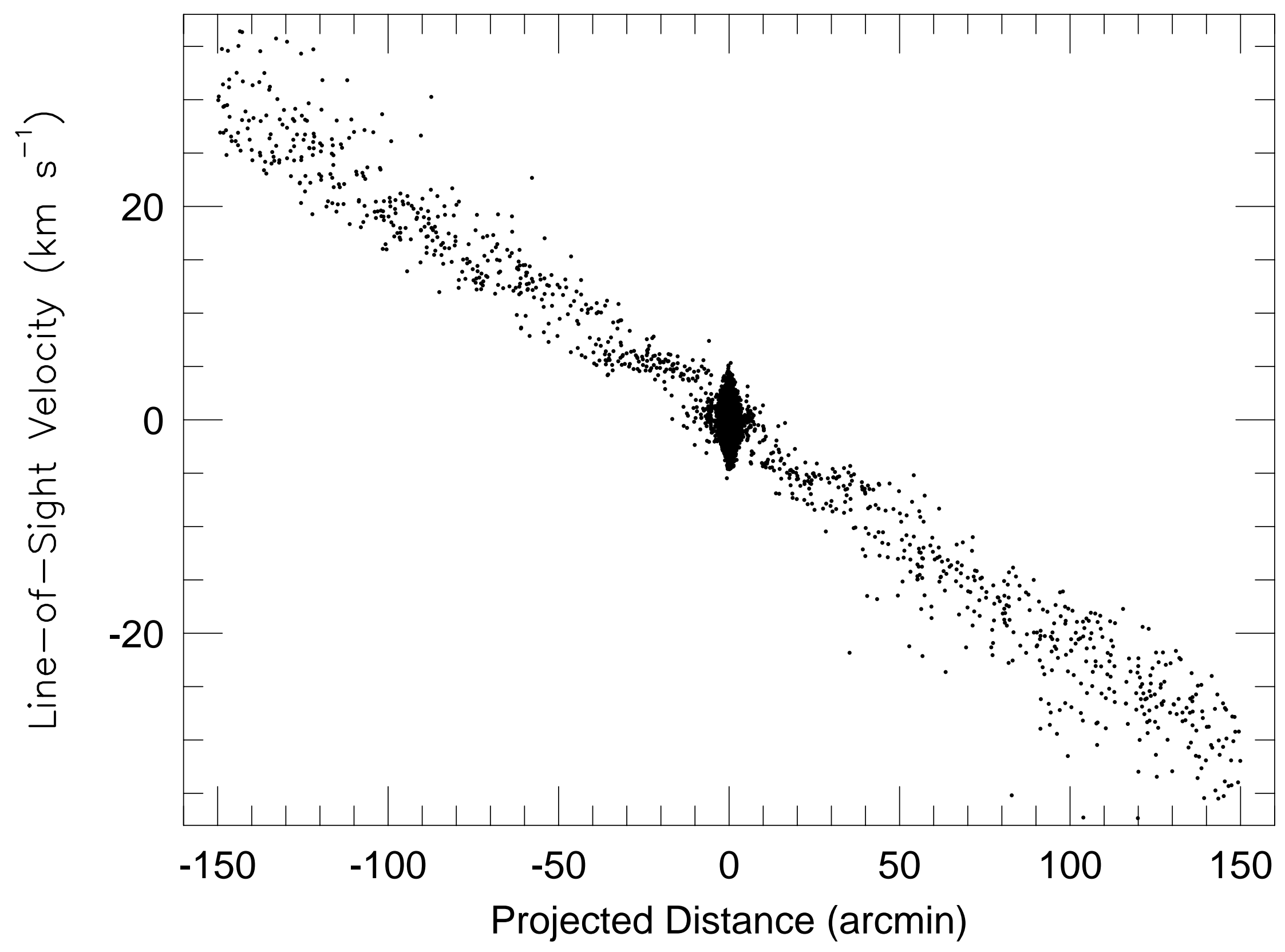




\title{
Globular Clusters with Tidal Tails: Deep Two-Color Star Counts
}

\author{
Carl J. Grillmair ${ }^{1}$, K. C. Freeman \\ Mount Stromlo and Siding Springs Observatories, Australian National University, Private \\ Bag, Weston Creek Post Office, ACT 2611, Australia \\ M. Irwin \\ Institute for Astronomy, Madingley Road, Cambridge, CB3 0EZ, United Kingdom \\ P. J. Quinn \\ Mount Stromlo and Siding Springs Observatories, Australian National University, Private \\ Bag, Weston Creek Post Office, ACT 2611, Australia
}

\begin{abstract}
We examine the outer structure of 12 Galactic globular clusters using star-count analyses. Deep, two-color, photographic photometry is used to select and count stars with colors and magnitudes consistent with clusterspecific, color-magnitude sequences. The resulting reduction in the number of contaminating foreground stars allows us to push the star counts to significantly lower surface densities than has previously been possible. We find that most of our sample clusters show extra-tidal wings in their surface density profiles. The form of the surface density profiles is consistent with recent numerical studies of the tidal stripping of globular clusters. Two-dimensional surface density maps for several clusters are consistent with the expected appearance of tidal tails, with allowance for the effects of orbit shape, orbital phase, and orientation of our line of sight. We identify the extra-tidal material with stars which are still in the process of being removed from the clusters. The extra-tidal stars effectively limit the accuracy to which we can resolve the "tidal" radii of globular clusters. However, by tracing the orbital paths of their parent clusters, these stars may also facilitate a renewed attack on the problem of determining globular cluster orbits and the shape of the Galactic potential field.
\end{abstract}

Subject headings: globular clusters: general - stellar dynamics - Galaxy: structure - Galaxy: halo - Galaxy: kinematics and dynamics - astrometry

\footnotetext{
${ }^{1}$ Current Address: Lick Observatory,University of California, Santa Cruz, CA 95064
} 


\section{Introduction.}

Globular clusters first demonstrated their usefulness as tracers of Galactic structure when Harlow Shapley located the center of the Galaxy by studying their distribution in space (Shapley 1918). Since that time, globular clusters have in many ways become the cornerstone for our understanding of the formation, structure, and dynamics of the halo of our Galaxy. We were initially motivated to undertake a study of the limiting radii of globular clusters by the curious finding of Seitzer (1983) (see also Freeman and Norris 1981) that the metallicities of clusters appeared to be correlated with their orbital radii at

perigalacticon, $R_{m i n}$. Clusters whose orbits are believed to take them nearer to the Galactic center generally have higher metallicities. Such a correlation is not nearly as evident in a plot of metallicity against present Galactocentric distance, and one possible inference is that clusters may have actually formed near their perigalactica (Freeman and Norris 1981). Since the tidal radii of globular clusters depend in some manner on the perigalactic distances of their orbits, our hope was to improve on existing determinations of $R_{\text {min }}$ and hence to both verify and examine more closely this relationship with metallicity.

It has long been supposed that globular clusters must have a finite edge due to the removal of stars by the Galactic tidal field. However, establishing the radius at which cluster surface densities actually vanish has proven difficult. Owing to the remarkable agreement over several orders of magnitude between the projected surface densities predicted by King models (King 1966) and most observed cluster surface density profiles, limiting radii of globular clusters have traditionally been estimated using the model-predicted value of the King tidal radius, $r_{t}$. Based on the postulates that globular clusters are limited in extent by the Galactic tidal field and that the limiting radii are established at the perigalactic points of cluster orbits (von Hoerner 1957; King 1962), Peterson (1974) attempted to put constraints on the shapes of globular cluster orbits using the then published values of $r_{t}$. The results of this work, while suggestive, were not entirely credible since the inferred perigalactic distances for several clusters were found to be greater than their present Galactocentric distances. Innanen, Harris, \& Webbink (1983) carried out a similar study and concluded that globular cluster orbits were rather more circular than an isotropic velocity distribution would require. However, their attempts to determine individual cluster perigalactica were defeated, and they cited the large uncertainties in published values of $r_{t}$ as being chiefly responsible.

In this paper we use the method of star-counts to examine the peripheral structure of a sample of Galactic globular clusters. Star-count analyses are statistically superior to aperture photometry at large radii where the ratio of cluster surface brightness to sky brightness becomes smaller than the ratio of cluster stars to foreground stars. Moreover, 
ignoring noise contributions from the foreground stars, the star-count signal-to-noise ratio goes as $\sqrt{N}$, where $N$ is finite, while the noise associated with aperture photometry can be heavily influenced by relatively few luminous giant stars.

Assuming for the moment that $r_{t}$ should actually correspond to the real, physical limits of clusters, its fitted value is heavily influenced by the superior statistics available at relatively small radii. The literature contains several examples in which the star counts near $r_{t}$ apparently depart from the form predicted by the King model which best fits the bulk of the data (Peterson 1976; Illingworth \& Illingworth 1976). Indeed, Peterson \& King (1975) note several instances in which King models do not fit the star-count profiles at all. In view of the relatively minor consequences for studies of internal cluster dynamics, these departures have never been seriously followed up.

The largest source of uncertainty in attempting to measure surface density profiles near the limiting radii of globular clusters results from simple Poisson statistics introduced by the overwhelming number of contaminating field stars. An obvious way to reduce the number of field stars in a given sample is to count only stars which have colors and magnitudes which resemble those of cluster stars. Prior to embarking on this project, we carried out extensive simulations to determine the extent to which tidal radius determinations could be improved by being so selective. Using King model surface density distributions, canonical cluster color-magnitude sequences, and field star distributions computed from the Bahcall-Soneira model (Bahcall \& Soneira 1980; Mamon \& Soneira 1982), the simulations revealed that a large fraction of the field stars could indeed be eliminated from the counting process. Combining color-selection with a more direct method of fitting the surface density profile at large radii, we found that the uncertainty in the fitted value of $r_{t}$ could be reduced by as much as an order of magnitude. The working assumption in these simulations was that the cluster surface density profiles at large radii could be well represented by King models...

The observational material used for this study is described in Section 2. Star counts, residual foreground removal, and crowding corrections are discussed in Section 3. In Section 4. we examine the observed structure of globular clusters at large radii. We briefly compare our observational findings with recent results of numerical simulations in Section 5.

\section{Observations.}

The sample we have chosen to study consists of 12 Galactic globular clusters with current Galactocentric distances in the range $9<R_{G C}<40 \mathrm{kpc}$. These clusters are 
particularly interesting in that their metallicities appear to be related to their perigalactic distances, as inferred from their fitted tidal radii (Freeman and Norris 1981). Table 1 lists relevant data from the literature concerning these clusters. Coordinates are taken from Shawl \& White (1986). Core and tidal radii are from the compilation of Trager, Djorgovski, \& King (1993), and values for $R_{G C}$ are taken from the compilation of Djorgovski (1993).

Our simulations revealed that, in most cases, the accuracy to which we could resolve the tidal cutoffs of model clusters depended upon pushing the star counts as far down the main sequence as possible. This is purely a consequence of the large rise in the luminosity function at faint magnitudes and the correspondingly improved counting statistics. Exceptions to this general rule were dictated by the predicted onset of excessive numbers of field stars within some envelope containing the cluster-specific color-magnitude sequence. These exceptions included clusters situated (in projection) near the Galactic plane (NGC 2808, NGC 3201) or the Small Magellanic Cloud (NGC 362).

Despite the advent of large-format CCDs, the apparent sizes of globular clusters are so large that the only practical way of carrying out a study of this type is through the use of the photographic Schmidt plate. Our desire to go as deep as possible, combined with the fact that none of the sample clusters is well-centered on existing Survey plates, prompted us to apply for suitable plate material to be taken with the United Kingdom Schmidt Telescope (UKST) at Siding Springs Observatory. Thanks to the dedicated efforts of the staff of the UKST, some 50 high-quality plates were acquired in the space of just over a year. The details concerning these plates are given in Table 2. Whereas most cluster plates were sky-limited, the exceptional fields noted above required exposure times limited to about 30 minutes. By request, the plates were typically taken in conditions during which the seeing FWHM was $>2^{\prime \prime}$. In addition to improving the photometric accuracy, this enabled us to take advantage of periods during which seeing conditions were inadequate for higher-priority Southern Survey plates. On the other hand, it also meant that crowding of stellar images near the cores of the clusters would be correspondingly more severe. Two plates were acquired in each of $B_{J}$ and $R$ to improve the photometry at faint magnitudes.

The plates were scanned using the the Automatic Plate Measuring System (APM, (Kibblewhite et al. 1984). This system has been in operation for over a decade and has become an invaluable resource for large-scale photographic survey work. Its primary features include a laser spot scanner capable of scanning an entire Schmidt plate in about 2 hours, as well as extensive on-line processing capabilities. The actual scanning and processing procedures have been discussed at length by Irwin \& Trimble (1984) and Bunclark \& Irwin (1983) and are detailed here only as they become relevant.

Processing of the plate scans was carried out using a crowded-field algorithm (Irwin 
1985) so as to push the star counts as far into the cores of the clusters as possible. Based on areal profiles and second order moments, detected images were classified as either stars, merged images, non-stellar sources, or noise. For each plate, scanning was carried out for an area about $4^{\circ}$ square and centered on the cluster. The total number of detected images ranged from 60,000 to 400,000 per field.

\section{Star Counts.}

\subsection{Identification of Cluster Stars.}

In Figure 1 we show color-magnitude diagrams, based on the APM magnitudes of images classified as stars, both for the central regions of each cluster and for annuli extending outwards from well beyond the published tidal radii. The APM magnitudes are linearly related to real apparent magnitudes over essentially the entire range of magnitudes shown in these figures (Bunclark \& Irwin 1983). Where we have two or more magnitudes available per image in each color, we have used the average value. Images for which we have no color information (due, for example, to differences in image size or background density between $B_{J}$ and $R$ plates) are not used. The cluster sequences, particularly near the turnoff region, are quite distinct. Based on the width of the main sequences, we estimate our magnitude uncertainties to be about $0.2 \mathrm{mag}$ at the limit of the deepest plates. The zero-point calibration is approximate but is essentially irrelevant for the purposes of establishing cluster membership.

Shown as heavy lines in Figure 1 are the regions within which stars are considered to be likely cluster members for counting purposes. Whereas the color-magnitude sequences evident in the left-hand panels of Figure 11 rely on a few of the most crowded and least well-measured stars on each plate, defining the color-magnitude envelope is carried out for a much larger number of stars over a much larger area of sky. The color-magnitude envelopes are empirically chosen so as to optimize the ratio of cluster stars to field stars in the relatively sparsely populated outer regions of each cluster. This is done by subdividing the color-magnitude plane into a $50 \times 50$ array in which individual elements are 0.08 magnitudes wide in color and 0.2 magnitudes high in APM $B_{J}$. Assuming that the color-magnitude distribution of the field stars does not vary across the plate, an APM color-magnitude sequence for each cluster can be estimated from

$$
f_{c l}(i, j)=n_{c l}(i, j)-g n_{f}(i, j)
$$


where $n_{c l}, n_{f}$ refer to the number of images with color index $i$ and magnitude index $j$ counted within the central region of the cluster and in an annulus well outside the cluster, respectively. $g$ is simply the ratio of the area of the cluster annulus to that of the field-star annulus. We compute the "signal-to-noise" ratio for each color-magnitude element

$$
s(i, j)=\frac{f_{c l}(i, j)}{\sqrt{n_{c l}(i, j)+g^{2} n_{f}(i, j)}} .
$$

The $s(i, j)$ array is then smoothed according to the estimated color and magnitude errors in each element. Errors in $B_{J}$ and $R$ as a function of magnitude are estimated by comparing the magnitudes determined from different plates of the same color. Each element of the color-magnitude array is then convolved with a bivariate, Gaussian smoothing kernel with dispersions in the $B_{J}$ and $B_{J}-R$ dimensions set to be equal to the appropriate errors. Figure 2 shows a contour map of the smoothed distribution of $f_{c l}(i, j)$ for NGC 3201. Shown as a heavy line is a contour of constant signal-to-noise ratio, $s(i, j)$ (smoothed), arbitrarily chosen to match the contours of $f_{c l}(i, j)$ on the red side of the cluster sequence. The slight blueward bias of $s(i, j)$ is a consequence of the relatively low density of field stars blueward of the cluster sequence (e.g. Figure 1), and the presence of disproportionate numbers of field stars to the red.

The optimal range of colors and magnitudes for each cluster were determined as follows. (i) The elements of $s(i, j)$ were sorted into descending order over the one-dimensional index l. (ii) Beginning with the array element with the highest signal-to-noise ratio $(l=1)$, star counts were carried out in an test annulus extending over $r_{t} / 2 \leq r \leq r_{t}$ using progressively larger areas of the color-magnitude diagram $a_{k}=k a_{l}$, where $a_{l}=0.016 \mathrm{mag}^{2}$ is the area of a single element in the color-magnitude array. (iii) Field-star surface densities were determined as a function of $a_{k}$ in the same manner. (iv) The cumulative signal-to-noise ratio, $S\left(a_{k}\right)$, was computed from

$$
S\left(a_{k}\right)=\frac{N_{c l}\left(a_{k}\right)-g N_{f}\left(a_{k}\right)}{\sqrt{N_{c l}\left(a_{k}\right)+g^{2} N_{f}\left(a_{k}\right)}}
$$

where

$$
N_{c l}\left(a_{k}\right)=\sum_{l=1}^{k} n_{c l}(l)
$$




$$
N_{f}\left(a_{j}\right)=\sum_{l=1}^{k} n_{f}(l)
$$

and $n_{c l}(l)$ now refers to the number of images within the test annulus having ordered color-magnitude index $l$. In principle one could normalize the distribution of stars near the cluster center to the surface density of cluster stars in the outer regions (rather than counting stars in a test annulus) to achieve a statistically smoother result, but one would then have to contend with crowding and luminosity effects which are themselves functions of surface density. In either case, the lower cluster-star surface density has the effect of moving the peak in the $S\left(a_{k}\right)$ function to smaller color-magnitude envelopes. Figure 3 shows the run of $S$ with increasing color-magnitude area $a_{k}$ for the case of NGC 362. As is apparent in Figure 1, the color-magnitude sequence of NGC 362 is hemmed in by foreground Galactic stars and the giant branch and blue main sequence of the Small Magellanic Cloud. $S$ consequently drops quite quickly as one moves laterally away from the cluster sequence. (v) Based on the peak value of $S$ as read from plots such as Figure of, a threshold value of $s \geq s(i, j)$ is determined, yielding one or more regions in the color-magnitude array in which the contrast between the surface densities of cluster and field stars is highest. (vi) Minor editing of the perimeter of the optimal color-magnitude envelope was carried out to remove outliers (e.g. single-star events in areas of the color-magnitude diagram where the field-star density is low) and to smooth the contours of the selected area.

In several instances, the color-magnitude envelopes ultimately selected included only stars at and below the turnoff and, occasionally, on the horizontal branch. This reflects both the rise in the luminosity function at faint magnitudes and the fact that the cluster giant branches often extend into regions of the color-magnitude diagram heavily populated by disk main-sequence stars. Blue horizontal branches fall well clear of the main body of foreground stars and thus provide an inexpensive additional source of counts. While some of the color-magnitude sequences in Figure 1 may appear somewhat ratty due to high field-star contamination, severe image crowding, and plate saturation, the selected color-magnitude envelopes do not rely on these sequences and are considerably more secure. Our final color-magnitude envelopes reduce foreground contamination by between $41 \%$ (NGC 3201) and 92\% (NGC 7078).

\subsection{Modeling the Distribution of Field Stars.}


Each $\approx 4^{\circ}$ square scan was subdivided into an array of $128 \times 128$ elements. Surface densities were computed by simply counting the number of images in each element and dividing by the appropriate area. All images (rather than just those classified as stars) were counted owing to the rather unpredictable performance of the image classification algorithm in regions of high surface density. In regions of low surface density, the inclusion of images classified as non-stellar contributed to the Poisson uncertainties in the counts. However the mean surface densities of non-stellar images in regions beyond the published tidal radii never constituted more than $25 \%$ of the total so their contribution to the counting statistics is relatively minor. The contribution of galaxies to the variation in surface density across the field is considered in Section 4.2 .

Foreground contamination was modeled by masking the central region of each plate scan (generally the region within $1.5 r_{t}$ ) and fitting a low-order, bivariate polynomial to the remaining area. This polynomial has the form

$$
z(x, y)=\sum_{i=0}^{k} \sum_{j=0}^{l} a_{i j} x^{i} y^{j}
$$

where $k$ and $l$ refer to the degree of the polynomial in the $x$ and $y$ directions, respectively. Thus, a $1 \times 1$ polynomial fit would be a twisted plane in which the $z(x, y)$ varies linearly along any given row or column.

The star counts near the limiting radii of globular clusters are sensitive to the form of foreground subtraction used. Historically, star-counts have been foreground-subtracted using the surface density, integrated around an annulus, at a radius deemed large. In the absence of foreground density fluctuations on scales similar to that of the cluster itself, and assuming that the determination of the foreground density is indeed carried out well beyond the limits of the cluster, this method should in principle be perfectly adequate. However, as we shall see in Section 4. variations in the distribution of foreground stars can be quite dramatic, whether due to differential extinction near the galactic plane or significantly non-linear surface density gradients across the field. An extreme example is that of NGC 362, which is projected against the northern extension of the Small Magellanic Cloud.

The order of the surface fits were chosen so that the resulting sky arrays are dominated by the smooth gradient in field density due to underlying structure of the Galaxy. In Figure 4 we compare the model foreground distribution computed for the field surrounding NGC 7089 using $1 \times 1$ and $3 \times 3$ polynomials. The cross terms in Equation 6 consequently admit variations of 2 nd and 6 th order, respectively. While the 1st-order fit reflects the gradient expected from the Galactic distribution, a 3rd-order fit is heavily influenced by 
local concentrations which may or may not be related to the cluster itself (see below). In all but three cases, a $1 \times 1$ degree polynomial fit was sufficient to remove any large-scale gradients. Fields near the Galactic plane are fitted with $2 \times 2$ bivariate polynomials owing to the underlying exponential increase in field-star densities across the plate. For the field surrounding NGC 362 we use a $2 \times 3$ bivariate polynomial fit to model the surface density distribution of the northern wing of the SMC. In a few instances, small regions of the plate scan containing obvious concentrations of images were masked to avoid skewing the surface fit. With one exception (NGC 7078) the maximum gradients in these surface fits correspond well with the direction to the galactic plane.

Star counts were carried out using a software réseau with concentric annuli one arcminute wide and centered on the cluster coordinates. Since the central $2^{\prime}$ of our clusters are saturated or crowded beyond the ability of the APM software to distinguish individual images, the coordinates of the cluster centers have been taken from Shawl \& White (1986). The raw star counts, along with computed surface densities, associated uncertainties, and appropriate crowding corrections (see Section 3.3.) are given in Tables 3 through 14. The table entries are arranged as follows: The first two columns specify the inner and outer radii of individual annuli in arcminutes. Beyond $9^{\prime}$ the annuli have been combined into progressively larger bins to reduce the relative uncertainties for individual entries. In column 3 are given the effective radii appropriate to each entry. The tabulated radii are area-weighted rather than luminosity weighted (e.g. King 1988), but the surface density gradients over the range of radii with which we are concerned are sufficiently small to make such a distinction insignificant. Column 4 contains the actual number of images counted. In column 5 we tabulate the mean field-star densities determined from surface fitting and corrected for crowding. Crowding corrections, which are given as surface density multipliers, are tabulated in column 6. Note that whereas the human eye is rarely confused by image blending in regions of low surface density and that crowding corrections would not generally be required, machine counts require correction at all radii. We discuss crowding corrections at length in Section 3.3. The sky-subtracted surface densities, corrected for crowding, are given along with their corresponding uncertainties (computed purely from Poisson statistics) in columns 7 and 8.

At the end of each table are indicated the sectors used for the star counts. The réseau used for counting is divided into eight sectors with opening angles of $45^{\circ}$, as shown in Figure 5. In a few instances, certain sectors were avoided owing to either obvious concentrations of images unrelated to the cluster in question or uncertainties in the foreground surface fitting of specific regions. Also indicated at the bottom of each table are the degree of the surface-fitting polynomial used and the average image area $A$ used to compute the crowding corrections. 
The tabulated surface densities are shown plotted in Figure 6 along with normalized star-count profiles of King et al. (1968) and Peterson (1976), and surface densities computed from Peterson's (1986a) compilation of photoelectric aperture photometry. We plot our data

only out to the radius beyond which the computed surface densities are first found to be less than one error bar above zero. Where King et al. provide more than one star-count profile, we have plotted the two profiles with the highest fitted background densities and/or largest radial extents. The aperture photometry data are plotted at their King luminosity-weighted radii. Also shown in these figures are King models computed from the published core and tidal radii (see Table 1), arbitrarily normalized to our counts near the limiting radius. Data sets from the literature are independently normalized to these model-predicted surface densities for all clusters except NGC 5824. Due to the mismatch between the observed and predicted profiles, the aperture photometry for NGC 5824 are instead normalized so as to match the star counts in the region of overlap. References to the original sources of data are given in Table 15.

\subsection{Crowding Corrections.}

Owing to the marginal seeing conditions under which our cluster plates were taken, we are rather severely affected by image crowding at high surface densities. This is evident in Figure 6, where the $\times$ 's denote the raw star counts uncorrected for the effects of image blending. The filled circles result from applying the crowding correction formula derived for machine counts by Irwin \& Trimble (1984)

$$
\alpha_{c}=\frac{f^{\prime}}{f} \simeq \frac{1}{4 A f} \ln (1-4 A f) .
$$

Here $f$ denotes the total observed surface density of images, $A$ is the average image area, and $f^{\prime}$ is an estimate of the true, total surface density. The average image area $A$ depends on the threshold isophote above the local sky used for image detection. An image is defined as a region of simply connected pixels exceeding this threshold, and the image size is consequently affected by such factors as seeing and saturation effects. The value of $A$ is typically a few times larger than the FWHM seeing disk and is best determined by judicious experimentation (see below). If we assume that, to first order, crowding effects are independent of luminosity and color, then we may multiply our observed, color-magnitude-biased surface densities by $\alpha_{c}$ to obtain an estimate of the true stellar surface densities. 
How valid is this assumption? Equation 7 is derived purely on the basis of identical, overlapping images and makes no allowance for possible luminosity effects. It is reasonable to suppose that in areas of high surface density a significant fraction of relatively faint stars could be lost in the photon noise generated by nearby bright stars. Alternatively, faint star counts could be enhanced in these areas owing to the underlying density of unresolved objects and its effect on the threshold used for detection. Consequently, it is of interest to examine our star counts as a function of magnitude to determine the extent to which such effects might be present.

In Figures 0 and 8 we show the variation in the ratio of bright stars to faint stars with radius and total surface density for a representative selection of clusters. The clusters shown have all been observed under similar seeing conditions (FWHM $\left.\approx 2.5^{\prime \prime}\right)$. For each cluster we determine the median magnitude for all images classified as stars and falling within the appropriate color-magnitude envelope, then count the number of stellar images above and below this magnitude. It is apparent from Figures 7 and 8 that the scatter is quite large and, more importantly, that there is considerable variation in the run of the relative numbers of bright and faint stars from cluster to cluster. While the relative fractions of bright stars are sometimes seen to rise near the cluster centers, the onset of this rise does not appear to depend strongly on the total surface density. This is no doubt due in part to the relative densities of field and cluster stars, each having different intrinsic luminosity functions. Interestingly, some clusters show almost no luminosity effects at all.

It should, in principle, be possible to account for the observed luminosity functions of field and cluster stars and so attempt to model consistent luminosity corrections. However, we note that the magnitudes of the variations are relatively small. If we were disposed towards correcting the surface densities by forcing the ratio of bright stars to faint stars to be constant throughout the cluster, then the most offending deviations in Figure 8 would contribute a relatively modest $30 \%$ to the counts. This is smaller than the computed crowding corrections at the same surface density. In addition, we see in Figure 7 that the ratios of bright to faint stars are consistent with unity well within the published tidal radii of these clusters. We consequently ignore luminosity effects and consider the application of Equation 0 directly.

As previously noted, image classification becomes problematic in regions of high surface density. In Figure 9 we show the surface density profiles for images classified as stars, merged images, and non-stellar sources for NGC 7089. Also shown are the densities of merged images one would expect from a straightforward application of Equation 7 . The unreliability of the classification algorithm is demonstrated by the rapid growth near the cluster center in the number of images classified as non-stellar. Assuming that the 
underlying surface density of galaxies should be approximately uniform across the plate, then the apparent overdensity of non-stellar objects near the cluster center may reasonably be attributed to overlapping stellar images. If the intensities of two overlapping stellar images are moderately different, the fainter image may lose its identity and the net, elongated profile will be classified as non-stellar. The $\otimes \mathrm{s}$ in Figure 9 result from combining the densities of objects classified as merged with the densities of non-stellar images in excess of the mean level computed well outside the cluster $\left(r>20^{\prime}\right)$. It is evident that, beyond the region where multiple mergers become important, the number of such "deemed" merged images agrees quite well with the number predicted by Equation 0 .

Equation 7 is obviously quite sensitive to the image size parameter $A$. Unfortunately, we have no reliable, internally consistent method for determining its optimal value. Plots such as Figure 9 provide a rough guide, but owing to complex multiple-merger and saturation effects in the inner regions where we are most sensitive to $A$, their utility is limited. Hence we are forced to rely on the star counts of previous investigators. Specifically, we alter $A$ until our corrected surface density profiles match as closely as possible the profiles of King et al. 's (1968) and Peterson's (1976) data in the inner regions. The correction is largest and most sensitive to $A$ at high surface densities where King et.al's counts are least susceptible to background uncertainties, though we are then subject to the uncertainties in their own crowding corrections. We also note that equation 7 becomes undefined for $f A \geq 4$ and is unlikely to be accurate near this regime. Nonetheless, matching star count profiles by eye result in reasonable values for $A$ (Irwin \& Trimble 1984), and the corrected surface densities shown in Figure 6 generally agree quite well with the counts of King et al. over practically the entire range in common.

\section{Analysis.}

\subsection{Surface Density Profiles.}

Examination of Figure 6 reveals that, while King models generally provide an excellent match to the observations over a large range in surface density, this agreement evidently breaks down at some radius which varies from one cluster to the next. Most clusters show an apparent excess of stars at $r \geq r_{t}$. To better illustrate the departures of the data from model predictions, we show in Figure 10 the observed-minus-computed residuals (in the log domain) using King models with core and tidal radii from Table 1. The residuals are plotted against $r^{1 / 4}$ to better reveal the observed structure at large radii. We are more 
concerned here with gradients in the residuals of individual data sets than with their mean values (which rely on imperfect normalizations of each data set). It is apparent that in all cases, both the star counts of King et al. (1968) and those of the present study depart from the King models at radii considerably less than $r_{t}$. Moreover, the residuals often show systematic trends (i.e. non-zero gradients) even before we reach the positive break-aways which we henceforth ascribe to "extra-tidal" stars. NGC 5824 is a particularly extreme example which clearly cannot be represented with a King model at any radius. Note that luminosity effects and incompleteness due to image crowding (Sec. 3.3.) will have reduced the measured surface densities of cluster stars with respect to the surface density of stars in the field; applying completeness corrections would serve only to increase the apparent departures from King-like behavior.

The slopes in the residuals corresponding to the outer aperture photometry data are no doubt at least partially due to uncertainties in the sky subtraction. Note also that since the total light will be dominated by the contributions of stars on the giant branch but the star counts will be heavily weighted towards stars of lower mass, the form of the residuals could, in some cases, be attributable to mass segregation. While we could investigate this possibility using appropriate multi-mass King models, we are at present less concerned with the internal structure of globular clusters than we are with their limiting radii. If globular clusters are limited in spatial extent by the action of Galactic tidal forces, then we expect the removal of stars (and hence the limiting radii) to be independent of mass.

The outer surface density profiles (Figure 6) of NGC 288, 1904, 5824, 7078 and NGC 7089 show the most significant departures from King-like behavior. Deep plates, high cluster-star surface densities, and low field-star surface densities make these among our best observed clusters. These departures resemble power-laws with slopes which vary from -1.6 (NGC 288) to -5 (NGC 7078). The surface density profile of NGC 5824 is particularly striking in that it appears to follow a power law over almost the entire extent of the data, with some flattening at both small and large radii. With the exception of NGC 2808 (whose outskirts may be heavily obscured - e.g. Faulkner et al. 1992), all of our sample clusters show evidence of at least some extra-tidal material.

Could these extra-tidal extensions be spurious products of inaccurate foreground subtraction? To estimate the effects of our assumed form for the foreground distribution, we fit higher-order surfaces to fields surrounding NGC 288 and NGC 7089. The five-point stars in Figures 11 and 12 show the results of fitting a third-order bivariate polynomial to the foreground distributions in these fields (see also Figure 4 ). While the amplitudes of the extensions are somewhat reduced by virtue of having been modeled out, significant differences between the counts and the King models remain. Evidently, complex and 
highly contrived models would be required to remove the extensions completely. To test the possibility that non-uniformities in the plates have led to local enhancements in the number of detected images, we have reanalysed the data for these two clusters using only the brightest $50 \%$ of the stars within each color-magnitude envelope. We find no significant differences between the resulting profiles and those shown in Figure 6 .

We conclude that the extended profiles are not artifacts of our counting procedures and are consequently real. To what then do we attribute these extra-tidal extensions? Recent numerical simulations by Oh and Lin (1992) and Grillmair et al. (1995) have shown that the removal of loosely-bound cluster stars by the tidal field of the Galaxy is a relatively inefficient process, and that clusters can be surrounded by halos of unbound stars for many Galactic orbits. Moreover, once removed from the cluster, unbound stars form tidal tails extending over enormous distances ahead and behind the cluster in its orbit. The volume densities within the tidal tails are subject to the open-orbit analog to Kepler's 3rd Law; near apogalacticon, the differential slowing of stars in the tidal tails cause the stars in the tails to converge on the cluster. The projected surface densities of tail-stars may increase by an order of magnitude over their values at perigalacticon, depending on the shape of the cluster's orbit and the orientation of the cluster along the line of sight.

If we associate the extra-tidal material with extended halos of stars in the process of leaving the cluster, then the assumed, King-like surface density distribution of bound stars will be affected well within the tidal radius by the column density of the extra-tidal stars along the line of sight. The form of the surface density profile of the extra-tidal material depends on the viewing angle, and it would clearly be hazardous to attempt to model the volume density of extra-tidal material based solely on the observed surface density profile. If cluster distribution functions are truly King-like, then an estimate of the tidal cutoff in the binding energy (which is probably the determining factor in establishing which stars eventually become unbound - Keenan (1981); Grillmair et al. 1995) requires that we avoid fitting models to regions of the surface density profile significantly contaminated by extra-tidal material.

We determine the King-model tidal radius by estimating the radius at which the surface density of extra-tidal material does not contribute significantly to the total. This is clearly a rather subjective endeavor, but the results are not very sensitive to our chosen cutoff due to the distribution of weights, which strongly favors data at small $r$. We estimate core radii by eye using the aperture photometry and determine $r_{t}$ and the scaling constant from our star counts using using a downhill simplex, $\chi^{2}$-minimization routine. Our fitted values for $r_{c}$ and $r_{t}$ are listed in Table 16 . The $90 \%$ confidence intervals are estimated by fitting several hundred Poisson realizations of the star count data for each cluster. 
Having avoided our newly-discovered, extra-tidal extensions, it is not surprising that our fitted tidal radii are generally very similar to those of previous investigators. In several instances our determination of $r_{t}$ is somewhat smaller than the corresponding value in Table 1, a circumstance which we attribute to the inclusion of unrecognized, extra-tidal material in previous analyses. In view of the power-law form evident in the extra-tidal surface density profiles of some clusters (particularly NGC 288, NGC 1904, and NGC 7089), it is difficult to resist the temptation to fit the extensions and the main body of data simultaneously. We have indeed done so, and in some instances found the fitted, King tidal radii to be considerably smaller than the published values, depending on the relative weight of the data at large radii. However, the number of parameters required to model the surface density distribution cannot be justified given our limited knowledge of the distribution of extra-tidal material in the vicinity of the clusters and the orientation of the clusters with respect to our line of sight.

\subsection{Two-Dimensional Structure.}

In panels $a$ of Figure 13 we show contour maps of the smoothed, foreground-subtracted surface densities of images classified as stars in each of our clusters. The surface densities, initially computed over bins of $1.56 \times 1.56$ arcmin, are Fourier-smoothed with a Gaussian kernel with $\sigma=16$ arcmin. The surface densities at $r<r_{t}$ are artificially depressed using a cosine function prior to Fourier transforming to avoid overwhelming the low-density structures beyond $r_{t}$. It is for this reason that the innermost contours often are not centered precisely on the cluster. The heavy, circular contours arise from smoothing (in a manner identical to that used for the real data) projected King models having published core and tidal radii. The model surface densities have been scaled as in Figure 6, and the contours shown are numerically identical to the lowest contours of the real data (shown as dashed lines). Also indicated in Figure 13 are departures from zero of the foreground-subtracted surface densities (in units of the standard deviation) in bins 25 arcminutes square. The standard deviations have been computed from the actual scatter of the mean surface densities of the 48 bins furthest from the cluster center. The arrows at the positions of the cluster centers indicate the direction to the Galactic center. Where more than one arrow is shown, the longer of the two indicates the direction of the measured, absolute proper motion of the cluster (Cudworth \& Hanson 1993).

The smoothed, contour maps show a combination of Poisson fluctuations in the surface density of field stars, unrelated star clusters, and what appear to be extensions of the 
clusters themselves. How significant are these extensions? Could randomly distributed overdensities of field stars masquerade as cluster extensions at this level of smoothing? We have carried out simulations to determine the relative power contained in the low order variations of the surface densities and how this power compares with that expected from random distributions of field stars. Each simulation begins with a $128 \times 128$ array containing the surface densities of all stars meeting our color-magnitude criteria. An appropriate bivariate polynomial (i.e. of the same order as that used in Section 3.) is fitted to the surface densities beyond the tidal radius and subtracted. The surface densities within the tidal radius are left unaltered throughout the simulations since we wish to examine only the power on large scales. The fitted distribution of foreground stars is binned into a $4 \times 4$ array, from which are computed the baseline number of stars in each of the 16 elements. In the course of each simulation, Poisson deviates are generated from these baseline numbers and the appropriate number of stars are randomly distributed across each element. The surface densities of these stars are then modeled and subtracted exactly as above. In this way we preserve any large-scale density gradients in the real data and so simulate the additional effects of fitting and subtracting the foreground distribution. Consider the case wherein strongly non-linear gradients are present in the distribution of foreground stars (near the Galactic plane or the SMC, for example). Subtracting a low-order function from the data would leave higher-order residuals which could conceivably mask underlying extra-tidal cluster structure or, worse, masquerade as cluster structure themselves.

Each simulated, residual surface density map is then Fast-Fourier-Transformed and the power spectral density recorded. A total of 1000 simulations were carried out for each field. In panels $b$ and $c$ of Figure 13 we compare the low-order power of the real data with that of the simulations. The wavenumbers $k_{x}$ and $k_{y}$ correspond to the East-West and North-South directions, respectively, and $k_{x}, k_{y}=0,0$ represents the DC level. In panels $b$, the distributions of simulated power amplitudes (points) are compared with those computed from the real data (filled circles) and with those computed for isolated (i.e. no surrounding field stars) clusters (open circles). The mean values of the simulated distributions are shown as open squares. In panels $c$ are shown the fraction of simulated points with power amplitudes less than those computed from the data. A value of 1.000 indicates that the power amplitude computed from the data exceeds the highest value attained in all 1000 realizations.

It is apparent from these figures that the clusters with noticeable extensions in panels $a$ often have low-order power spectra significantly in excess of that resulting from a purely random distribution of field stars. We can determine whether this power corresponds to the large-scale features evident in panels $a$ by reconstructing the surface density maps using only frequencies containing excess power. In panels $d$ of Figure 13 are shown surface density 
maps generated from the lowest 3 wavenumbers with power amplitudes occurring in less than $0.1 \%$ of the simulations. (Obviously, if no excess power is evident, then an inverse Fourier transform will result in an array of zeros - no contour maps are shown in such cases). The contour thresholds and increments are identical to those used in panels $a$. For the case of NGC 288, we have removed the cluster of images in the northwestern corner of the field before carrying out these simulations. These reconstructions must be interpreted with caution, but the similarities between panels $a$ and $d$ indicate that, in several cases, the general form and scale the extensions are significant at a high confidence level.

While these tests are suggestive, they are by no means conclusive. We have merely tested the hypothesis that Poisson statistics alone could account for apparent large-scale structure. Other factors which could contribute to excess power on large scales include: $(i)$ other clusters of stars in the field, $(i i)$ uncertain image classifications at faint magnitudes which could lead to the inclusion of potentially significant numbers of galaxies in the sample, and (iii) intervening clouds of obscuring material.

In the case of NGC 6864, the relatively high counts extending westwards from the cluster are due to an unrelated concentration of $\sim 200$ disk stars. The concentration is sufficient that we have been able to construct a rough color-magnitude density map (Figure 14) as described in Section 3. Comparing this map with the color-magnitude morphology in the central regions of NGC 6864 (Figure 1) it is apparent that there are comparatively few stars in the region corresponding to the turnoff of NGC 6864, and that horizontal branch stars are not represented at all. Hence we conclude that the extension is not physically associated with NGC 6864. It is unclear how far this "cluster" extends and, while we have specifically avoided the western side of the cluster in our star counts, we view the surface density profile in Figure 6 with some suspicion.

For NGC 288, the high concentration of images in the northwestern corner of the field is due to NGC 253, a giant, early-type spiral in the Sculptor group. In most other cases, while localized concentrations of stars may be apparent, the numbers of stars involved are insufficient to allow us to say anything meaningful concerning their color-magnitude morphology. The contours extending southwards from NGC 362 are suspect owing to the high-order surface fit required to model the distribution of SMC stars.

Given the uncertainties in star/galaxy separation discussed in Section 3.2., it is conceivable that the contours in panels $a$ are influenced to some degree by background clusters of galaxies. On the angular scales over which we are smoothing the distributions of galaxies are intrinsically far more clumpy than the distributions of foreground stars (MacGillivray \& Stobie 1984). To estimate the extent to which stars have been misclassified by the APM software, we have examined deep CCD images of the regions $\approx 6^{\prime}$ from the 
cores of NGC 7089 and NGC 288. These clusters both show significant departures from King-like surface density profiles, and the seeing conditions under which the plates were taken were average, and slightly worse than average, respectively. Images fainter than APM $B_{J}=20$ were inspected visually and were classified as galaxies if they appeared to be significantly out of round or underluminous in their cores. Of 42 images near NGC 288 classified by the APM as stellar, we found 6 which satisfied our galaxy criteria. 1 out of 7 images classified as non-stellar appear stellar in the CCD image. In NGC 7089, 11 out of 53 images classified by the APM as stellar have profiles which appear galactic in character. Conversely, 5 out of 12 images classified as non-stellar are quite stellar in appearance in our CCD images. The conclusions we draw are that $(i)$ some image confusion is indeed present at faint magnitude levels, and $(i i)$ stars and galaxies are misclassified with approximately equal frequencies, with a possible bias favoring galaxies.

In Figures 11 and 12 are shown the radial profiles of images classified as non-stellar for NGC 288 and NGC 7089. The power-law profiles beyond $r_{t}$ apparent in the (predominantly) stellar samples are not in evidence among the galaxy counts. Indeed, the non-stellar image densities appear to some extent to be anti-correlated with the total image counts, particularly where the uncertainties in the star counts are relatively small.

The clustering properties of galaxies are reasonably well known (Peebles \& Hauser 1974; Heydon-Dumbleton et al. 1988). If the contours in Figure 13 are significantly affected by misclassified galaxies, then we should see the corresponding signature in the two-point correlation function, $\omega(\theta)$. In Figure 15 we show the run of the ensemble estimator (Hewett 1982)

$$
q \hat{\omega}(\theta)=\frac{\left\langle N_{i} N_{j}\right\rangle}{\left\langle N_{i}\right\rangle\left\langle N_{j}\right\rangle}-1
$$

for all images (i.e. of all colors and magnitudes) surrounding NGC 7089 and classified as stellar and non-stellar, respectively. The surface densities $N_{i}, N_{j}$ are computed within cells of dimensions $3.1^{\prime} \times 3.1^{\prime}$ and foreground-subtracted with a $1 \times 1$ bivariate polynomial (see Figure (1) to remove the field-star gradient. In computing $\hat{\omega}$ we avoid the rectangular region extending from $40^{\prime}$ south to $55^{\prime}$ north of the cluster wherein the majority of postulated extra-tidal stars reside. The galaxies show the expected power-law form, with an apparent break occurring near $0.5^{\circ}$. This is somewhat smaller than that found in galaxy surveys and is most likely due to the depth and incompleteness of our counts. The run of $\hat{\omega}$ for the stellar images is gratifyingly close to zero on all scales, which is what one would expect for a purely Poissonian distribution. This is also an indication that our counts do not suffer unduly from plate or machine measuring effects. 
In panels $e$ of Figure 13 we show the smoothed distributions of images classified as non-stellar, after removal of a surface fit of the same order as that used for the stars. The contours levels are numerically identical to the contour levels used in panels $a$. The $1^{\circ}$ structure expected from galaxy counts is apparent, but there is little detailed correspondence between this and the structure apparent in panels $a$. Indicated on these maps are the linear correlation coefficients, $\xi$, determined by comparing the sky-subtracted surface densities of stellar and non-stellar images in bins $25^{\prime}$ square. We have included in these calculations only the area of the scan beyond the $30^{\prime}$ so as not to be influenced by variable crowding effects. NGC 362, NGC 2808, and NGC 3201 show the highest correlations. These are due to image classification uncertainties induced by the high surface densities and to differential obscuration by disk material. Aside from these clusters, NGC 7089 shows the highest correlation coefficient (0.17). In Figure 10 we compare directly the sky-subtracted surface densities of stellar and non-stellar images in the field surrounding NGC 7089. The strength of the correlation apparently relies most heavily on a few outlying points and appears to be insignificant otherwise. The probability that 60 measurements of two uncorrelated variables would give a correlation coefficient as large as 0.17 is $\approx 21 \%$. We conclude from this and the above tests, apart from the tree clusters mentioned above, that the contours in panels $a$ are not significantly affected by misclassification of images.

The question of obscuration is a more difficult one to address. In the absence of image misclassification, we could in principle determine the extent of the obscuration from the variation in galaxy counts across the field. However, given the effects of image crowding on misclassification of images and the likelihood that the obscuring material is not confined to the region between us and the cluster in question, any such attempt would be fraught with uncertainties. We simply note that the surface density contours surrounding NGC 2808 and NGC 3201 may be significantly affected by obscuration, as indicated by the relatively high correlations between the densities of stellar and non-stellar images. The contours in Figure 13 for these clusters should be interpreted with this in mind.

\section{Discussion}

As noted above, the extra-tidal extensions in Figure 6 bear a striking resemblance to the halos and associated tidal tails discussed by Oh and Lin (1992) and Grillmair et al. (1995). The extensions become apparent at a surface density between 4 and 6 orders of magnitude below the central surface density, consistent with the N-body simulations. In Figure 17 we compare the surface density profiles of NGC 7089 and Grillmair et al. 's model 
64eoa near its 14th perigalacticon. This model cluster was evolved along an eccentric orbit with perigalactic distance of $2.5 \mathrm{kpc}$ and apogalactic distance of $10 \mathrm{kpc}$ in a logarithmic potential with circular velocity of $220 \mathrm{~km} \mathrm{~s}^{-1}$. The model units have been scaled so that $r_{h}=10 p c$ and the cluster appears as it would from a distance of $12 \mathrm{kpc}$ (Peterson 1993). The viewing angle is chosen to be along the line connecting the cluster and the galactic center. Grillmair et al. (1995) used a Jaffe model as their starting point in the simulations, giving rise to the evidently non-King-like behavior of the surface density profile in Figure 17. Nonetheless, the agreement between the form of the model profile and the data beyond $r_{t}$ is intriguing.

Grillmair et al. (1995) find that both the amplitude and the slope of the surface density profile beyond the tidal radius are a function of the shape of the cluster orbit, the orbital phase of the cluster, and the viewing angle. There are too many parameters available to put meaningful constraints on the possible orientation and orbital phase of NGC 7089. We simply note that the length scales and density contrasts apparent in the observations are consistent with results of numerical simulations. Extra-tidal stars have been found in dwarf spheroidal satellites of the Milky Way Galaxy (Irwin \& Hatzidimitriou 1993). That tidal tails in the near vicinity of our sample clusters should still be detectable at the present epoch is presumably a consequence of continual tidal shocking, disk shocking, tidal torquing, and ongoing 2-body encounters in the cores. The diversity in the radial gradients of the extra-tidal material among the clusters we have examined is consistent with the orbital shape and phase dependence revealed in the numerical simulations.

In Figure 18 we show contour plots of the two-dimensional surface density distribution of Grillmair et al. 's (1995) model 64eoa at its sixth orbital apocenter as it would appear from a distance of $12 \mathrm{kpc}$ from three different perspectives. The surface densities have been smoothed with the same kernel and masking radius used for NGC 7089 in Figure 13. The forms of the surface density distributions are consistent to a greater or lesser extent with many of the contour maps in Figure 13. In the case of NGC 7089, Figure 13 indicates that the absolute proper motion is directed almost perpendicularly to the apparent major axis of the extra-tidal material. Though this might seem surprising, inspection of Figure 18 suggests that we may be seeing NGC 7089 near it's apogalacticon and that the angle between the plane of the cluster's orbit and our line of sight is relatively large. The latter would be consistent with the Galactic coordinates and derived space motion of this cluster (Cudworth \& Hanson 1993). The magnitude of the derived space motion $\left(\approx 300 \mathrm{~km} \mathrm{~s}^{-1}\right.$ in the Galactic rest frame) is rather large, even if the cluster is assumed to be on a circular orbit, though the uncertainties are substantial. The space motion derived for NGC 7078 indicates that it's orbital plane is more highly inclined to our line of sight, consistent with the more "in-line" appearance of it's extra-tidal material. For the remaining clusters, 
the lack of velocity information and the poor statistics of the observed surface density distributions conspire to make distinctions concerning probable viewing angles largely meaningless.

We conclude that the stars we find beyond the best-fit values of $r_{t}$ are probably unbound as a result of previous and ongoing stripping episodes. Given that our highest quality data show quite pronounced extra-tidal extensions, and that the majority of clusters in our sample appear to have at least some extra-tidal material, we speculate that globular clusters in general have no observable limiting radii. Without a priori knowledge of the spatial distribution of unbound stars in the vicinity of a cluster, the limits imposed by the Galactic tidal field on the spatial distribution of bound stars can only be estimated by model-dependent means. If the underlying distribution of bound stars is King-like, then the tidal cutoff in the binding energy can best be determined by fitting models only to data not significantly affected by the presence of unbound stars. In effect, the presence of unbound stars places an upper limit on the accuracy to which we can resolve the tidal radii of globular clusters. Despite the quality of our data, our ability to determine the distribution of globular cluster orbit shapes (or conversely, the Galactic mass distribution) is little better than that of previous investigators (Peterson 1974; Innanen, Harris, \& Webbink 1983).

Some of the smoothed surface density contour maps in Figure 13 are suggestive of tidal tails extending to considerable distances from the clusters. To verify that these tails are physically associated with the clusters will require spectroscopy of a reasonable sample of extra-tidal stars with colors and magnitudes consistent with those of the clusters. If their velocities and metallicities are similar to those of bound cluster stars, we may be reasonably confident that the tails are not due to other stellar associations in the field. Sufficient velocity resolution would be required to distinguish between cluster and halo stars. On the other hand, the radial velocities of the extra-tidal stars at some distance from the cluster may be expected to differ appreciably from that of the cluster itself, depending on the shape of the cluster's orbit, the orbital phase of the cluster, and the orientation with respect to our line of sight. As an example, we show in Figure 19 the line-of-sight velocities for particles in the model used to construct Figure 18. The velocities shown have been projected along the line connecting the cluster to the galactic center, after subtracting the motion of the cluster itself. While the velocity variations across the field may or may not be large depending on the variables listed above, the velocity dispersions of the stars at any point within the tidal tails will be comparatively small. Since most of our observed extra-tidal stars have $B_{J}>20$ and the numbers of tail stars are not large to begin with, a program to spectroscopically confirm the association of these stars with their parent clusters would entail considerable effort, presumably requiring multi-object spectrographs on 4-meter-class telescopes. 
Given the great ages of globular clusters, we expect that the tidal tails we have found should extend well beyond the $4^{\circ}$ fields we have examined. For NGC 7089 we find that the one-dimensional surface density profile beyond $r_{t}$ goes as $f \propto r^{-2}$, implying that the two-dimensional surface density is proportional to $r^{-1}$. It may be possible to detect unbound cluster stars at even larger distances from the cluster than we have examined here. The appearance of the tidal tails on large scales is determined primarily by the cluster's orbit shape and less by the orbital phase of the cluster or the details of the stripping process. Defining the large-scale distribution of extra-tidal stars on the sky would, in principle, allow us to actually trace the cluster's orbit. Estimates of the tidal radii of globular clusters may not be as useful for studying Galactic dynamics as we had hoped, but it may yet be possible to determine orbit shapes of clusters from the distributions and velocities of their leavings.

We thank the staff of the United Kingdom Schmidt Telescope Unit for their untiring efforts to acquire the plate material used in this investigation. CJG acknowledges the support of an Australian National University PhD scholarship. 


\section{REFERENCES}

Bahcall, J.N., \& Soneira, R.M. 1980, ApJS, 44, 73

Binney, J., \& Tremaine, S. 1987, Galactic Dynamics, Princeton University Press, Princeton.

Bunclark, P.S., \& Irwin, M.J. 1983, in Proceedings of Statistical Methods in Astronomy, p. 195, E.J. Roffe (ed.), ESA SP-201.

Chun, M.S., \& Freeman, K.C. 1979, ApJ, 227, 93

Cudworth, K.M., \& Hanson, R.B. 1993, AJ, 105, 168

Djorgovski, S. 1993, in Structure and Dynamics of Globular Clusters, p. 373, S.Djorgovski \& G. Meylan (eds.), ASPCS vol. 50

Djorgovski, S., \& King, I.R. 1984, ApJ, 277, L49

Faulkner, D.J., Scott, T.R., Wood, P.R., \& Wright, A.E. 1991, ApJ, 274, L45

Freeman, K.C., \& Norris, J. 1981, ARA\&A, 19, 319

Grillmair, C.J., Quinn, P.J., Freeman, K.C., Salmon, J. 1995, in preparation.

Grindlay, J.E., Hertz, P., Steiner, J.E., Merray, S.S., \& Lightman, A.P. 1984, ApJ, 282, L13

Hanes, D.A., \& Brodie, J.P. 1985, MNRAS, 214, 491

Hewett, P.C. 1982, MNRAS, 201, 867

Heydon-Dumbleton, N.H., Collins, C.A., \& MacGillivray, H.T. 1988, in Large Scale Structures in the Universe: Observational and Analytical Methods, W.C. Seitter, H.W. Duerbeck, and M. Tacke (eds.), Springer-Verlag, Berlin.

Illingworth, G.D., \& Illingworth, W. 1976, ApJS, 30, 227

Innanen, K.A., Harris, W.E., \& Webbink, R.F. 1983, AJ, 88, 338

Irwin, M.J. 1985, MNRAS, 214, 575

Irwin, M.J., \& Trimble, V. 1984, AJ, 89, 83

Irwin, M.J., Demers, S., \& Kunkel, W.E. 1990, å, 99, 191

Johnson, H.L. 1959, Lowell Obs. Bull. 4, 117 
Keenan, D.W. 1981, å, 95, 340

Kibblewhite, E.J., Bridgeland, M.T., Bunclark, P.S., \& Irwin, M.J. 1984, in Astronomical Microdensitometry Conference NASA-2317, p. 277, D.A. Klinglesmith (ed.), NASA, Washington.

King, I.R. 1962, AJ, 67, 471

King, I.R. 1966, AJ, 71, 64

King, I.R. 1988, PASP, 100, 999

King, I.R., Hedemann, E., Hodge, S.M., \& White, R.E. 1968 AJ, 73, 456

Kron, G.E., \& Mayall, N.U. 1960, AJ, 65, 58

Kron, G.E., Hewitt, A.V., \& Wasserman, L.H. 1984, PASP, 96, 198

Kron, G.E. \& Gordon, K.C. 1986, PASP, 98, 98

Lauer, T.R., Holtzman, J.A., Faber, S.M., Baum, W.A., Currie, D.G., Ewald, S.P., Groth, E.J., Hester, J.J., Kelsall, T., Light, R.M., Lynds, C.R., O'Neil, E.J., Schneider, D.P., Shaya, E.J., Westphal, J.A. 1991, ApJ, 369, L45

MacGillivray, H.T., \& Stobie, R.S. 1984, Vistas Astron., 27, 433

Mamon, G.A., \& Soneira, R.M. 1982, ApJ, 255, 181

Oh, K.S., \& Lin, D.N.C. 1992, ApJ, 386, 519

Peebles, P.J.E., \& Hauser, M.G. 1974, ApJS, 28, 19

Peterson, C.J. 1974, ApJ, 190, L17

Peterson, C.J. 1976, AJ, 81, 617

Peterson, C.J. 1986a, Astronomical Data Center Bulletin.

Peterson, C.J. 1986b, PASP, 98, 192

Peterson, C.J. 1993, in Structure and Dynamics of Globular Clusters, p. 337, S.Djorgovski \& G. Meylan (eds.), ASPCS vol. 50

Peterson, C.J., \& King, I.R. 1975, AJ, 80, 427

Seitzer, P.O. 1983, PhD Thesis, University of Virginia. 
Shapley, H. 1918, PASP, 30, 42

Shawl, S.J., \& White, R.E. 1986, AJ, 91, 312

Trager, S. Djorgovski, S., \& King, I.R. 1993, in Structure and Dynamics of Globular Clusters, p. 347, S.Djorgovski \& G. Meylan (eds.), ASPCS vol. 50

von Hoerner, S. 1957, ApJ, 125, 451 
Fig. 1.- APM color-magnitude diagrams of all images labeled as stars for each of our clusters. The left-hand panels show the color-magnitude distribution of stars lying typically between 0.1 and $0.5 r_{t}$, and the right-hand panels show the distribution of field stars. The heavy lines indicate the regions chosen for counting purposes to give the highest signal-tonoise ratios in the outskirts of each cluster.

Fig. 2.- Contour plot of the smoothed density distribution of cluster stars in NGC 3201. The thin lines map the density distribution of cluster stars as per Equation 1. The heavy line is a contour of constant signal-to-noise ratio (computed using Equation 2 and subsequently smoothed) arbitrarily chosen to match the density distribution on the red side of the cluster sequence.

Fig. 3.- Cumulative star-count signal-to-noise ratio $S$ as a function of enclosed colormagnitude area, $a_{k}$, for NGC 362 . 
Fig. 4.- Comparison of 1st and 3rd order bivariate polynomial fits to the foreground distribution surrounding NGC 7089. The regions excluded from the surface fit are indicated by heavy lines.

Fig. 5.- The software réseau used for the star counts in Tables 3 through 14. Sector numbers are indicated.

Fig. 6.- Surface density profiles of our sample clusters. Hollow symbols refer to aperture photometry, filled symbols represent crowding-corrected star count data, and the $\times$ s indicate our star counts uncorrected for crowding. References to the original sources of data are given in Table 15. The solid lines are King models, normalized to our star-count data, with core and tidal radii taken from Table 1 . The run of field star surface densities (corrected for crowding effects) are shown by dotted lines.

Fig. 7.- Ratio of bright stars to faint stars as a function of radius in a representative selection of sample clusters.

Fig. 8.- Ratio of bright stars to faint stars as a function of total surface density in a representative selection of sample clusters.

Fig. 9.- Contributions to the total surface density by different classes of images. $\otimes \mathrm{s}$ represent images "deemed" merged and include the excess surface density of non-stellar images at small radii. The curve shows the expected density of merged objects based on Equation 7 and an image area of $34 \operatorname{arcsec}^{2}$.

Fig. 10.- Residuals after subtracting (in the log domain) normalized King models from the surface density data. Arrows indicate core and tidal radii from Table 1. References to sources of data are given in Table 15.

Fig. 11. - Surface density profiles for NGC 288 computed using both $1 \times 1$ and $3 \times 3$ fits to the surface densities of foreground stars. Also shown are the surface densities computed for objects classified as non-stellar, using a $1 \times 1$ fit to the distribution of field objects. The solid curve is a King model with core and tidal radii from Table 1.

Fig. 12.- Same as Figure 11, but for NGC 7089. 
Fig. 13. - Contour maps of the background-subtracted and Fourier-smoothed surface density distributions. Panels $a$ show the contours of images classified as stars after Fourier smoothing with a Gaussian kernel of $16^{\prime}$. The inner regions of the clusters have been cosbelled out to reveal structure at large radii. Boxed numbers indicate deviations from zero in units of the standard deviation, and the heavy, circular contours arise from smoothing King-model surface density distributions in an identical manner. The numerical values of these heavy contours are identical to the lowest (dashed) contours of the data. Arrows at the centers of the clusters indicate the direction to the Galactic center. Where more than one arrow is shown, the longer of the two indicates the direction of the measured, absolute proper motion of the cluster. Panels $b$ and $c$ show the results of 1000 Poissonian realizations of each field, as described in the text. For those cases in which excess power on large scales is evident, panels $d$ show the smoothed contours of just the lowest three wavenumbers with power amplitudes significant at $>99 \%$ confidence level. Panels $e$ show similarly smoothed contours of the surface density distribution of images classified as non-stellar. For each cluster, the lowest (dashed) contours and the contour intervals in panels $a, d$, and $e$ are identical.

Fig. 14. - Color-magnitude distribution of images extending westwards from NGC 6864.

Fig. 15. - Two-point correlation function for stellar (filled circles) and non-stellar (open circles) images surrounding NGC 7089. Error bars are shown for alternating points to avoid confusion.

Fig. 16. - Comparison of the foreground-subtracted surface densities of non-stellar images with those of stellar images for the field surrounding NGC 7089. 
Fig. 17. - Comparison between a subset of stars counts and aperture photometry for NGC 7089 with the surface density profile of model 64eoa of Grillmair et al. (1995) after 14 orbits. The solid line corresponds to a King model with core and tidal radii taken from Table 1, and the dashed line shows the surface density profile of our model as it would appear from a distance of $12 \mathrm{kpc}$ looking out from the galactic center. Codes for data sources are given in Table 15.

Fig. 18. - Smoothed, 2-dimensional surface density distribution of particles in model 64eoa of Grillmair et al. (1995) after six perigalactic passages, as viewed from a distance of $12 \mathrm{kpc}$ along a line connecting the cluster and the Galactic center (top), along a tangent to the orbital path (middle), and along a line perpendicular to the orbital plane (bottom). The cluster's direction of motion is indicated in each case.

Fig. 19.- Particle velocities, projected along a line connecting the cluster and the galactic center, for the model shown smoothed in Figure 18. The model used is near it's orbital apogalacticon so that the tidal tails extend nearly perpendicularly to the line of sight. The projected distances from the cluster center have been computed assuming a distance of 12 kpc between the cluster and the observer. 
TABle 1. Relevant Cluster Data.

\begin{tabular}{|c|c|c|c|c|c|c|c|}
\hline Cluster & $\begin{array}{c}\alpha^{\mathrm{a}} \\
(1950)\end{array}$ & $\begin{array}{c}\delta^{\mathrm{a}} \\
(1950)\end{array}$ & $\begin{array}{l}l^{I I} \\
\left(^{\circ}\right)\end{array}$ & $\begin{array}{l}b^{I I} \\
\left({ }^{\circ}\right)\end{array}$ & $\begin{array}{c}r_{c}^{\mathrm{b}} \\
(\operatorname{arcmin})\end{array}$ & $\begin{array}{c}r_{t}^{\mathrm{b}} \\
(\operatorname{arcmin})\end{array}$ & $\begin{array}{l}R_{G C}{ }^{\mathrm{c}} \\
(\mathrm{kpc})\end{array}$ \\
\hline NGC 288 & 005021 & -265141 & 152.2 & -89.4 & 1.42 & 12.9 & 11.7 \\
\hline NGC 362 & 010133 & -710659 & 301.5 & -46.2 & 0.17 & 14.9 & 9.3 \\
\hline NGC 1904 & 052207 & -243408 & 227.2 & -29.4 & 0.16 & 8.4 & 19.1 \\
\hline NGC 2808 & 091104 & -643923 & 282.2 & -11.3 & 0.26 & 15.6 & 11.0 \\
\hline NGC 3201 & 101534 & -460938 & 227.2 & 8.6 & 1.45 & 29.6 & 8.9 \\
\hline NGC 4590 & 123649 & -262806 & 299.6 & 36.1 & 0.69 & 30.3 & 9.6 \\
\hline NGC 5824 & 150054 & -325223 & 332.6 & 22.1 & 0.06 & 15.6 & 26.3 \\
\hline NGC 6864 & 200308 & -220355 & 20.3 & -25.7 & 0.10 & 7.3 & 12.6 \\
\hline NGC 6934 & 203144 & 071355 & 52.1 & -18.9 & 0.25 & 8.4 & 12.0 \\
\hline NGC 6981 & 205043 & -124337 & 35.2 & -32.7 & 0.54 & 9.2 & 13.8 \\
\hline NGC 7078 & 212733 & 115649 & 65.0 & -27.3 & 0.07 & 21.5 & 10.5 \\
\hline NGC 7089 & 213055 & -010244 & 53.4 & -35.8 & 0.34 & 21.5 & 10.7 \\
\hline
\end{tabular}

Notes to Table 1.

aShawl \& White (1986).

bTrager, Djorgovski, \& King (1993).

${ }^{\mathrm{c}}$ Djorgovski (1993). 
TABle 2. Observations.

\begin{tabular}{|c|c|c|c|c|c|}
\hline Cluster & Plate ID\# & Emulsion & Filter & $\begin{array}{l}\text { Exposure Time } \\
\text { minutes }\end{array}$ & $\begin{array}{l}\text { Image Diameter } \\
\text { microns }\end{array}$ \\
\hline \multirow[t]{4}{*}{ NGC 288} & 13390 & IIIaF & RG 630 & 105 & 50 \\
\hline & 13437 & IIIaF & RG 630 & 90 & 40 \\
\hline & 13840 & IIIaJ & GG 395 & 65 & 45 \\
\hline & 13850 & IIIaJ & GG 395 & 65 & 35 \\
\hline \multirow[t]{4}{*}{ NGC 362} & 13276 & IIIaF & RG 630 & 45 & 25 \\
\hline & 13277 & IIIaJ & GG 395 & 45 & 45 \\
\hline & 14000 & IIIaF & RG 630 & 45 & 35 \\
\hline & 13999 & IIIaJ & GG 395 & 30 & 35 \\
\hline \multirow[t]{4}{*}{ NGC 1904} & 13421 & IIIaJ & GG 395 & 65 & 35 \\
\hline & 13432 & IIIaJ & GG 395 & 70 & 30 \\
\hline & 13453 & IIIaF & RG 630 & 90 & 35 \\
\hline & 13455 & IIIaF & RG 630 & 85 & 50 \\
\hline \multirow[t]{4}{*}{ NGC 2808} & 13482 & IIIaJ & GG 395 & 25 & 25 \\
\hline & 13509 & IIIaF & RG 630 & 35 & 35 \\
\hline & 13542 & IIIaJ & GG 395 & 25 & 50 \\
\hline & 13543 & IIIaF & RG 630 & 35 & 35 \\
\hline \multirow[t]{4}{*}{ NGC 3201} & 13549 & IIIaJ & GG 395 & 25 & 30 \\
\hline & 13550 & IIIaF & RG 630 & 35 & 35 \\
\hline & 13561 & IIIaJ & GG 395 & 25 & 30 \\
\hline & 13562 & IIIaF & RG 630 & 35 & 35 \\
\hline \multirow[t]{4}{*}{ NGC 4590} & 13606 & IIIaF & RG 630 & 90 & 40 \\
\hline & 13737 & IIIaJ & GG 395 & 60 & 35 \\
\hline & 13754 & IIIaF & RG 630 & 90 & 50 \\
\hline & 13756 & IIIaJ & GG 395 & 60 & 35 \\
\hline \multirow[t]{4}{*}{ NGC 5824} & 13615 & IIIaJ & GG 395 & 65 & 35 \\
\hline & 13621 & IIIaJ & GG 395 & 60 & 40 \\
\hline & 13666 & IIIaF & RG 630 & 90 & 30 \\
\hline & 13673 & IIIaF & RG 630 & 90 & 35 \\
\hline \multirow[t]{4}{*}{ NGC 6864} & 13231 & IIIaJ & GG 395 & 25 & 40 \\
\hline & 13240 & IIIaF & RG 630 & 35 & 40 \\
\hline & 13674 & IIIaJ & GG 395 & 25 & 35 \\
\hline & 13654 & IIIaF & RG 630 & 45 & 45 \\
\hline \multirow[t]{3}{*}{ NGC 6934} & 13725 & IIIaJ & GG 395 & 60 & 35 \\
\hline & 13813 & IIIaF & RG 630 & 90 & 35 \\
\hline & 13818 & IIIaJ & GG 395 & 70 & 35 \\
\hline \multirow[t]{4}{*}{ NGC 6981} & 13248 & IIIaF & RG 630 & 120 & 35 \\
\hline & 13274 & IIIaJ & GG 395 & 65 & 35 \\
\hline & 13387 & IIIaF & RG 630 & 105 & 35 \\
\hline & 13829 & IIIaJ & GG 395 & 65 & 40 \\
\hline \multirow[t]{2}{*}{ NGC 7078} & 13339 & IIIaF & RG 630 & 100 & 60 \\
\hline & 13353 & IIIaJ & GG 395 & 70 & 50 \\
\hline
\end{tabular}


TABle 2. (continued)

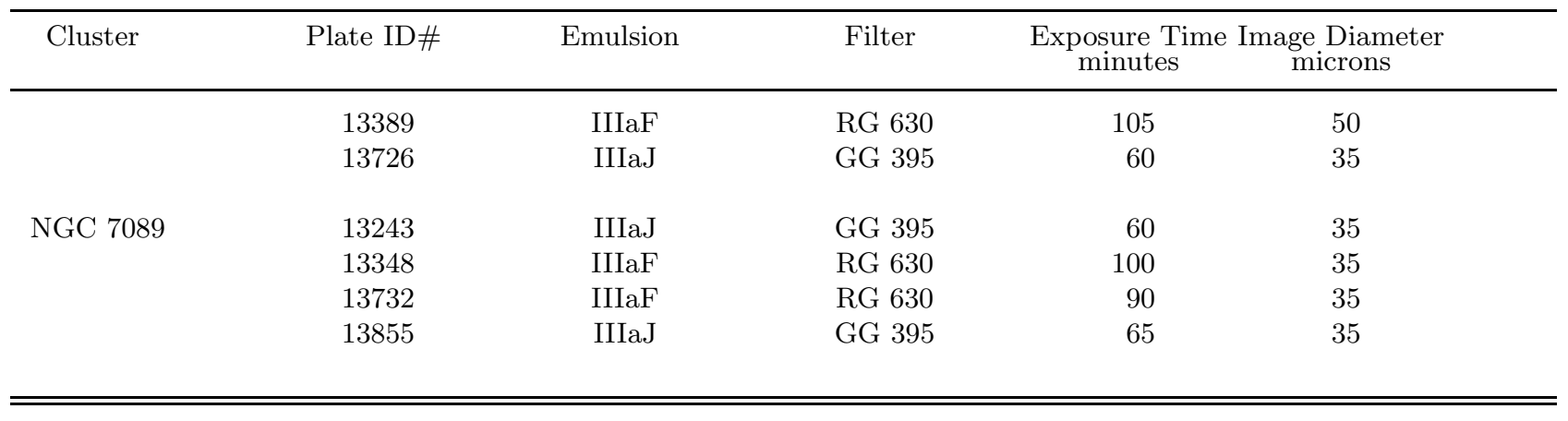


TABle 3. Observations.

\begin{tabular}{|c|c|c|c|c|c|}
\hline Cluster & Plate ID\# & Emulsion & Filter & $\begin{array}{l}\text { Exposure Time } \\
\text { minutes }\end{array}$ & $\begin{array}{l}\text { Image Diameter } \\
\text { microns }\end{array}$ \\
\hline \multirow[t]{4}{*}{ NGC 288} & 13390 & IIIaF & RG 630 & 105 & 50 \\
\hline & 13437 & IIIaF & RG 630 & 90 & 40 \\
\hline & 13840 & IIIaJ & GG 395 & 65 & 45 \\
\hline & 13850 & IIIaJ & GG 395 & 65 & 35 \\
\hline \multirow[t]{4}{*}{ NGC 362} & 13276 & IIIaF & RG 630 & 45 & 25 \\
\hline & 13277 & IIIaJ & GG 395 & 45 & 45 \\
\hline & 14000 & IIIaF & RG 630 & 45 & 35 \\
\hline & 13999 & IIIaJ & GG 395 & 30 & 35 \\
\hline \multirow[t]{4}{*}{ NGC 1904} & 13421 & IIIaJ & GG 395 & 65 & 35 \\
\hline & 13432 & IIIaJ & GG 395 & 70 & 30 \\
\hline & 13453 & IIIaF & RG 630 & 90 & 35 \\
\hline & 13455 & IIIaF & RG 630 & 85 & 50 \\
\hline \multirow[t]{4}{*}{ NGC 2808} & 13482 & IIIaJ & GG 395 & 25 & 25 \\
\hline & 13509 & IIIaF & RG 630 & 35 & 35 \\
\hline & 13542 & IIIaJ & GG 395 & 25 & 50 \\
\hline & 13543 & IIIaF & RG 630 & 35 & 35 \\
\hline \multirow[t]{4}{*}{ NGC 3201} & 13549 & IIIaJ & GG 395 & 25 & 30 \\
\hline & 13550 & IIIaF & RG 630 & 35 & 35 \\
\hline & 13561 & IIIaJ & GG 395 & 25 & 30 \\
\hline & 13562 & IIIaF & RG 630 & 35 & 35 \\
\hline \multirow[t]{4}{*}{ NGC 4590} & 13606 & IIIaF & RG 630 & 90 & 40 \\
\hline & 13737 & IIIaJ & GG 395 & 60 & 35 \\
\hline & 13754 & IIIaF & RG 630 & 90 & 50 \\
\hline & 13756 & IIIaJ & GG 395 & 60 & 35 \\
\hline \multirow[t]{4}{*}{ NGC 5824} & 13615 & IIIaJ & GG 395 & 65 & 35 \\
\hline & 13621 & IIIaJ & GG 395 & 60 & 40 \\
\hline & 13666 & IIIaF & RG 630 & 90 & 30 \\
\hline & 13673 & IIIaF & RG 630 & 90 & 35 \\
\hline \multirow[t]{4}{*}{ NGC 6864} & 13231 & IIIaJ & GG 395 & 25 & 40 \\
\hline & 13240 & IIIaF & RG 630 & 35 & 40 \\
\hline & 13674 & IIIaJ & GG 395 & 25 & 35 \\
\hline & 13654 & IIIaF & RG 630 & 45 & 45 \\
\hline \multirow[t]{3}{*}{ NGC 6934} & 13725 & IIIaJ & GG 395 & 60 & 35 \\
\hline & 13813 & IIIaF & RG 630 & 90 & 35 \\
\hline & 13818 & IIIaJ & GG 395 & 70 & 35 \\
\hline \multirow[t]{4}{*}{ NGC 6981} & 13248 & IIIaF & RG 630 & 120 & 35 \\
\hline & 13274 & IIIaJ & GG 395 & 65 & 35 \\
\hline & 13387 & IIIaF & RG 630 & 105 & 35 \\
\hline & 13829 & IIIaJ & GG 395 & 65 & 40 \\
\hline \multirow[t]{2}{*}{ NGC 7078} & 13339 & IIIaF & RG 630 & 100 & 60 \\
\hline & 13353 & IIIaJ & GG 395 & 70 & 50 \\
\hline
\end{tabular}


TABLe 3. (continued)

\begin{tabular}{|c|c|c|c|c|c|}
\hline Cluster & Plate ID\# & Emulsion & Filter & $\begin{array}{l}\text { Exposure Time } \\
\text { minutes }\end{array}$ & $\underset{\text { microns }}{\text { Image Diameter }}$ \\
\hline \multirow{6}{*}{ NGC 7089} & 13389 & IIIaF & RG 630 & 105 & 50 \\
\hline & 13726 & IIIaJ & GG 395 & 60 & 35 \\
\hline & 13243 & IIIaJ & GG 395 & 60 & 35 \\
\hline & 13348 & IIIaF & RG 630 & 100 & 35 \\
\hline & 13732 & IIIaF & RG 630 & 90 & 35 \\
\hline & 13855 & IIIaJ & GG 395 & 65 & 35 \\
\hline
\end{tabular}


TABle 4. NGC 288 Star Counts.

\begin{tabular}{|c|c|c|c|c|c|c|}
\hline$r$ & $r_{e f f}$ & $\mathrm{~N}$ & $\mathrm{f}_{b k d}$ & $\alpha_{c}$ & $\mathrm{f}$ & Error \\
\hline $0-1$ & 0.71 & 17 & 0.152 & - & - & - \\
\hline $1-2$ & 1.58 & 86 & & - & - & - \\
\hline $2-3$ & 2.55 & 209 & 52 & - & - & - \\
\hline $3-4$ & 3.54 & 401 & 52 & 3.923 & 71.381 & 3.572 \\
\hline $4-5$ & 4.53 & 463 & 52 & 1.705 & 27.774 & 1.298 \\
\hline $5-6$ & 5.52 & 400 & 52 & .341 & 15.373 & 0.776 \\
\hline $6-7$ & 6.52 & 291 & 52 & .172 & 8.196 & 0.489 \\
\hline $7-8$ & 7.52 & 229 & 2 & 3 & .256 & 0.357 \\
\hline $8-9$ & 8.5 & 141 & 2 & & 56 & 0.236 \\
\hline $9-10$ & 9. & 96 & 2 & & 17 & 0.170 \\
\hline $10-12$ & 11.0 & 100 & 2 & 3 & 88 & 0.074 \\
\hline $12-14$ & 1 & 78 & 2 & 0 & 35 & 0.055 \\
\hline $14-16$ & $1:$ & 44 & 2 & 4 & 0.085 & 0.036 \\
\hline $16-18$ & 17.0 & 42 & 2 & 1. & 0.047 & 0.031 \\
\hline $18-20$ & 19.03 & 49 & 2 & $1 .($ & 0.056 & 0.030 \\
\hline $20-25$ & 22.64 & 118 & .1 & $1 .($ & 0.017 & 0.016 \\
\hline $25-30$ & 27.61 & 134 & 0.152 & 1.015 & 0.005 & 0.014 \\
\hline $30-35$ & 32.60 & 182 & 0.152 & 1.0 & 0.029 & 0.013 \\
\hline $35-40$ & 37.58 & 186 & 0.152 & 1.017 & 0.008 & 0.012 \\
\hline $40-50$ & 45.28 & 477 & 0.152 & 1.017 & 0.019 & 0.008 \\
\hline $50-60$ & 55.23 & 531 & 0.152 & 1.017 & 0.004 & 0.007 \\
\hline $60-70$ & 65.19 & 607 & 0.152 & 1.016 & -0.001 & 0.006 \\
\hline $70-80$ & 75.17 & 717 & 0.152 & 1.017 & 0.003 & 0.006 \\
\hline $80-$ & 85.15 & 814 & 0.152 & 1.016 & 0.003 & 0.005 \\
\hline $90-100$ & 95.13 & 884 & 0.152 & 1.016 & -0.002 & 0.005 \\
\hline & \multirow{2}{*}{\multicolumn{3}{|c|}{$\begin{array}{l}\text { Sectors used: } \\
\text { Surface Fit Order: }\end{array}$}} & \multicolumn{3}{|l|}{$1-\varepsilon$} \\
\hline & & & & $1 \times$ & & \\
\hline & \multicolumn{3}{|c|}{ Image Area $A$ : } & \multicolumn{3}{|c|}{$27.2 \operatorname{arcsec}^{2}$} \\
\hline
\end{tabular}


TABle 5. NGC 362 Star Counts.

\begin{tabular}{crrrrrr}
\hline \hline$r$ & $r_{\text {eff }}$ & \multicolumn{1}{c}{$\mathrm{N}$} & $\mathrm{f}_{b k d}$ & $\alpha_{c}$ & $\mathrm{f}$ & Error \\
\hline $0-1$ & 0.71 & 0 & 0.848 & - & - & - \\
$1-2$ & 1.58 & 20 & 0.841 & - & - & - \\
$2-3$ & 2.55 & 139 & 0.829 & - & - & - \\
$3-4$ & 3.54 & 281 & 0.816 & - & - & - \\
$4-5$ & 4.53 & 277 & 0.805 & 2.551 & 39.183 & 2.403 \\
$5-6$ & 5.52 & 256 & 0.795 & 1.605 & 18.226 & 1.189 \\
$6-7$ & 6.52 & 162 & 0.784 & 1.261 & 7.218 & 0.629 \\
$7-8$ & 7.52 & 142 & 0.773 & 1.213 & 5.073 & 0.491 \\
$8-9$ & 8.51 & 99 & 0.764 & 1.150 & 2.646 & 0.343 \\
$9-10$ & 9.51 & 73 & 0.754 & 1.120 & 1.438 & 0.257 \\
$10-12$ & 11.05 & 105 & 0.739 & 1.102 & 0.600 & 0.131 \\
$12-14$ & 13.04 & 102 & 0.721 & 1.097 & 0.375 & 0.109 \\
$14-16$ & 15.03 & 112 & 0.704 & 1.087 & 0.330 & 0.098 \\
$16-18$ & 17.03 & 121 & 0.687 & 1.084 & 0.295 & 0.089 \\
$18-20$ & 19.03 & 86 & 0.672 & 1.072 & -0.054 & 0.067 \\
$20-25$ & 22.64 & 270 & 0.647 & 1.076 & 0.011 & 0.040 \\
$25-30$ & 27.61 & 302 & 0.615 & 1.074 & -0.015 & 0.035 \\
$30-35$ & 32.60 & 364 & 0.588 & 1.070 & 0.023 & 0.032 \\
$35-40$ & 37.58 & 377 & 0.563 & 1.067 & -0.017 & 0.028 \\
$40-50$ & 45.28 & 924 & 0.532 & 1.063 & 0.024 & 0.018 \\
$50-60$ & 55.23 & 1008 & 0.494 & 1.061 & 0.001 & 0.016 \\
$60-70$ & 65.19 & 1069 & 0.457 & 1.055 & -0.015 & 0.014 \\
$70-80$ & 75.17 & 1124 & 0.415 & 1.053 & -0.013 & 0.012 \\
$80-90$ & 85.15 & 1176 & 0.361 & 1.049 & 0.009 & 0.011 \\
& \multicolumn{7}{c}{ Sectors used: } & $1-5$ & & \\
& \multicolumn{7}{c}{ Surface Fit Order: } & $3 \times 2$ & & \\
\hline \hline & \multicolumn{7}{c}{ Image Area A: } & 27.2 & arcsec ${ }^{2}$ & \\
\hline
\end{tabular}


Table 6. NGC 1904 Star Counts.

\begin{tabular}{crrrrrr}
\hline \hline$r$ & $r_{\text {eff }}$ & \multicolumn{1}{c}{$\mathrm{N}$} & $\mathrm{f}_{b k d}$ & \multicolumn{1}{c}{$\alpha_{c}$} & $\mathrm{f}$ & Error \\
\hline $0-1$ & 0.71 & 0 & 0.276 & - & - & - \\
$1-2$ & 1.58 & 91 & 0.276 & - & - & - \\
$2-3$ & 2.55 & 345 & 0.276 & 3.069 & 67.128 & 3.629 \\
$3-4$ & 3.54 & 360 & 0.276 & 1.510 & 24.442 & 1.303 \\
$4-5$ & 4.53 & 224 & 0.276 & 1.177 & 9.048 & 0.623 \\
$5-6$ & 5.52 & 129 & 0.276 & 1.090 & 3.794 & 0.358 \\
$6-7$ & 6.52 & 86 & 0.276 & 1.059 & 1.954 & 0.240 \\
$7-8$ & 7.52 & 84 & 0.276 & 1.044 & 1.586 & 0.203 \\
$8-9$ & 8.51 & 46 & 0.276 & 1.036 & 0.616 & 0.132 \\
$9-10$ & 9.51 & 39 & 0.276 & 1.034 & 0.400 & 0.108 \\
$10-12$ & 11.05 & 67 & 0.276 & 1.031 & 0.224 & 0.061 \\
$12-14$ & 13.04 & 73 & 0.276 & 1.030 & 0.185 & 0.054 \\
$14-16$ & 15.03 & 66 & 0.276 & 1.026 & 0.084 & 0.044 \\
$16-18$ & 17.03 & 78 & 0.276 & 1.029 & 0.100 & 0.043 \\
$18-20$ & 19.03 & 72 & 0.276 & 1.030 & 0.035 & 0.037 \\
$20-25$ & 22.64 & 222 & 0.276 & 1.028 & 0.047 & 0.022 \\
$25-30$ & 27.61 & 223 & 0.276 & 1.027 & -0.010 & 0.018 \\
$30-35$ & 32.60 & 270 & 0.276 & 1.027 & -0.004 & 0.017 \\
$35-40$ & 37.58 & 288 & 0.276 & 1.025 & -0.025 & 0.015 \\
$40-50$ & 45.28 & 736 & 0.276 & 1.027 & -0.008 & 0.010 \\
$50-60$ & 55.23 & 897 & 0.276 & 1.026 & -0.009 & 0.009 \\
$60-70$ & 65.19 & 1120 & 0.276 & 1.027 & 0.006 & 0.008 \\
$70-80$ & 75.17 & 1287 & 0.276 & 1.027 & 0.005 & 0.008 \\
$80-90$ & 85.15 & 1449 & 0.276 & 1.026 & 0.003 & 0.007 \\
$90-100$ & 95.13 & 1611 & 0.276 & 1.027 & 0.002 & 0.007 \\
& \multicolumn{7}{c}{ Sectors used: } & $1-8$ & & \\
& \multicolumn{7}{c}{ Surface Fit Order: } & $1 \times 1$ & & \\
\hline \hline & \multicolumn{1}{c}{ Image Area A: } & 22.6 arcsec ${ }^{2}$ & \\
\hline
\end{tabular}


TABle 7. NGC 2808 Star Counts.

\begin{tabular}{crrrrrr}
\hline \hline$r$ & \multicolumn{1}{c}{$r_{\text {eff }}$} & \multicolumn{1}{c}{$\mathrm{N}$} & $\mathrm{f}_{b k d}$ & $\alpha_{c}$ & $\mathrm{f}$ & Error \\
\hline $0-1$ & 0.71 & 0 & 3.766 & - & - & - \\
$1-2$ & 1.58 & 9 & 3.766 & - & - & - \\
$2-3$ & 2.55 & 86 & 3.766 & - & - & - \\
$3-4$ & 3.54 & 199 & 3.766 & 6.246 & 52.755 & 4.007 \\
$4-5$ & 4.53 & 271 & 3.766 & 2.574 & 20.906 & 1.499 \\
$5-6$ & 5.52 & 286 & 3.765 & 1.806 & 11.184 & 0.884 \\
$6-7$ & 6.52 & 272 & 3.765 & 1.522 & 6.375 & 0.615 \\
$7-8$ & 7.52 & 234 & 3.765 & 1.354 & 2.957 & 0.439 \\
$8-9$ & 8.51 & 223 & 3.764 & 1.305 & 1.685 & 0.365 \\
$9-10$ & 9.51 & 205 & 3.764 & 1.264 & 0.577 & 0.303 \\
$10-12$ & 11.05 & 482 & 3.763 & 1.271 & 0.668 & 0.202 \\
$12-14$ & 13.04 & 489 & 3.762 & 1.267 & 0.032 & 0.172 \\
$14-16$ & 15.03 & 557 & 3.761 & 1.235 & -0.110 & 0.155 \\
$16-18$ & 17.03 & 671 & 3.759 & 1.251 & 0.171 & 0.152 \\
$18-20$ & 19.03 & 666 & 3.757 & 1.240 & -0.297 & 0.134 \\
$20-25$ & 22.64 & 2154 & 3.754 & 1.250 & 0.057 & 0.082 \\
$25-30$ & 27.61 & 2455 & 3.747 & 1.238 & -0.229 & 0.071 \\
$30-35$ & 32.60 & 3101 & 3.740 & 1.248 & 0.050 & 0.068 \\
$35-40$ & 37.58 & 3457 & 3.731 & 1.243 & -0.083 & 0.062 \\
$40-50$ & 45.28 & 8442 & 3.716 & 1.245 & 0.001 & 0.040 \\
$50-60$ & 55.23 & 10208 & 3.692 & 1.249 & -0.003 & 0.037 \\
$60-70$ & 65.19 & 11974 & 3.664 & 1.250 & 0.001 & 0.033 \\
$70-80$ & 75.17 & 13699 & 3.632 & 1.248 & -0.005 & 0.031 \\
$80-90$ & 85.15 & 15746 & 3.596 & 1.248 & 0.084 & 0.029 \\
$90-100$ & 95.13 & 17165 & 3.557 & 1.239 & 0.005 & 0.027 \\
& & & & & & \\
& & Sectors used: & $1-8$ & & \\
\hline \hline & & Surface Fit Order: & $2 \times 2$ & & \\
\hline
\end{tabular}


Table 8. NGC 3201 Star Counts.

\begin{tabular}{crrrrrr}
\hline \hline$r$ & $r_{\text {eff }}$ & \multicolumn{1}{c}{$\mathrm{N}$} & $\mathrm{f}_{b k d}$ & $\alpha_{c}$ & $\mathrm{f}$ & Error \\
\hline $0-1$ & 0.71 & 14 & 5.937 & - & - & - \\
$1-2$ & 1.58 & 147 & 5.937 & - & - & - \\
$2-3$ & 2.55 & 388 & 5.937 & - & - & - \\
$3-4$ & 3.54 & 594 & 5.938 & - & - & - \\
$4-5$ & 4.53 & 757 & 5.938 & 3.090 & 76.779 & 3.006 \\
$5-6$ & 5.52 & 859 & 5.939 & 2.017 & 44.195 & 1.711 \\
$6-7$ & 6.52 & 852 & 5.940 & 1.679 & 29.077 & 1.200 \\
$7-8$ & 7.52 & 786 & 5.940 & 1.443 & 18.120 & 0.858 \\
$8-9$ & 8.51 & 770 & 5.942 & 1.386 & 14.044 & 0.720 \\
$9-10$ & 9.51 & 753 & 5.943 & 1.305 & 10.516 & 0.600 \\
$10-12$ & 11.05 & 1379 & 5.945 & 1.240 & 6.422 & 0.333 \\
$12-14$ & 13.04 & 1313 & 5.948 & 1.199 & 3.692 & 0.266 \\
$14-16$ & 15.03 & 1336 & 5.952 & 1.180 & 2.408 & 0.229 \\
$16-18$ & 17.03 & 1425 & 5.956 & 1.170 & 1.845 & 0.207 \\
$18-20$ & 19.03 & 1397 & 5.961 & 1.152 & 0.779 & 0.180 \\
$20-25$ & 22.64 & 4125 & 5.971 & 1.153 & 0.758 & 0.105 \\
$25-30$ & 27.61 & 4793 & 5.988 & 1.145 & 0.365 & 0.092 \\
$30-35$ & 32.60 & 5393 & 6.008 & 1.138 & 0.005 & 0.082 \\
$35-40$ & 37.58 & 6351 & 6.031 & 1.142 & 0.124 & 0.077 \\
$40-50$ & 45.28 & 14599 & 6.071 & 1.138 & -0.196 & 0.049 \\
$50-60$ & 55.23 & 18438 & 6.135 & 1.141 & -0.044 & 0.045 \\
$60-70$ & 65.19 & 22059 & 6.207 & 1.143 & -0.036 & 0.042 \\
$70-80$ & 75.17 & 25859 & 6.289 & 1.147 & 0.004 & 0.039 \\
$80-90$ & 85.15 & 29678 & 6.376 & 1.148 & 0.005 & 0.037 \\
$90-100$ & 95.13 & 33793 & 6.468 & 1.151 & 0.046 & 0.035 \\
& \multicolumn{7}{c}{ Sectors used: } & $1-8$ & & \\
& \multicolumn{7}{c}{ Surface Fit Order: } & $2 \times 2$ & & \\
\hline \hline & & Image Area A: & 24.1 & arcsec & & \\
\hline
\end{tabular}


Table 9. NGC 4590 Star Counts.

\begin{tabular}{|c|c|c|c|c|c|c|}
\hline$r$ & $r_{e f f}$ & $\mathrm{~N}$ & $\mathrm{f}_{b k d}$ & $\alpha_{c}$ & $\mathrm{f}$ & Error \\
\hline $0-1$ & 0.71 & 2 & 0.263 & - & - & - \\
\hline $1-2$ & 1.58 & 82 & 0.263 & - & - & - \\
\hline $2-3$ & 2.55 & 232 & 0.263 & 2.261 & 33.131 & 2.192 \\
\hline $3-4$ & 3.54 & 272 & 0.263 & 1.524 & 18.584 & 1.143 \\
\hline $4-5$ & 4.53 & 250 & 0.263 & 1.286 & 11.111 & 0.719 \\
\hline $5-6$ & 5.52 & 239 & 0.263 & 1.192 & 7.984 & 0.533 \\
\hline $6-7$ & 6.52 & 167 & 0.263 & 1.121 & 1.322 & 0.355 \\
\hline $7-8$ & 7.52 & 130 & 0 & 1.092 & 2.750 & 0.264 \\
\hline $8-9$ & 8.51 & 118 & 0.263 & 1 & 01 & 0.218 \\
\hline $9-10$ & 9. & 88 & 0 & 4 & 66 & 0.167 \\
\hline $10-12$ & 11. & 144 & 0.2 & 1 & 0.832 & 0.091 \\
\hline $12-14$ & 1 & 127 & 0.263 & 1 & 0.547 & 0.072 \\
\hline $14-16$ & 15 & 104 & 0.263 & 1 & 0.310 & 0.056 \\
\hline $16-18$ & 17.03 & 85 & 0.263 & 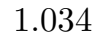 & 0.149 & 0.045 \\
\hline $18-20$ & 19.03 & 90 & 0.263 & 1.0 & 0.127 & 0.041 \\
\hline $20-25$ & 22.64 & 196 & 0.263 & 1.0 & 0.024 & 0.020 \\
\hline $25-30$ & 27.61 & 245 & 0.263 & 1.0 & 0.030 & 0.019 \\
\hline $30-35$ & 32.60 & 261 & 0.263 & 1.032 & 0.001 & 0.016 \\
\hline $35-40$ & 37.58 & 348 & 0.263 & 1.032 & 0.042 & 0.016 \\
\hline $40-50$ & 45.28 & 694 & 0.263 & 1.031 & -0.010 & 0.010 \\
\hline $50-60$ & 55.23 & 886 & 0.263 & 1.033 & 0.002 & 0.009 \\
\hline $60-70$ & 65.19 & 1050 & 0.263 & 1.032 & 0.003 & 0.008 \\
\hline $70-80$ & 75.17 & 1121 & 0.263 & 1.032 & -0.017 & 0.007 \\
\hline $80-$ & 85.15 & 1385 & 0.263 & 1.033 & 0.005 & 0.007 \\
\hline $90-100$ & 95.13 & 1546 & 0.263 & 1.033 & 0.005 & 0.007 \\
\hline \multicolumn{4}{|c|}{ Sectors used: } & \multicolumn{3}{|l|}{$1-8$} \\
\hline & \multicolumn{3}{|c|}{ Surface Fit Order: } & \multicolumn{3}{|l|}{$1 \times 1$} \\
\hline & \multicolumn{3}{|c|}{ Image Area $A$ : } & \multicolumn{3}{|c|}{$28.9 \operatorname{arcsec}^{2}$} \\
\hline
\end{tabular}


TABLe 10. NGC 5824 Star Counts.

\begin{tabular}{|c|c|c|c|c|c|c|}
\hline$r$ & $r_{e f f}$ & $\mathrm{~N}$ & $\mathrm{f}_{b k d}$ & $\alpha_{c}$ & $\mathrm{f}$ & Error \\
\hline $0-1$ & 0.71 & 23 & 2.719 & - & - & - \\
\hline $1-2$ & 1.58 & 158 & 2.719 & 2.551 & 40.049 & 3.402 \\
\hline $2-3$ & 2.55 & 172 & 2.719 & 1.412 & 12.740 & 1.179 \\
\hline $3-4$ & 3.54 & 147 & 2.719 & 1.213 & 5.387 & 0.669 \\
\hline $4-5$ & 4.53 & 132 & 2.719 & 1.152 & 2.659 & 0.468 \\
\hline $5-6$ & 5.52 & 126 & 2.719 & 1.145 & 1.457 & 0.372 \\
\hline $6-7$ & 6.52 & 145 & 2.719 & 1.145 & 1.347 & 0.338 \\
\hline $7-8$ & 7.52 & 170 & 2.719 & 1.140 & 1.394 & 0.315 \\
\hline $8-9$ & 8.51 & 155 & 2.719 & 1.123 & 0.542 & 0.262 \\
\hline $9-10$ & 9.51 & 162 & 2.719 & 1.109 & 0.291 & 0.236 \\
\hline $10-12$ & 11.05 & 394 & 2.719 & 1.114 & 0.456 & 0.160 \\
\hline $12-14$ & 13.04 & 433 & 2.719 & 1.115 & 0.238 & 0.142 \\
\hline $14-16$ & 15.03 & 503 & 2.719 & 1.114 & 0.255 & 0.133 \\
\hline $16-18$ & 17.03 & 564 & 2.719 & 1.111 & 0.216 & 0.124 \\
\hline $18-20$ & 19.03 & 626 & 2.719 & 1.107 & 0.185 & 0.116 \\
\hline $20-25$ & 22.64 & 1762 & 2.719 & 1.107 & 0.041 & 0.066 \\
\hline $25-30$ & 27.61 & 2166 & 2.719 & 1.105 & 0.052 & 0.060 \\
\hline $30-35$ & 32.60 & 2533 & 2.719 & 1.107 & 0.028 & 0.055 \\
\hline $35-40$ & 37.58 & 2954 & 2.719 & 1.111 & 0.067 & 0.051 \\
\hline $40-50$ & 45.28 & 7041 & 2.719 & 1.109 & 0.043 & 0.033 \\
\hline $50-60$ & 55.23 & 8440 & 2.719 & 1.110 & -0.008 & 0.030 \\
\hline $60-70$ & 65.19 & 10153 & 2.719 & 1.111 & 0.043 & 0.027 \\
\hline $70-80$ & 75.17 & 11568 & 2.719 & 1.109 & 0.003 & 0.025 \\
\hline $80-90$ & 85.15 & 13211 & 2.719 & 1.109 & 0.026 & 0.024 \\
\hline $90-100$ & 95.13 & 14536 & 2.719 & 1.109 & -0.017 & 0.022 \\
\hline & \multirow{3}{*}{\multicolumn{3}{|c|}{$\begin{array}{r}\text { Sectors used: } \\
\text { Surface Fit Order: } \\
\text { Image Area } A \text { : }\end{array}$}} & \multicolumn{3}{|l|}{$1-8$} \\
\hline & & & & \multicolumn{3}{|l|}{$1 \times 1$} \\
\hline & & & & \multicolumn{3}{|c|}{$27.2 \operatorname{arcsec}^{2}$} \\
\hline
\end{tabular}


TABLe 11. NGC 6864 Star Counts.

\begin{tabular}{crrrrrr}
\hline \hline$r$ & \multicolumn{1}{c}{$r_{\text {eff }}$} & \multicolumn{1}{c}{$\mathrm{N}$} & \multicolumn{1}{c}{$\mathrm{f}_{b k d}$} & \multicolumn{1}{c}{$\alpha_{c}$} & \multicolumn{1}{c}{$\mathrm{f}$} & Error \\
\hline $0-1$ & 0.71 & 8 & 1.307 & - & - & - \\
$1-2$ & 1.58 & 84 & 1.307 & 2.126 & 20.345 & 2.362 \\
$2-3$ & 2.55 & 83 & 1.306 & 1.326 & 6.703 & 0.879 \\
$3-4$ & 3.54 & 58 & 1.306 & 1.148 & 2.154 & 0.454 \\
$4-5$ & 4.53 & 48 & 1.306 & 1.118 & 0.863 & 0.313 \\
$5-6$ & 5.52 & 44 & 1.305 & 1.088 & 0.277 & 0.239 \\
$6-7$ & 6.52 & 56 & 1.305 & 1.095 & 0.412 & 0.229 \\
$7-8$ & 7.52 & 53 & 1.304 & 1.087 & 0.093 & 0.192 \\
$8-9$ & 8.51 & 62 & 1.304 & 1.091 & 0.144 & 0.184 \\
$9-10$ & 9.51 & 54 & 1.304 & 1.080 & -0.187 & 0.152 \\
$10-12$ & 11.05 & 162 & 1.303 & 1.098 & 0.168 & 0.116 \\
$12-14$ & 13.04 & 207 & 1.302 & 1.091 & 0.277 & 0.110 \\
$14-16$ & 15.03 & 215 & 1.301 & 1.089 & 0.118 & 0.097 \\
$16-18$ & 17.03 & 220 & 1.301 & 1.091 & -0.016 & 0.087 \\
$18-20$ & 19.03 & 266 & 1.300 & 1.094 & 0.093 & 0.085 \\
$20-25$ & 22.64 & 747 & 1.298 & 1.088 & 0.015 & 0.048 \\
$25-30$ & 27.61 & 955 & 1.297 & 1.092 & 0.084 & 0.045 \\
$30-35$ & 32.60 & 1078 & 1.295 & 1.088 & 0.018 & 0.040 \\
$35-40$ & 37.58 & 1257 & 1.293 & 1.089 & 0.036 & 0.037 \\
$40-50$ & 45.28 & 3016 & 1.290 & 1.088 & 0.036 & 0.024 \\
$50-60$ & 55.23 & 3608 & 1.286 & 1.089 & 0.013 & 0.022 \\
$60-70$ & 65.19 & 4243 & 1.282 & 1.087 & 0.009 & 0.020 \\
$70-80$ & 75.17 & 4924 & 1.278 & 1.090 & 0.023 & 0.019 \\
$80-90$ & 85.15 & 5432 & 1.274 & 1.089 & -0.008 & 0.017 \\
$90-100$ & 95.13 & 6064 & 1.270 & 1.090 & -0.005 & 0.016 \\
& \multicolumn{7}{c}{ Sectors used: } & $1-3,7-8$ & & \\
& \multicolumn{7}{c}{ Surface Fit Order: } & $1 \times 1$ & & \\
\hline \hline & \multicolumn{1}{c}{ Image Area A: } & 37.8 arcsec ${ }^{2}$ & \\
\hline
\end{tabular}


TABLe 12. NGC 6934 Star Counts.

\begin{tabular}{|c|c|c|c|c|c|c|}
\hline$r$ & $r_{e f f}$ & $\mathrm{~N}$ & $\mathrm{f}_{b k d}$ & $\alpha_{c}$ & $\mathrm{f}$ & Error \\
\hline $0-1$ & 0.71 & 4 & 1.019 & - & 一 & 一 \\
\hline $1-2$ & 1.58 & 65 & 1.019 & - & - & - \\
\hline $2-3$ & 2.55 & 148 & 1.019 & 2.076 & 18.537 & 1.608 \\
\hline $3-4$ & 3.54 & 111 & 1.019 & 1.409 & 6.091 & 0.675 \\
\hline $4-5$ & 4.53 & 85 & 1.019 & 1.240 & 2.709 & 0.404 \\
\hline $5-6$ & 5.52 & 53 & 1.019 & 1.195 & 0.813 & 0.252 \\
\hline $6-7$ & 6.52 & 57 & 1.019 & 1.199 & 0.655 & 0.222 \\
\hline $7-8$ & 7.52 & 65 & 1.019 & 9 & 0.621 & 0.203 \\
\hline $8-9$ & 8.51 & 59 & 1 & 5 & 0.257 & 0.166 \\
\hline $9-10$ & 9 . & 52 & 1 & 4 & -0.014 & 0.139 \\
\hline $10-12$ & 11.05 & 123 & 1 & 2 & 0.023 & 0.094 \\
\hline $12-14$ & 13.0 & 164 & 1 & 9 & 0.164 & 0.092 \\
\hline $14-16$ & 15.03 & 181 & 1. & 8 & 0.103 & 0.083 \\
\hline $16-18$ & 17.03 & 207 & $1 .($ & 8 & 0.122 & 0.079 \\
\hline $18-20$ & 19.03 & 183 & 1.019 & 1.165 & -0.126 & 0.066 \\
\hline $20-25$ & 22.64 & 635 & 1.019 & 1.1 & 0.032 & 0.042 \\
\hline $25-30$ & 27.61 & 786 & 1.019 & 1.173 & 0.048 & 0.038 \\
\hline $30-35$ & 32.60 & 861 & 1.019 & 1.167 & -0.035 & 0.034 \\
\hline $35-40$ & 37.58 & 1023 & 1.019 & 1.170 & -0.004 & 0.032 \\
\hline $40-50$ & 45.28 & 2510 & 1.019 & 1.169 & 0.018 & 0.021 \\
\hline $50-60$ & 55.23 & 3208 & 1.019 & 1.170 & 0.067 & 0.019 \\
\hline $60-70$ & 65.19 & 3680 & 1.019 & 1.168 & 0.033 & 0.017 \\
\hline $70-80$ & 75.17 & 4066 & 1.019 & 1.166 & -0.013 & 0.016 \\
\hline $80-$ & 85.15 & 4455 & 1.019 & 1.168 & -0.045 & 0.015 \\
\hline $90-100$ & 95.13 & 5051 & 1.019 & 1.165 & -0.034 & 0.014 \\
\hline \multicolumn{4}{|c|}{ Sectors used: } & \multicolumn{3}{|l|}{$1-8$} \\
\hline & \multicolumn{3}{|c|}{ Surface Fit Order: } & \multicolumn{3}{|l|}{$1 \times 1$} \\
\hline & \multicolumn{3}{|c|}{ Image Area $A$ : } & \multicolumn{3}{|c|}{$35.8 \operatorname{arcsec}^{2}$} \\
\hline
\end{tabular}


TABLe 13. NGC 6981 Star Counts.

\begin{tabular}{|c|c|c|c|c|c|c|}
\hline$r$ & $r_{e f f}$ & $\mathrm{~N}$ & $\mathrm{f}_{b k d}$ & $\alpha_{c}$ & $\mathrm{f}$ & Error \\
\hline $0-1$ & 0.71 & 4 & 0.782 & - & - & - \\
\hline $1-2$ & 1.58 & 141 & 0.782 & - & - & - \\
\hline $2-3$ & 2.55 & 244 & 0.782 & 1.874 & 28.324 & 1.863 \\
\hline $3-4$ & 3.54 & 158 & 0.782 & 1.220 & 7.980 & 0.697 \\
\hline $4-5$ & 4.53 & 76 & 0.782 & 1.109 & 2.200 & 0.342 \\
\hline $5-6$ & 5.52 & 57 & 0.782 & 1.076 & 0.993 & 0.235 \\
\hline $6-7$ & 6.52 & 59 & 0.782 & 1.079 & 0.776 & 0.203 \\
\hline $7-8$ & 7.52 & 46 & 0.782 & 1.064 & 0.257 & 0.153 \\
\hline $8-9$ & 8. & 53 & 0.782 & 73 & 0.283 & 0.146 \\
\hline $9-10$ & 9. & 43 & 82 & 4 & -0.016 & 0.117 \\
\hline $10-12$ & 11 & 105 & 82 & 0 & 0.023 & 0.079 \\
\hline $12-14$ & 1 & 107 & 0.782 & 3 & -0.086 & 0.067 \\
\hline $14-16$ & 1 & 142 & 0.782 & 1 & 0.021 & 0.067 \\
\hline $16-18$ & 17.03 & 145 & 0.782 & 1.0 & -0.060 & 0.060 \\
\hline $18-20$ & 19.03 & 173 & 0.782 & 1.064 & -0.011 & 0.059 \\
\hline $20-25$ & 22.64 & 499 & 0.782 & 1.064 & -0.031 & 0.034 \\
\hline $25-30$ & 27.61 & 617 & 0.782 & 1.063 & -0.023 & 0.031 \\
\hline $30-35$ & 32.60 & 703 & 0.782 & 1.063 & -0.050 & 0.028 \\
\hline $35-40$ & 37.58 & 875 & 0.782 & 1.064 & 0.008 & 0.027 \\
\hline $40-50$ & 45.28 & 2086 & 0.782 & 1.067 & 0.005 & 0.017 \\
\hline $50-60$ & 55.23 & 2589 & 0.782 & 1.068 & 0.018 & 0.016 \\
\hline $60-70$ & 65.19 & 3025 & 0.782 & 1.068 & 0.009 & 0.014 \\
\hline $70-80$ & 75.17 & 3480 & 0.782 & 1.068 & 0.007 & 0.013 \\
\hline 80 & 85.15 & 3983 & 0.782 & 1.069 & 0.015 & 0.013 \\
\hline $90-100$ & 95.13 & 4305 & 0.782 & 1.068 & -0.012 & 0.012 \\
\hline \multicolumn{4}{|c|}{$\begin{array}{l}\text { Sectors used: } \\
\text { S }\end{array}$} & \multicolumn{3}{|l|}{$1-8$} \\
\hline & \multicolumn{3}{|c|}{ Surface Fit Order: } & \multicolumn{3}{|l|}{$1 \times 1$} \\
\hline & \multicolumn{3}{|c|}{ Image Area $A$ : } & \multicolumn{3}{|c|}{$25.7 \operatorname{arcsec}^{2}$} \\
\hline
\end{tabular}


TABLE 14. NGC 7078 Star Counts.

\begin{tabular}{|c|c|c|c|c|c|c|}
\hline$r$ & $r_{e f f}$ & $\mathrm{~N}$ & $\mathrm{f}_{b k d}$ & $\alpha_{c}$ & $\mathrm{f}$ & Error \\
\hline $0-1$ & 0.71 & 0 & 0.236 & - & - & - \\
\hline $1-2$ & 1.58 & 13 & 0.236 & - & - & - \\
\hline $2-3$ & 2.55 & 87 & 0.236 & - & - & - \\
\hline $3-4$ & 3.54 & 248 & 0.236 & - & - & - \\
\hline $4-5$ & 4.53 & 404 & 0.236 & - & - & - \\
\hline $5-6$ & 5.52 & 425 & 0.236 & 2.012 & 24.505 & 1.200 \\
\hline $6-7$ & 6.52 & 378 & 0.236 & 1.563 & 14.232 & 0.744 \\
\hline $7-8$ & 7.52 & 319 & 0.236 & 1.323 & 8.716 & 0.501 \\
\hline $8-9$ & 8.51 & 260 & 0.236 & .230 & .751 & 0.371 \\
\hline $9-10$ & 9.51 & 206 & 0.236 & 197 & 95 & 0.288 \\
\hline $10-12$ & 11.0 & 310 & 0.236 & 129 & .295 & 0.144 \\
\hline $12-14$ & $1:$ & 200 & 0.236 & 097 & 1.106 & 0.095 \\
\hline $14-16$ & 15 & 123 & 0.236 & 1.077 & 0.467 & 0.063 \\
\hline $16-18$ & 17.03 & 89 & 0.236 & 1.075 & 0.211 & 0.047 \\
\hline $18-20$ & 19.03 & 87 & 0.236 & 1.072 & 0.154 & 0.042 \\
\hline $20-25$ & 22.64 & 194 & 0.236 & 1.068 & 0.057 & 0.021 \\
\hline $25-30$ & 27.61 & 204 & 0.236 & 1.067 & 0.015 & 0.018 \\
\hline $30-35$ & 32.60 & 210 & 0.236 & 1.069 & -0.017 & 0.015 \\
\hline $35-40$ & 37.58 & 228 & 0.236 & 1.066 & -0.030 & 0.014 \\
\hline $40-50$ & 45.28 & 588 & 0.236 & 1.067 & -0.015 & 0.009 \\
\hline $50-60$ & 55.23 & 756 & 0.236 & 1.068 & -0.003 & 0.008 \\
\hline $60-70$ & 65.19 & 897 & 0.236 & 1.068 & -0.002 & 0.008 \\
\hline $70-80$ & 75.17 & 995 & 0.236 & 1.067 & -0.011 & 0.007 \\
\hline $80-$ & 85.15 & 1177 & 0.236 & 1.067 & -0.001 & 0.007 \\
\hline $90-100$ & 95.13 & 1368 & 0.236 & 1.068 & 0.008 & 0.007 \\
\hline \multicolumn{4}{|c|}{$\begin{array}{l}\text { Sectors used: } \\
\text { S }\end{array}$} & \multicolumn{3}{|l|}{$1-8$} \\
\hline & \multirow{2}{*}{\multicolumn{3}{|c|}{$\begin{array}{l}\text { Surface Fit Order: } \\
\text { Image Area } A:\end{array}$}} & \multicolumn{3}{|l|}{$1 \times 1$} \\
\hline & & & & \multicolumn{3}{|c|}{$37.8 \operatorname{arcsec}^{2}$} \\
\hline
\end{tabular}


TABLE 15. NGC 7089 Star Counts.

\begin{tabular}{|c|c|c|c|c|c|c|}
\hline$r$ & $r_{e f f}$ & $\mathrm{~N}$ & $\mathrm{f}_{b k d}$ & $\alpha_{c}$ & $\mathrm{f}$ & Error \\
\hline $0-1$ & 0.71 & 0 & 0.306 & - & - & 一 \\
\hline $1-2$ & 1.58 & 16 & 0.306 & - & - & - \\
\hline $2-3$ & 2.55 & 138 & 0.306 & - & - & - \\
\hline $3-4$ & 3.54 & 336 & 0.306 & - & - & - \\
\hline $4-5$ & 4.53 & 484 & 0.306 & - & 一 & - \\
\hline $5-6$ & 5.52 & 441 & 0.306 & 1.960 & 24.702 & 1.191 \\
\hline $6-7$ & 6.52 & 363 & 0.306 & 1.396 & 12.106 & 0.651 \\
\hline $7-8$ & 7.52 & 262 & 0.306 & 1.223 & 6.493 & 0.420 \\
\hline $8-9$ & 8.51 & 165 & 0 & 1.146 & .234 & 0.276 \\
\hline $9-10$ & 9.51 & 114 & 0 & 107 & .809 & 0.198 \\
\hline $10-12$ & 11.05 & 147 & 0. & 1.081 & 0.844 & 0.095 \\
\hline $12-14$ & 13.04 & 113 & 0.3 & 1.066 & 0.431 & 0.069 \\
\hline $14-16$ & 15.03 & 83 & 0.3 & 1.062 & 0.162 & 0.051 \\
\hline $16-18$ & 17.03 & 83 & 0.306 & 1.056 & 0.104 & 0.045 \\
\hline $18-20$ & 19.03 & 96 & 0.306 & 1.061 & 0.120 & 0.044 \\
\hline $20-25$ & 22.64 & 248 & 0.306 & 1.057 & 0.065 & 0.024 \\
\hline $25-30$ & 27.61 & 279 & 0.306 & 1.058 & 0.036 & 0.020 \\
\hline $30-35$ & 32.60 & 317 & 0.306 & 1.059 & 0.023 & 0.018 \\
\hline $35-40$ & 37.58 & 366 & 0.306 & 1.058 & 0.023 & 0.017 \\
\hline $40-50$ & 45.28 & 840 & 0.306 & 1.057 & 0.008 & 0.011 \\
\hline $50-60$ & 55.23 & 986 & 0.306 & 1.058 & -0.004 & 0.010 \\
\hline $60-70$ & 65.19 & 1218 & 0.306 & 1.059 & 0.010 & 0.009 \\
\hline $70-80$ & 75.17 & 1402 & 0.306 & 1.059 & 0.009 & 0.008 \\
\hline $80-?$ & 85.15 & 1520 & 0.306 & 1.058 & -0.005 & 0.008 \\
\hline $90-100$ & 95.13 & 1699 & 0.306 & 1.056 & -0.005 & 0.007 \\
\hline \multicolumn{4}{|c|}{ Sectors used: } & \multicolumn{3}{|l|}{$1-8$} \\
\hline & \multicolumn{3}{|c|}{ Surface Fit Order: } & \multicolumn{3}{|l|}{$1 \times 1$} \\
\hline & \multicolumn{3}{|c|}{ Image Area $A$ : } & \multicolumn{3}{|c|}{$34.0 \operatorname{arcsec}^{2}$} \\
\hline
\end{tabular}


Table 16. Sources of Surface Density Data.

\begin{tabular}{ll}
\hline \hline \multicolumn{1}{c}{ Code } & \multicolumn{1}{c}{ Source } \\
\hline CF & Chun \& Freeman 1979 \\
CJP & Peterson 1986b \\
CP & Peterson 1976 \\
GFIQ & Present Study \\
HAM & Hamuy 1984 \\
HB & Hanes \& Brodie 1985 \\
HLJ & Johnson 1959 \\
HST & Lauer et al. 1991 \\
II & Illingworth \& Illingworth 1976 \\
KHHW & King et al. 1968 \\
KM & Kron \& Mayall 1960 \\
KR & Kron \& Gordon 1986 \\
& \\
\hline \hline
\end{tabular}


TABle 17. Fitted Tidal Radii.

\begin{tabular}{lccccc}
\hline \hline Cluster & $\begin{array}{c}r_{c} \\
(\operatorname{arcmin})\end{array}$ & $\begin{array}{c}r_{t} \\
(\operatorname{arcmin})\end{array}$ & $\begin{array}{c}\text { Fitted Region } \\
(\operatorname{arcmin})\end{array}$ & $\tilde{\chi}^{2}$ & $\begin{array}{c}90 \% \text { Confidence Interval } \\
(\operatorname{arcmin})\end{array}$ \\
\hline NGC 288 & 1.40 & 17.4 & $4-12$ & 0.43 & $16.7-17.8$ \\
NGC 362 & 0.20 & 14.2 & $4-10$ & 4.32 & $13.9-14.5$ \\
NGC 1904 & 0.23 & 11.0 & $2-7$ & 1.80 & $10.8-11.7$ \\
NGC 2808 & 0.28 & 14.5 & $4-15$ & 1.20 & $14.0-15.0$ \\
NGC 3201 & 1.10 & 34.7 & $4-20$ & 3.08 & $33.2-36.2$ \\
NGC 4590 & 0.80 & 25.2 & $2-12$ & 1.31 & $23.2-27.0$ \\
NGC 5824 & 0.01 & 11.6 & $1-6$ & 0.66 & $10.4-13.4$ \\
NGC 6864 & 0.11 & 9.3 & $1-6$ & 0.08 & $7.7-10.9$ \\
NGC 6934 & 0.22 & 9.7 & $2-6$ & 0.48 & $8.6-10.7$ \\
NGC 6981 & 0.42 & 8.3 & $2-6$ & 1.68 & $7.6-8.6$ \\
NGC 7078 & 0.22 & 23.2 & $5-16$ & 1.92 & $22.8-23.7$ \\
NGC 7089 & 0.37 & 15.9 & $5-12$ & 2.88 & $15.7-16.3$ \\
& & & & \\
\hline \hline
\end{tabular}

